\title{
An experimental investigation on the effects of freestream turbulence intensity on film cooling effectiveness and heat transfer coefficient for an anti-vortex hole
}

\author{
Stephen Andrew Hayes
}

Follow this and additional works at: https://researchrepository.wvu.edu/etd

\section{Recommended Citation \\ Hayes, Stephen Andrew, "An experimental investigation on the effects of freestream turbulence intensity on film cooling effectiveness and heat transfer coefficient for an anti-vortex hole" (2014). Graduate Theses, Dissertations, and Problem Reports. 5783. \\ https://researchrepository.wvu.edu/etd/5783}

This Thesis is protected by copyright and/or related rights. It has been brought to you by the The Research Repository @ WVU with permission from the rights-holder(s). You are free to use this Thesis in any way that is permitted by the copyright and related rights legislation that applies to your use. For other uses you must obtain permission from the rights-holder(s) directly, unless additional rights are indicated by a Creative Commons license in the record and/ or on the work itself. This Thesis has been accepted for inclusion in WVU Graduate Theses, Dissertations, and Problem Reports collection by an authorized administrator of The Research Repository @ WVU. For more information, please contact researchrepository@mail.wvu.edu. 


\title{
AN EXPERIMENTAL INVESTIGATION ON THE EFFECTS OF FREESTREAM TURBULENCE INTENSITY ON FILM COOLING EFFECTIVENESS AND HEAT TRANSFER COEFFICIENT FOR AN ANTI-VORTEX HOLE
}

\author{
Stephen Andrew Hayes \\ Thesis submitted \\ to the Benjamin M. Statler College of Engineering and Mineral Resources \\ at West Virginia University \\ in partial fulfillment of the requirements for the degree of \\ Masters of Science in \\ Mechanical Engineering
}

Andrew C. Nix, Ph.D., Chair

John M. Kuhlman, Ph.D.

Wade W. Huebsch, Ph.D.

Department of Mechanical and Aerospace Engineering

Morgantown, West Virginia 2014

Keywords: Gas Turbines, Film Cooling, Heat Transfer, Infrared Thermography, AntiVortex Hole

Copyright 2014 Stephen A. Hayes 


\begin{abstract}
AN EXPERIMENTAL INVESTIGATION ON THE EFFECTS OF FREESTREAM TURBULENCE INTENSITY ON FILM COOLING EFFECTIVENESS AND HEAT TRANSFER COEFFICIENT FOR AN ANTI-VORTEX HOLE
\end{abstract}

\title{
Stephen Andrew Hayes
}

Film cooling is used to thermally protect combustor and turbine components by creating a layer of relatively cooler air than the freestream air to insulate the components from the hot freestream gases. This relatively cooler air is taken from upstream in the high-pressure compressor section at a loss to the engine efficiency, and therefore must be used as effectively as possible. The efficiency gained from increasing the turbine inlet temperature outweighs the loss due to extracting air from the compressor section if the cooling air is used effectively. A novel anti-vortex hole (AVH) geometry has been investigated experimentally through a transient infrared thermography technique to study the film cooling effectiveness and surface convective heat transfer coefficients for varying blowing ratio and freestream turbulence intensity. A major concern with the AVH will be how the secondary jets counteract the main counter rotating vortex (CRV) pair at increased freestream turbulence levels. This is the first experimental facility to study the effects of higher freestream turbulence levels on an AVH geometry. Furthermore, this is the first experimental investigation to report centerline film cooling effectiveness and the convective heat transfer coefficient that had not been reported in prior studies. The AVH geometry is designed with two secondary holes stemming from a main cooling hole; these holes attempt to diffuse the coolant jet and mitigate the vorticity produced by conventional straight holes. This geometry shows improved results at low turbulence intensities compared to conventional straight holes. Three freestream turbulence intensities of $1,7.5$, and $11.7 \%$ were investigated at blowing ratios of $0.5,1.0,1.5$, and 2.0 to form a test matrix of twelve different test conditions. Results showed that the higher freestream turbulence conditions were beneficial in the performance of the AVH. Increasing the blowing ratio at all turbulence levels also improved film cooling effectiveness both span-averaged and on the centerline. The highest performing case was at a turbulence intensity of $7.5 \%$ and a blowing ratio of 2.0 . The $11.7 \%$ cases outperformed the $1 \%$ cases, but it appears that at $11.7 \%$ cases that the higher freestream turbulence reduces the performance of the secondary holes compared to the $7.5 \%$ cases. Increasing the blowing ratio and turbulence intensity will result in a higher heat transfer coefficient, and thus must be taken into account for future designs. 


\section{DEDICATION}

I dedicate this thesis to my cousin Ruby Nicole Anderson. Although we did not get to meet in this life, I look forward to meeting you in the next.

Love,

Steve

"Your tiny footprints embedded in our hearts."

Unknown 


\section{ACKNOWLEDGEMENTS}

"Whether you think you can or you can't, you're right."

I would like to start by thanking my family members. My parents, Gary and Myrna Hayes, without the values that they have instilled in me from a young age and their unwavering support, this would not have been possible. Julie Eliasen and Kevin Hayes, I want to thank them for lifting my spirits during some of my late nights working.

I would like to thank my advisor Dr. Nix. I sincerely appreciate him bringing me on as a graduate research assistant during my time here. I was able to learn much in the courses he taught me and he was never afraid to encourage me to reach for higher goals. Our friendship is one that I am fortunate and grateful to have.

I was fortunate enough to have Dr. Kuhlman for three classes throughout my time at WVU. I never thought it was possible to cover so much material in a 50 minute class. Each class was a marathon and a sprint to take notes in (my hand started to cramp typing this), but were each enjoyable and informative.

Dr. Huebsch taught my undergraduate Fluid Mechanics class and was always willing to help students outside of office hours through exam review sessions and homework help, which made a difference and shows his willingness to help students. I would like to thank Dr. Huebsch and Dr. Kuhlman for being on my committee

I would like to thank my good friend and fellow graduate student Tim Repko. He introduced me to Dr. Nix and without him I would not have known about this opportunity. He was also very helpful in explaining concepts and fundamentals to me as I got up to speed in the beginning of my graduate work.

I would also like to thank the undergraduate students that helped me throughout my research. Dustin Frohnapfel, Evan Ford, and Chad Jones, I cannot thank them enough for the help that they provided me with designing and constructing the facility as well as creating early iterations of data collection codes. We were never past giving each other a hard time in the office and it kept the atmosphere light. I would like to thank Josh Everett and Daniel Whitlow for their help with collecting data and assistance in characterizing the 
facility. Thanks to Dave Billups for his help with the labor intensive post processing of the data collected during the transient tests and for editing my thesis.

I would also like to thank graduate student Nathan Weese for his help designing the coolant loop of the facility and for his help in troubleshooting the facility. I would like to thank graduate student Kevin Luo for his help post processing data when he didn't have to help at all. I would like to thank my friends in the ESB Annex 280 office: Josh Matheny, Cesar Sandoval, Kevin Luo, and John Hailer for always being there to joke around and for reminding me that taking a night off is just as necessary as another late night in the office.

Cliff Judy in the Mechanical and Aerospace Engineering Machine Shop was an immense help in teaching me how to use machinery in the shop as well as machining a few components of the facility himself. His advice and tips helped save time and money during the fabrication stage. I would like to thank Richard Atkinson, Bradley Ralston, Wayne Hildebrand, Daniel Carder, Zac Luzader, and Josh Israel as well as other members of CAFEE. They were always willing to help me if problems occurred while I was down in the ERC and helped troubleshoot equipment that was not functioning properly. I would also like to thank Dr. Jay Wilhelm for some of his time helping with any computer related questions I had. I would like to thank my friends that read through my initial thesis rough draft as their input was valuable. Finally, I would like to thank my friends and family that supported me these past two years and were understanding when I wasn't able to see or spend time with them. 


\section{CONTENTS}

ABSTRACT

1.1 BACKGROUND ON GAS TURBINE ENGINES ................................................... 1

1.1.1 EARLY HISTORY OF GAS TURBINE ENGINES .............................................. 1

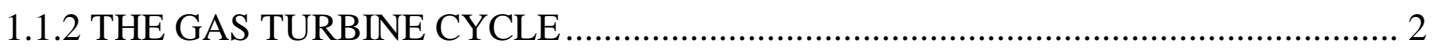

1.2 HEAT TRANSFER AND COOLING IN GAS TURBINE ENGINES ............................. 6

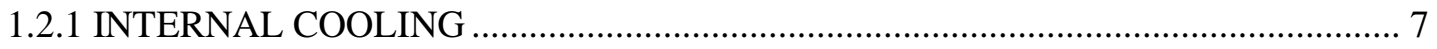

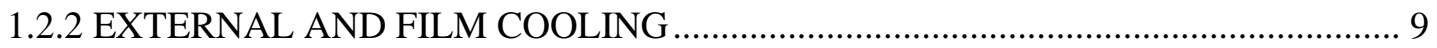

1.3 MEASURING TECHNIQUES OF FILM COOLING .................................................... 9

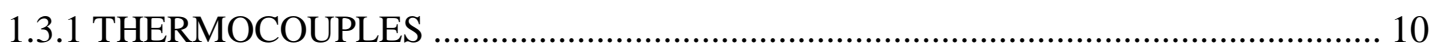

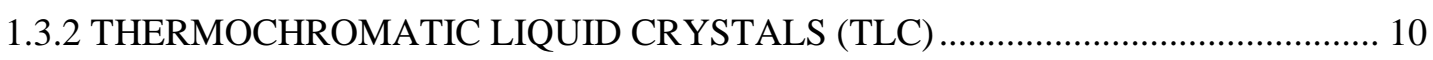

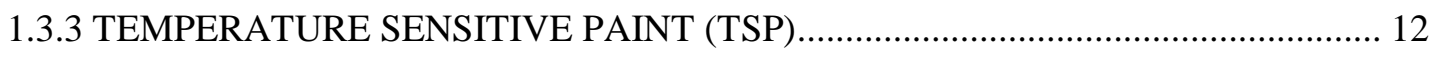

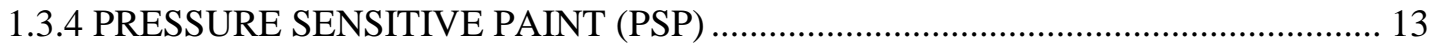

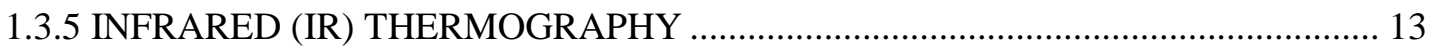

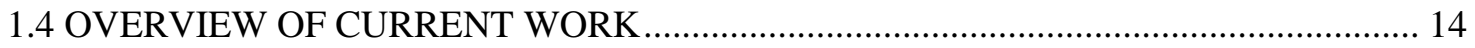

1.4.1 DESIGN, FABRICATION, AND BENCHMARKING OF NEW FACILITY ........... 14

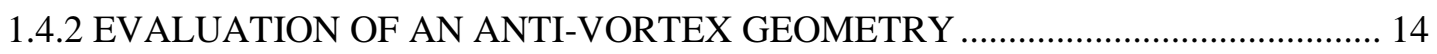

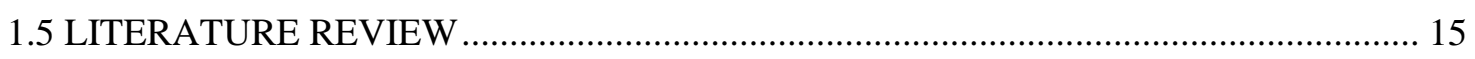

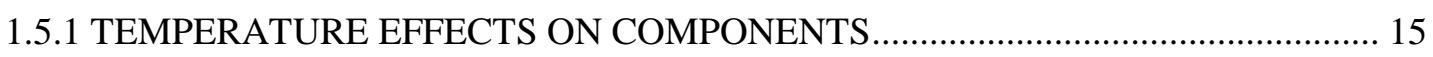

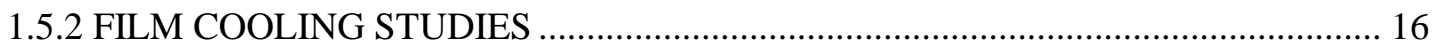

1.5.3 CONVENTIONAL STRAIGHT HOLE STUDIES ................................................. 17

1.5.4 SHAPED AND ADVANCED COOLING HOLE GEOMETRIES ............................ 19

1.5.5 ANTI-VORTEX COOLING HOLE GEOMETRY ….................................................. 24

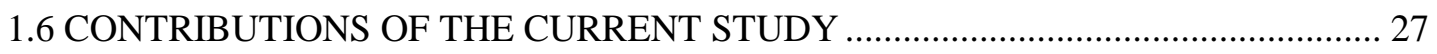

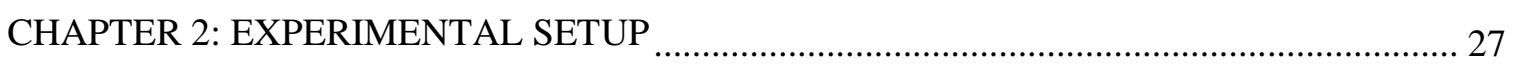


2.1 DESIGN, FABRICATION, AND LAYOUT OF EXPERIMENTAL FACILITY. 27

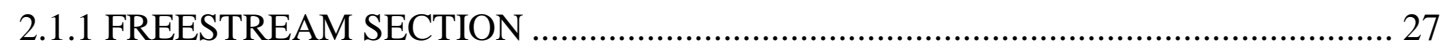

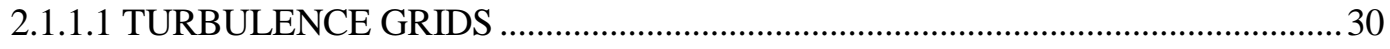

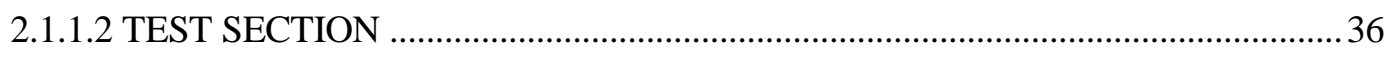

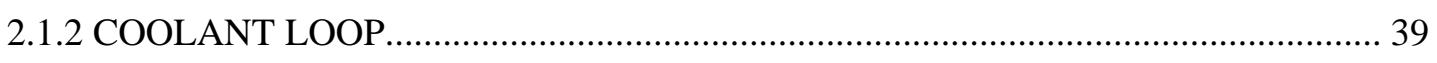

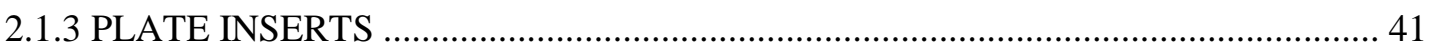

2.2 DATA ACQUISITION (DAQ) AND INSTRUMENTATION ....................................... 43

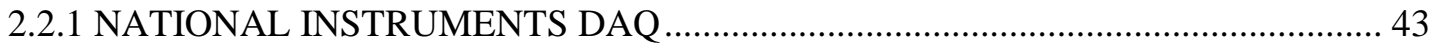

2.2.2 HOTWIRE ANEMOMETER AND PITOT-STATIC PROBE ................................. 44

2.2.3 THERMOCOUPLES AND PRESSURE TRANSDUCERS ........................................ 46

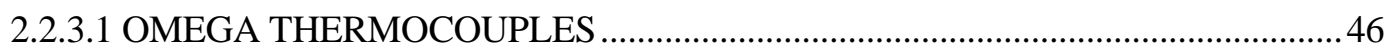

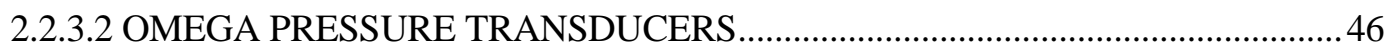

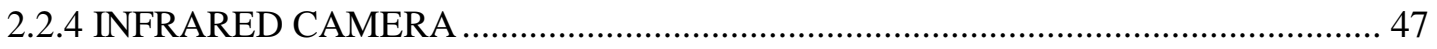

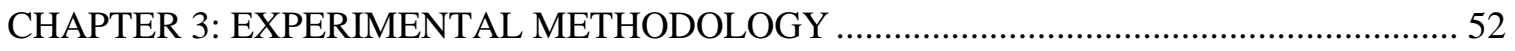

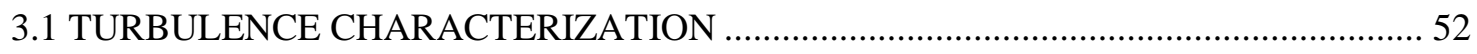

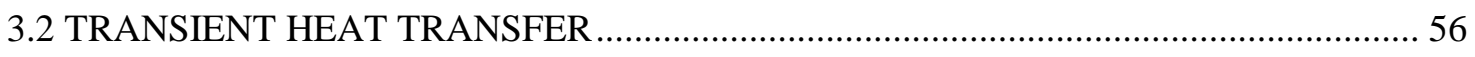

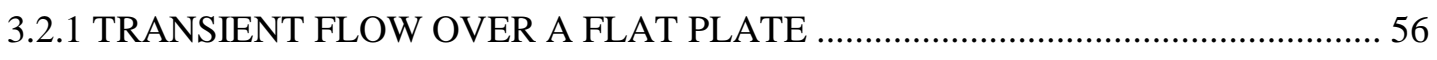

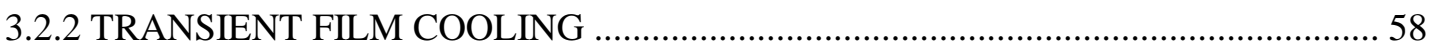

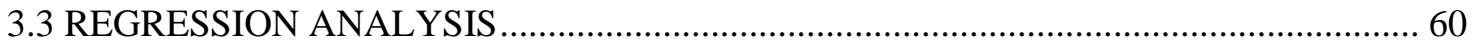

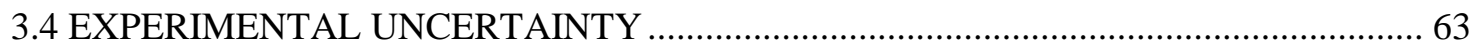

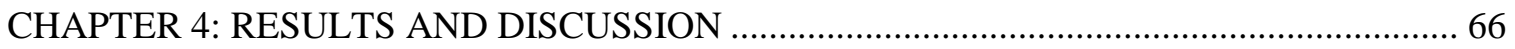

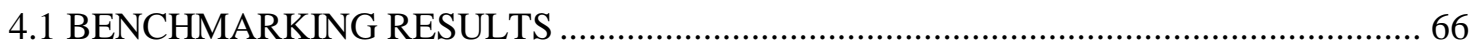

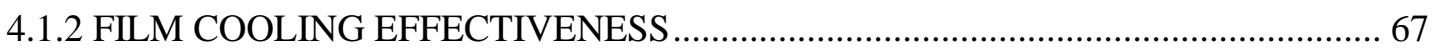

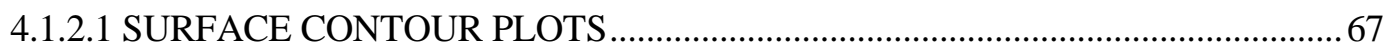

4.1.2.2 SPAN-AVERAGED FILM COOLING EFFECTTIVENESS ...................................6

4.1.2.3 CENTERLINE FILM COOLING EFFECTIVENESS ......................................... 71

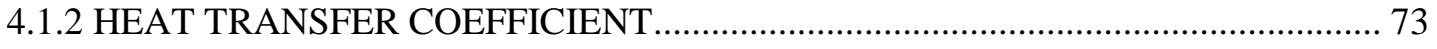

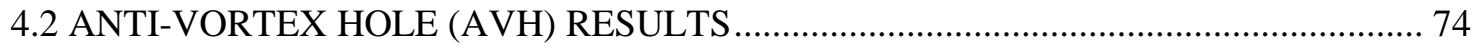

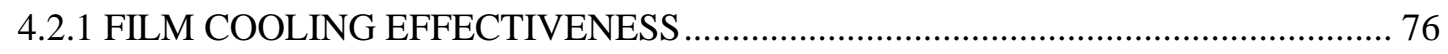

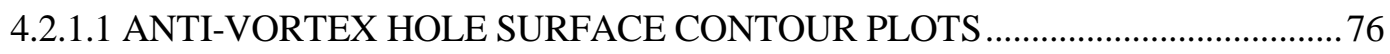

4.2.1.2 ANTI-VORTEX HOLE SPAN-AVERAGED FILM COOLING EFFECTIVENESS78 4.2.1.3 ANTI-VORTEX HOLE CENTERLINE FILM COOLING EFFECTTIVENESS . 81 4.2.1.4 ANTI-VORTEX HOLE AREA-AVERAGED FILM COOLING EFFECTIVENESS 


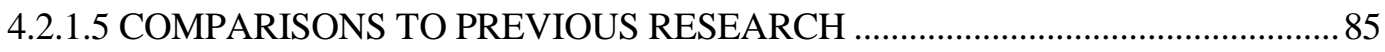

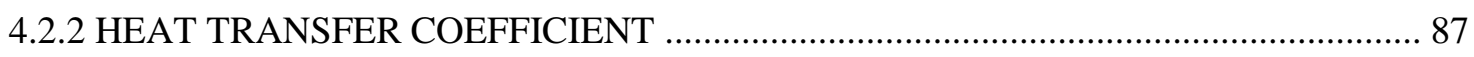

4.2.2.1 ANTI-VORTEX HOLE SPAN-AVERAGED HEAT TRANSFER COEFFICIENT

.88

4.2.2.2 ANTI-VORTEX HOLE CENTERLINE HEAT TRANSFER COEFFICIENT .....93

4.2.2.3 ANTI-VORTEX HOLE AREA-AVERAGED HEAT TRANSFER COEFFICIENT

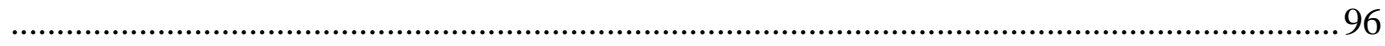

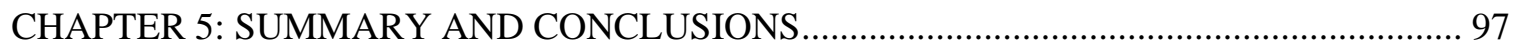

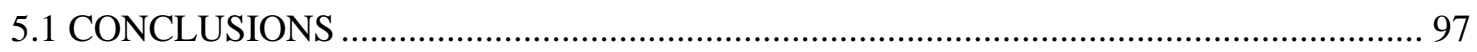

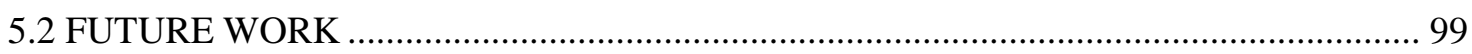

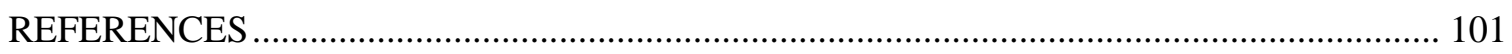

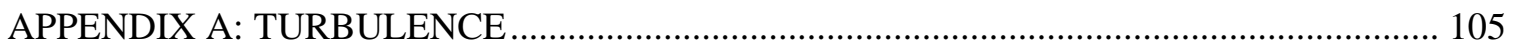




\section{LIST OF TABLES}

Table 1: Experimentally Investigated AVH Geometry Parameters [13] ............................26

Table 2: Straight Hole Benchmark Matrix ...........................................................................66

Table 3: Geometric Parameters of Current AVH Geometry ...............................................75

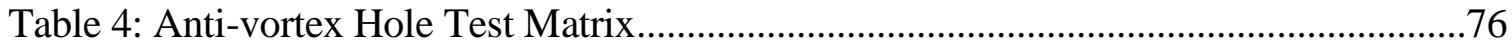

Table 5: Area-averaged Film Cooling Effectiveness .......................................................85

Table 6: Current Study and Previous Numerical Results ..................................................86

Table 7: Area-averaged Dimensionless Heat Transfer Coefficient ......................................96 


\section{LIST OF FIGURES}

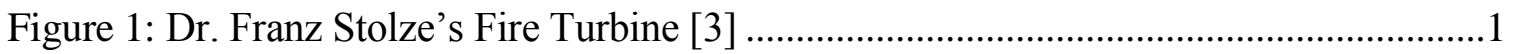

Figure 2: T-s and p-V Diagrams of Ideal Brayton Cycle [6]................................................

Figure 3: Schematics of Propulsion Systems [4] .................................................................

Figure 4: Diagram of Propulsion Systems [7]..................................................................

Figure 5: Turbofan Engine Showing Temperature Gradient of Air through Core [8] ...........6

Figure 6: Gas Turbine Cooling Techniques [9] …………………………………..........

Figure 7: Electromagnetic Spectrum with Emphasized Visible and Infrared Bands [10]....12

Figure 8: Rise in Turbine Inlet Temperature throughout History [17] .................................16

Figure 9: Counter-rotating Vortex Pair and Jet Lift off from Haven et al. [21].....................18

Figure 10: Four Types of Shaped Film Cooling Holes [27] ..............................................21

Figure 11: Four Trench Configurations with Conventional Straight Hole [32] ...................23

Figure 12: Three View Drawing of Anti-Vortex Hole [13] ................................................25

Figure 13: Experimentally Investigated AVH Geometry Configurations [13]......................26

Figure 14: WVU Open Loop Tunnel Schematic ……………………………………….....27

Figure 15: SolidWorks Model of Wind Tunnel Freestream Loop .......................................28

Figure 16: Wind Tunnel Facility during Probe Traverse........................................................29

Figure 17: Freestream Flow through Turbulence Grid [39] .................................................30

Figure 18: Non-Dimensionalized Theoretical Turbulence Intensity and Length Scale for

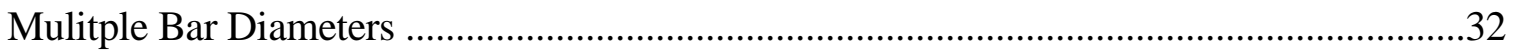

Figure 19: 1/4" and 1/2" Turbulence Grids ......................................................................3

Figure 20: Turbulence Intensity Decay for $1 / 4$ " Grid ..........................................................

Figure 21: Growth of Non-Dimensionalized Turbulence Length Scale for 1/4" Grid ..........34

Figure 22: Turbulence Intensity Decay for $1 / 2$ " Grid ...........................................................

Figure 23: Growth of Non-Dimensionalized Turbulence Length Scale for 1/2" Grid ..........36

Figure 24: First Stage Gas Turbine Vane with Test Section Region of Interest....................36

Figure 25: Test Section with Boundary Layer Suction System................................................37

Figure 26: Test Section Base Shown with Interchangeable Plate Insert.................................38

Figure 27: Traverse System (Left), Pitot-Static and Hotwire Probes (Right) .........................39

Figure 28: Laminar Flow Element [42] ...........................................................................40

Figure 29: Plenum for Coolant Flow ...............................................................................41 
Figure 30: Isometric Views of $30^{\circ}$ Conventional Straight Hole and Selected AVH

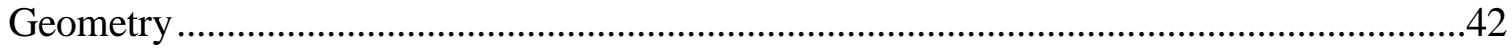

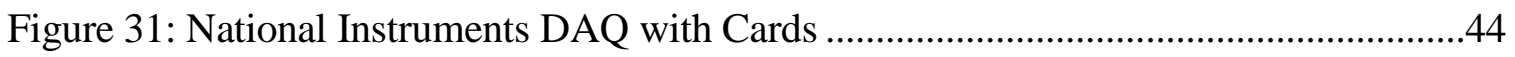

Figure 32: Dantec Dynamics Hotwire Anemometer Probe [43] .........................................45

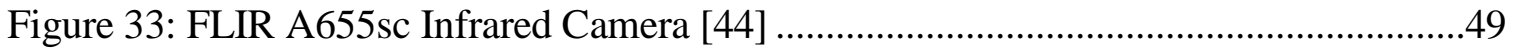

Figure 34: Camera Mounted during Transient Tests .........................................................51

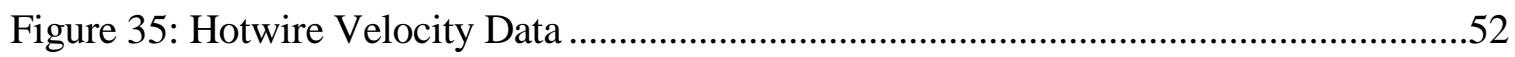

Figure 36: Velocity Power Spectral Density (PSD) from Hotwire Data .............................54

Figure 37: Autocorrelation Curve and Zoomed in Region of Interest (First Crossing)........55

Figure 38: Transient Convection Heat Transfer over a Semi-Infinite Solid Flat Plate.........57

Figure 39: Transient Film Cooling over a Semi-Infinite Solid Flat Plate ...........................59

Figure 40: FLIR IR Image with Thermocouple Locations ..............................................61

Figure 41: Film Cooling Effectiveness for Baseline Cases at Varying Blowing Ratio and Constant Turbulence Intensity.

Figure 42: Film Cooling Effectiveness for Baseline Cases at Constant Blowing Ratio and Varying Turbulence Intensity..... .68

Figure 43: Span-averaged Film Cooling Effectiveness for Current and Past Baseline

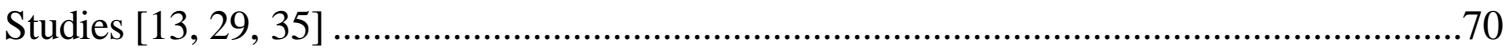

Figure 44: Span-averaged Film Cooling Effectiveness for Current Baseline Study ............71

Figure 45: Centerline Film Cooling Effectiveness of Baseline Case of Current and Past

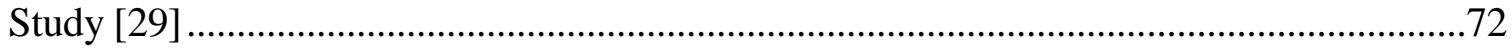

Figure 46: Centerline Film Cooling Effectiveness for Current Baseline Study...................72

Figure 47: Dimensionless Heat Transfer Coefficient of Baseline Case of Current and Past

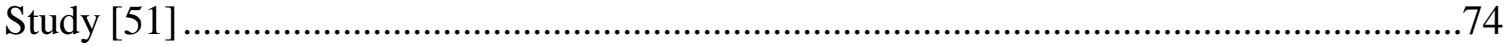

Figure 48: Three View Drawing of Anti-Vortex Hole Geometry ......................................75

Figure 49: Film Cooling Effectiveness Contour Plots of Varying Blowing Ratios at a

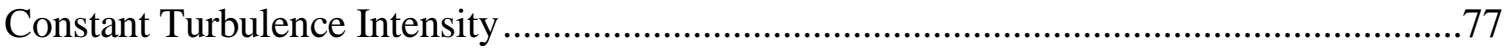

Figure 50: Film Cooling Effectiveness Contour Plots of Varying Turbulence Intensity with

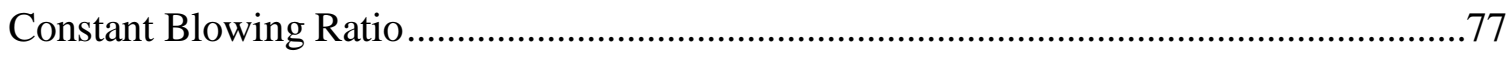

Figure 51: Span-averaged Film Cooling Effectiveness at Varying Blowing Ratio..............80

Figure 52: Span-averaged Film Cooling Effectiveness at Varying Turbulence Intensity ....81 
Figure 53: Centerline Film Cooling Effectiveness for Varying Blowing Ratio with Constant

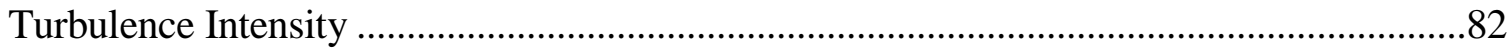

Figure 54: Centerline Film Cooling Effectiveness at Varying Turbulence Intensity ...........83

Figure 55: Span-Averaged Film Cooling Effectiveness of Anti-Vortex Hole [13].............86

Figure 56: Span-averaged Film Cooling Effectiveness of Current Study and Previous

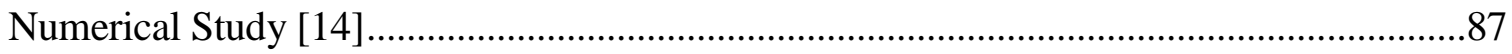

Figure 57: Span-averaged Dimensionless Heat Transfer Coefficient for Varying Turbulence Intensity .89

Figure 58: Span-averaged Dimensionless Heat Transfer Coefficient at Varying Blowing

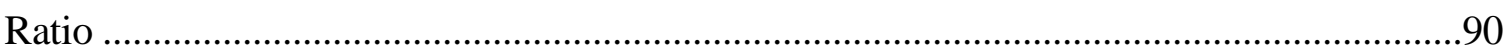

Figure 59: Span-Averaged NHFR at Varying Blowing Ratio and Constant Turbulence ....92 Figure 60: Span-averaged NHFR for Varying Turbulence Intensity .93

Figure 61: Centerline Dimensionless Heat Transfer Coefficient at Varying Blowing Ratio .94

Figure 62: Centerline Dimensionless Heat Transfer Coefficient for Varying Turbulence Intensity .95 


\section{NOMENCLATURE}
A
turbulence length scale constant
b
King's Law calibration constant, turbulence bar width
B
Meriam LFE constant
BR
blowing ratio or mass flux ratio $(\rho \mathrm{V})_{\mathrm{c}} /(\rho \mathrm{V})_{\infty}$
c
turbulence intensity constant
$\mathrm{c}_{\mathrm{p}}$
specific heat at constant pressure
C
Meriam LFE constant
D
main film cooling hole diameter
DR
density ratio $\rho_{\mathrm{c}} / \rho_{\infty}$
F
force
$\mathrm{H}$
heat transfer coefficient
$\mathrm{h}_{\mathrm{f}}$
film cooling heat transfer coefficient
K
thermal conductivity
$\mathrm{L}$
coolant hole length
M
mass flow rate
$\mathrm{N}$
number of frames
$\mathrm{Nu}$
Nusselt number
$\mathrm{P}$
pressure
Pr
Prandtl number
$\mathrm{q}_{\infty}$
dynamic pressure
$\mathrm{R}_{11}$
autocorrelation of the fluctuating velocity component
$\operatorname{Re}$
Reynolds number 


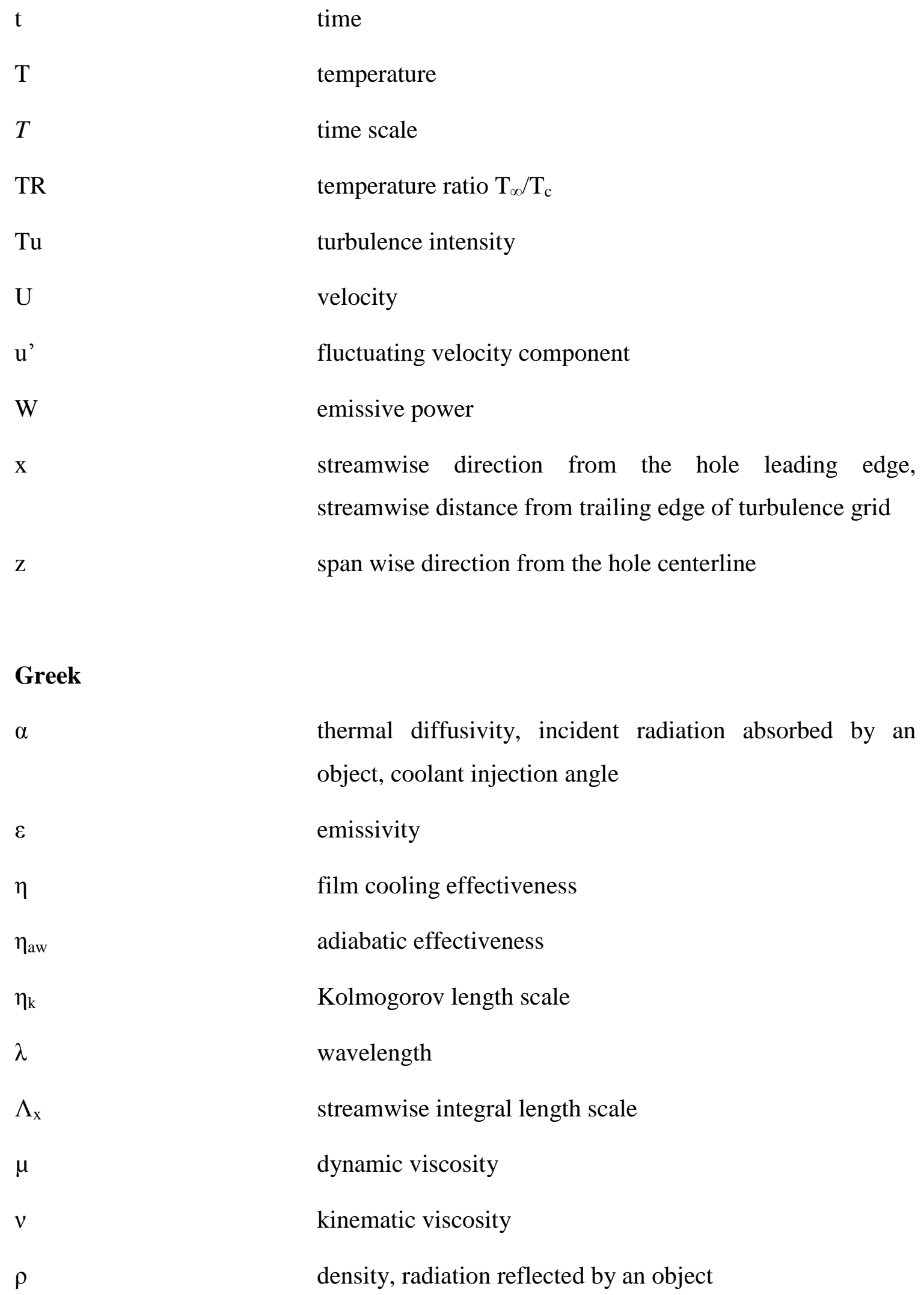

\section{Greek}

$\alpha$

$\varepsilon$

$\eta$

$\eta_{\text {aw }}$

$\eta_{\mathrm{k}}$

$\lambda$

$\Lambda_{\mathrm{x}}$

$\mu$

$v$

$\rho$

thermal diffusivity, incident radiation absorbed by an object, coolant injection angle

emissivity

film cooling effectiveness

adiabatic effectiveness

Kolmogorov length scale

wavelength

streamwise integral length scale

dynamic viscosity

kinematic viscosity

density, radiation reflected by an object 


\section{Subscripts}

aw

C

i

$\infty$

f

RMS

std

W

X

\section{Superscripts}

M

$\mathrm{N}$

\section{Abbreviations}

AVH

CAD

CFD

CRV

DAQ

IR radiation transmitted into an object, lag time

shear stress at the wall

adiabatic wall conditions

coolant conditions

coordinate direction (parallel with $\mathrm{x}$ ), pixel

freestream inlet conditions

film

root mean square

standard conditions

wall condition

streamwise distance turbulence length scale exponent

turbulence intensity decay exponent, King's Law exponent

anti-vortex hole

computer-aided design

computational fluid dynamics

counter rotating vortex

data acquisition

infrared 


\begin{tabular}{|c|c|}
\hline LDV & laser doppler velocimetry \\
\hline LED & light emitting diode \\
\hline LFE & laminar flow element \\
\hline LWIR & long wave infrared \\
\hline MWIR & mid wave infrared \\
\hline NHFR & net heat flux reduction \\
\hline NIR & near infrared \\
\hline PIV & particle image velocimetry \\
\hline PSD & power spectral density \\
\hline PSP & pressure sensitive paint \\
\hline RANS & Reynolds averaged Navier-Stokes \\
\hline $\mathrm{RBE}$ & radial blade exhauster \\
\hline RMS & root mean square \\
\hline SWIR & short wave infrared \\
\hline TLC & thermochromatic liquid crystals \\
\hline TSP & temperature sensitive paint \\
\hline VLWIR & very long wave infrared \\
\hline WVU & West Virginia University \\
\hline
\end{tabular}




\section{CHAPTER 1: INTRODUCTION}

\subsection{BACKGROUND ON GAS TURBINE ENGINES}

\subsubsection{EARLY HISTORY OF GAS TURBINE ENGINES}

Although the idea of the gas turbine engine dates back to approximately $130 \mathrm{BC}$, the true advent of the gas turbine is credited to John Barber who outlined the functionality of a gas turbine in his patent in 1791. This patent highlighted the key components necessary for a gas turbine engine, specifically the compressor, combustor and turbine sections $[1,2]$. Restricted by the low efficiency technology of the era, a working model of his patent was never realized.

Approximately 80 years later a German scientist, Dr. Franz Stolze, made significant strides from Barber's patent by way of his "fire turbine" shown in Figure 1. This machine was noteworthy because it was the first to have both compressor and turbine mounted on the same shaft. Ultimately, Stolze was limited by the engine efficiency, specifically the turbine inlet temperature, which is a key factor in governing engine efficiency, and will be elaborated upon later in this section.

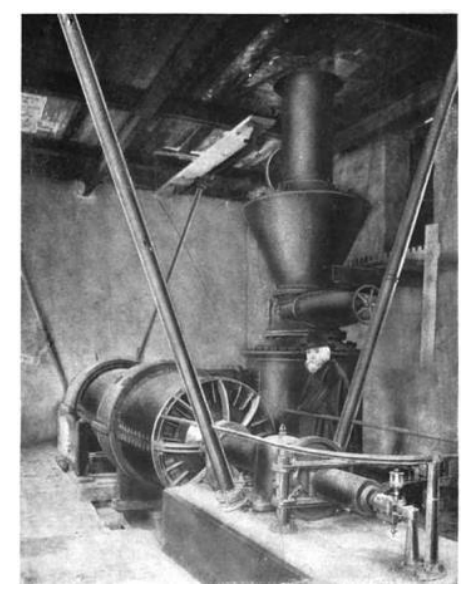

Figure 1: Dr. Franz Stolze's Fire Turbine [3] 
It wasn't until the 1930's that gas turbine engines really became a recognized and attainable technology. A British Royal Air Force cadet by the name of Frank Whittle was determined to design an engine that could attain higher efficiency than that of an aircraft propelled by an internal combustion engine driving a propeller. Whittle successfully tested his turbojet engine design in the late 1930's and in 1941 his engine was used to power the Gloster Meteor [4]. Simultaneously in Germany, Hans von Ohain, whom was unaware of Whittle's work, also saw a need for more efficient aircraft engines. His motivation was to have an engine that would have a higher power to weight ratio. His turbojet design was successfully test flown in a Heinkel HE 178 aircraft in 1939. Frank Whittle and Hans von Ohain actually did not meet until 1978.

Adolf Meyer of Brown Boveri in 1939 presented a technical paper on the history, progression, and possible future of gas turbine engines which coincided with the debut of the first land based turbine for power generation. This turbine was installed in Neuchatel, Switzerland and was operated until 2002 and was then moved to Birr, Switzerland where it remains on display [5].

\subsubsection{THE GAS TURBINE CYCLE}

Gas turbine engines are commonly used in aircraft and marine propulsion due to their high power to weight ratios, and have even seen implementation in locomotive and automotive sectors although are far less practical in the latter applications. Power generation turbines are also a practical use due to their high power density.

At their core, gas turbine engines are designed on the thermodynamic process known as the Brayton cycle, as can be seen in Figure 2 and in Figure 3a. This process describes the 
conditions through an ideal gas turbine engine cycle. The open Brayton cycle is comprised of four internally reversible processes which are as follows:

1) Isentropic compression via compressor

2) Constant-pressure heat addition via combustor

3) Isentropic expansion via turbine

4) Constant-pressure heat rejection
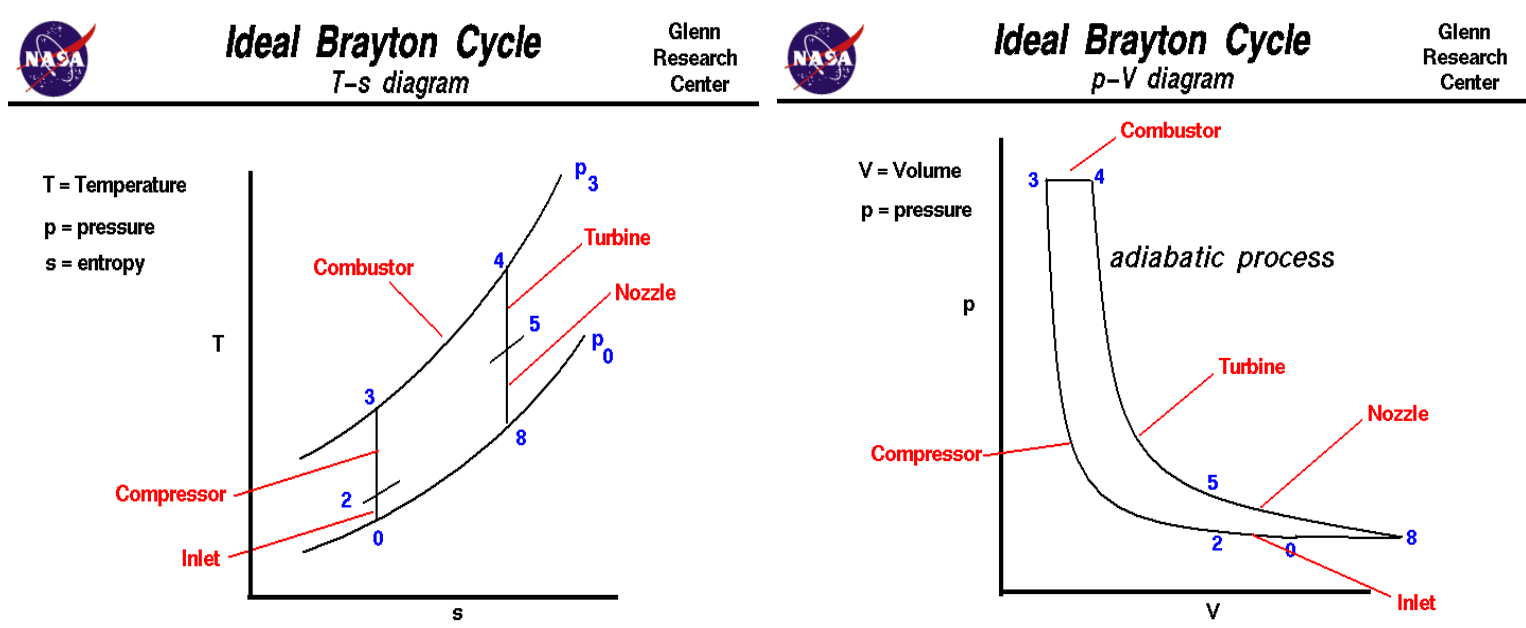

Figure 2: T-s and p-V Diagrams of Ideal Brayton Cycle [6]

In step one of this open cycle the working fluid (air) is drawn into the compressor and undergoes compression. Step two involves adding additional energy to the system through heat addition in the combustor by adding fuel to the system and then igniting it. If the combustor were not in the system the turbine could, at most, drive only the compressor resulting in no net work out of the system. The final step is expansion through the turbine which supplies energy to run the compressor and provides thrust in propulsion applications or provides energy to a steam power section in combined cycle power applications. However, in reality, irreversibilities such as friction losses, shaft losses, and a combination of heat and pressure losses exist in the Brayton cycle due to more work having to be done 
by the compressor and less work output being done by the turbine. Additionally, a pressure drop occurs during the heat addition process through the combustor.
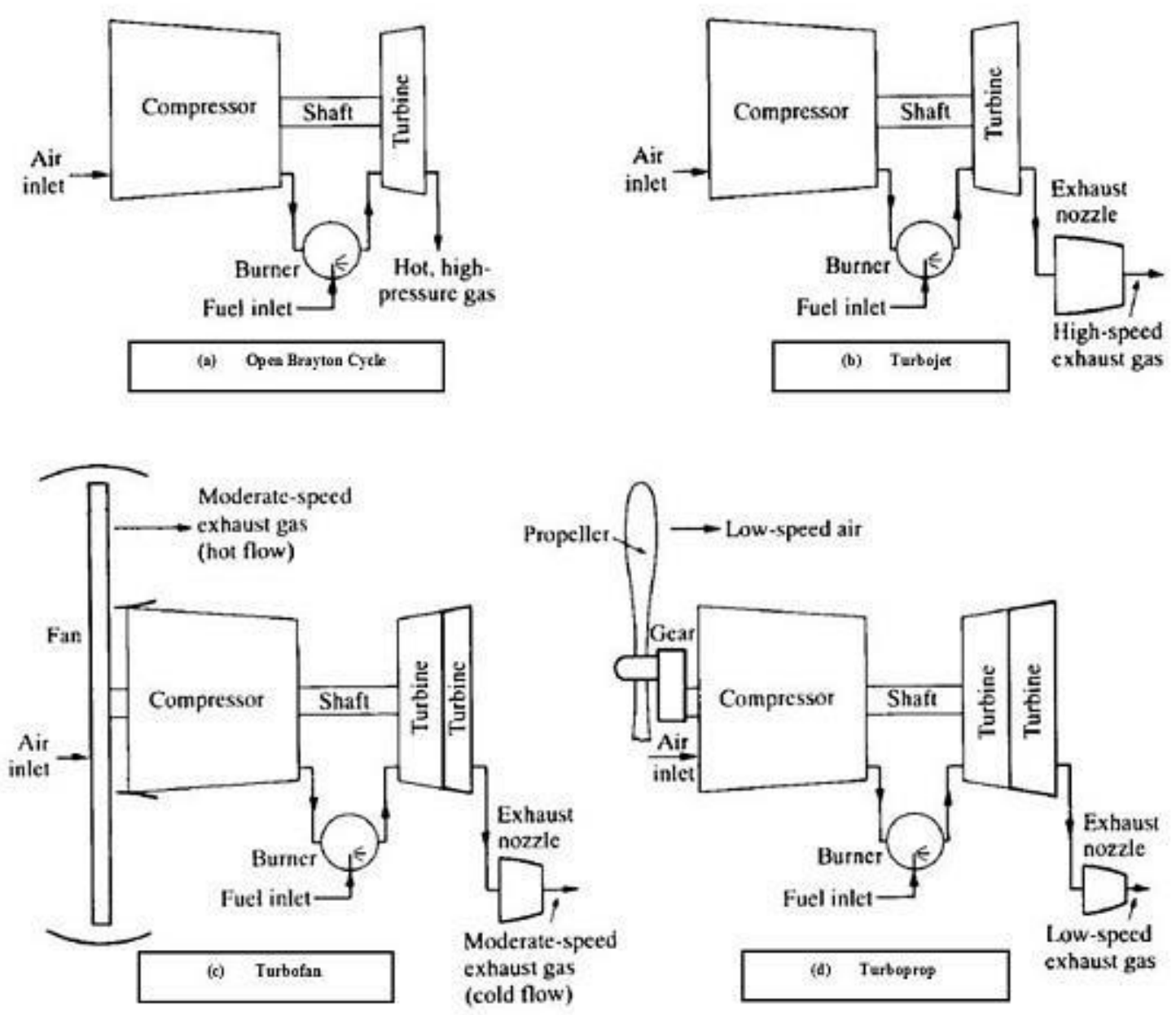

Figure 3: Schematics of Propulsion Systems [4]

There are three different types of aircraft engines based on the Brayton cycle. These are the turbojet, turboprop, and the turbofan respectively, as illustrated in Figure 3b-d and Figure 4 respectively. The turbojet engine was the first type of engine developed, where the thrust is solely produced from the exhaust gases being ducted and expanded through a nozzle creating a high velocity jet. This engine is ideal for high Mach number applications, and, with the addition of an afterburner, is used in supersonic flight. The turboprop engine 
is used in low Mach number applications due to the high propulsive efficiency that is achieved by the propeller. The turbofan engine may be thought of as a hybrid between a turbojet and turboprop engine. Thrust is produced through the core, which is a turbojet, and through a bypass to increase the mass flow rate of air at a lower velocity than through the core. This increases the propulsive efficiency of the engine and reduces engine noise and is widely used in commercial jet transport aircraft applications for these reasons.

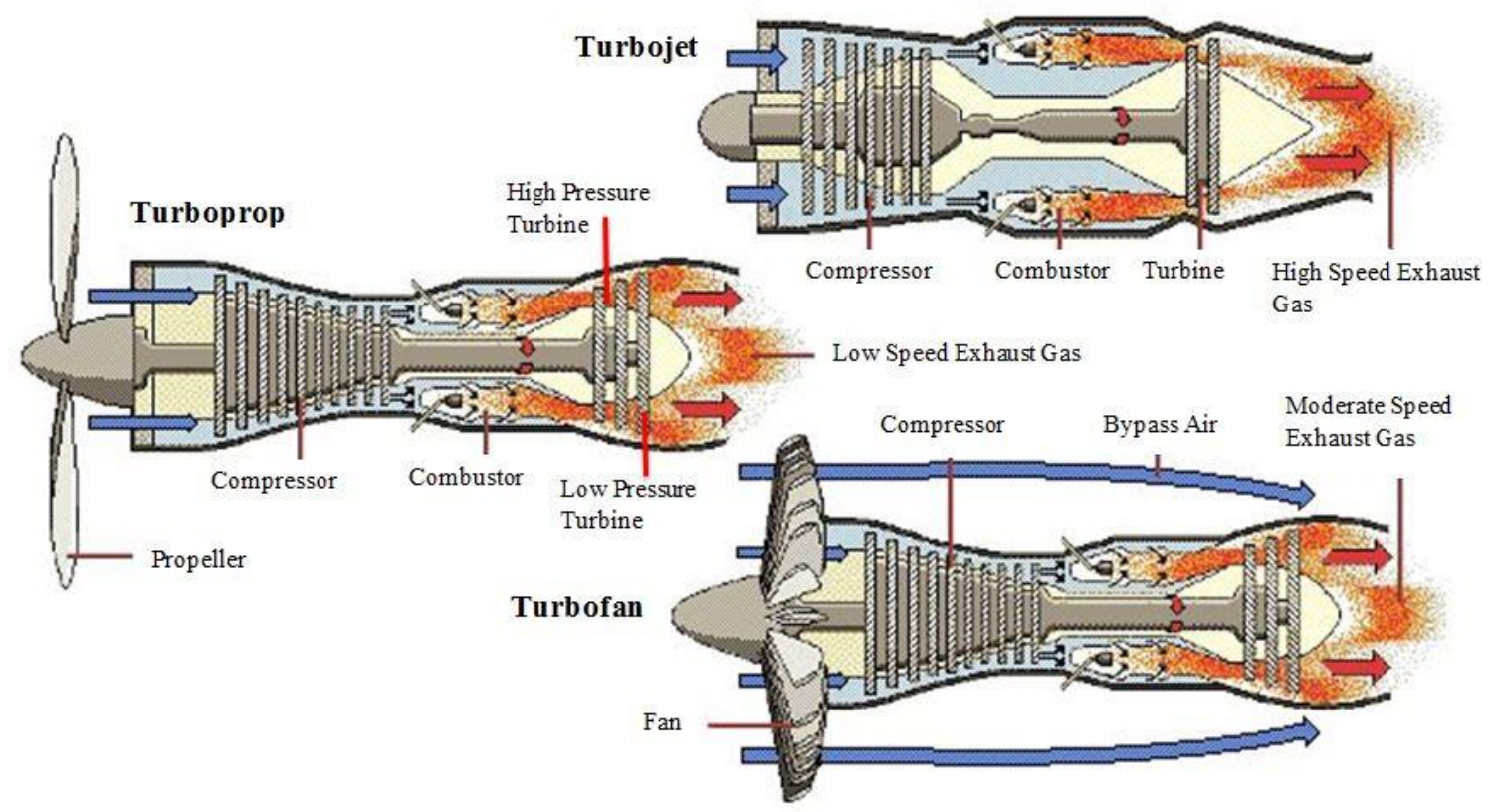

Figure 4: Diagram of Propulsion Systems [7] 


\subsection{HEAT TRANSFER AND COOLING IN GAS TURBINE ENGINES}

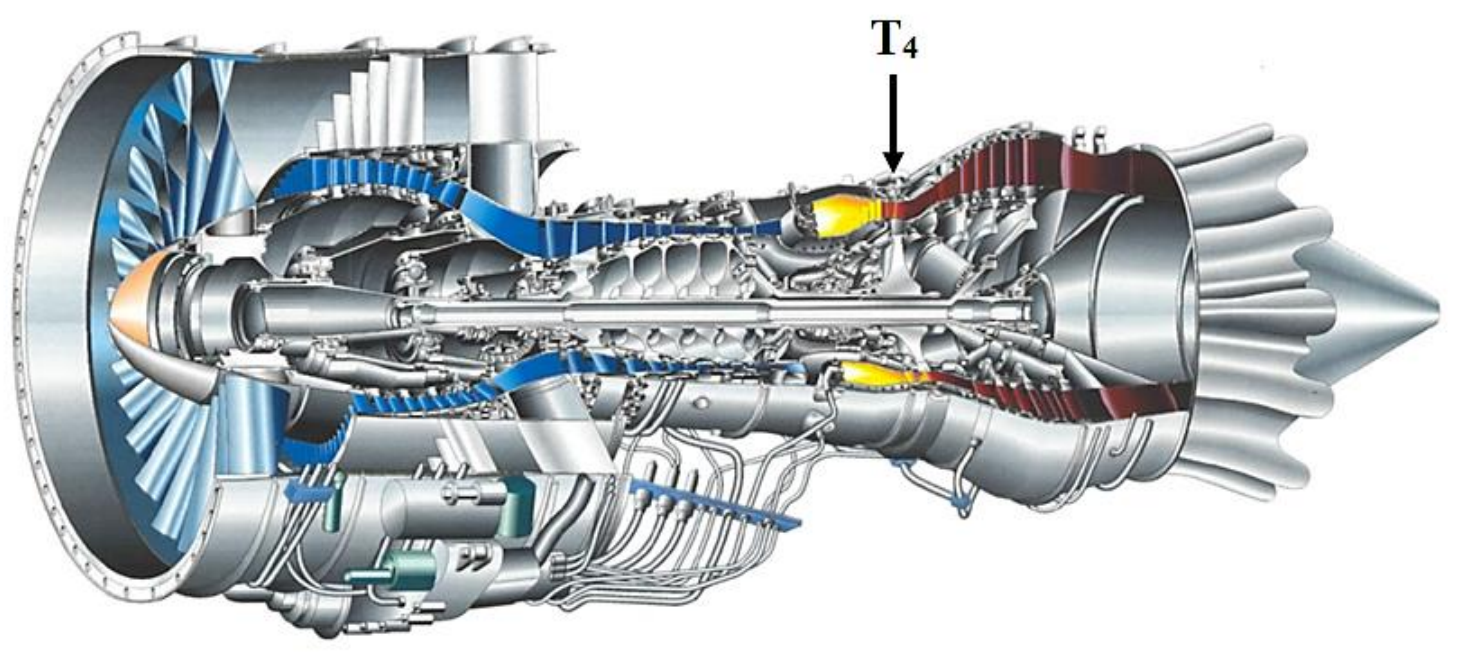

Figure 5: Turbofan Engine Showing Temperature Gradient of Air through Core [8]

Two primary ways to increase the efficiency of a gas turbine engine are to increase the compressor pressure ratio and to raise the combustor exit temperature, which is also referred to as the turbine inlet temperature and is commonly referred to as " $\mathrm{T}_{4}$ "; see Figure 2. Figure 5 displays the air path through the core of a turbofan engine where the cooler air is shown in a blue hue, and the hotter air beginning at " $\mathrm{T}_{4}$ " is shown in a red hue. The region where the red hue begins is the entrance of the turbine and is the region of interest for the subsequent cooling methods.

Although the concept of simply raising the turbine entrance temperature on the surface seems basic and straightforward, many problems arise from these higher temperatures. The melting point of the materials in the combustor and turbine sections are often exceeded by the freestream temperatures $(\approx 2000 \mathrm{~K})$ and the components can and will fail due to thermal creep, oxidation, hot corrosion, and thermal-mechanical fatigue if not adequately cooled. As a result, relatively cooler air from the compressor section upstream $(\approx 800 \mathrm{~K})$ must be extracted and used to cool the components in the combustor and turbine sections. The 
extraction of the air from the compressor section results in a lower overall engine efficiency, but the efficiency gained from the turbine section outweighs this loss if the cooling air is used effectively. The cooling of the components occurs both internally through cooling passages and externally through what is known as film cooling. Figure 6 displays multiple methods which have been implemented for turbine airfoil cooling.
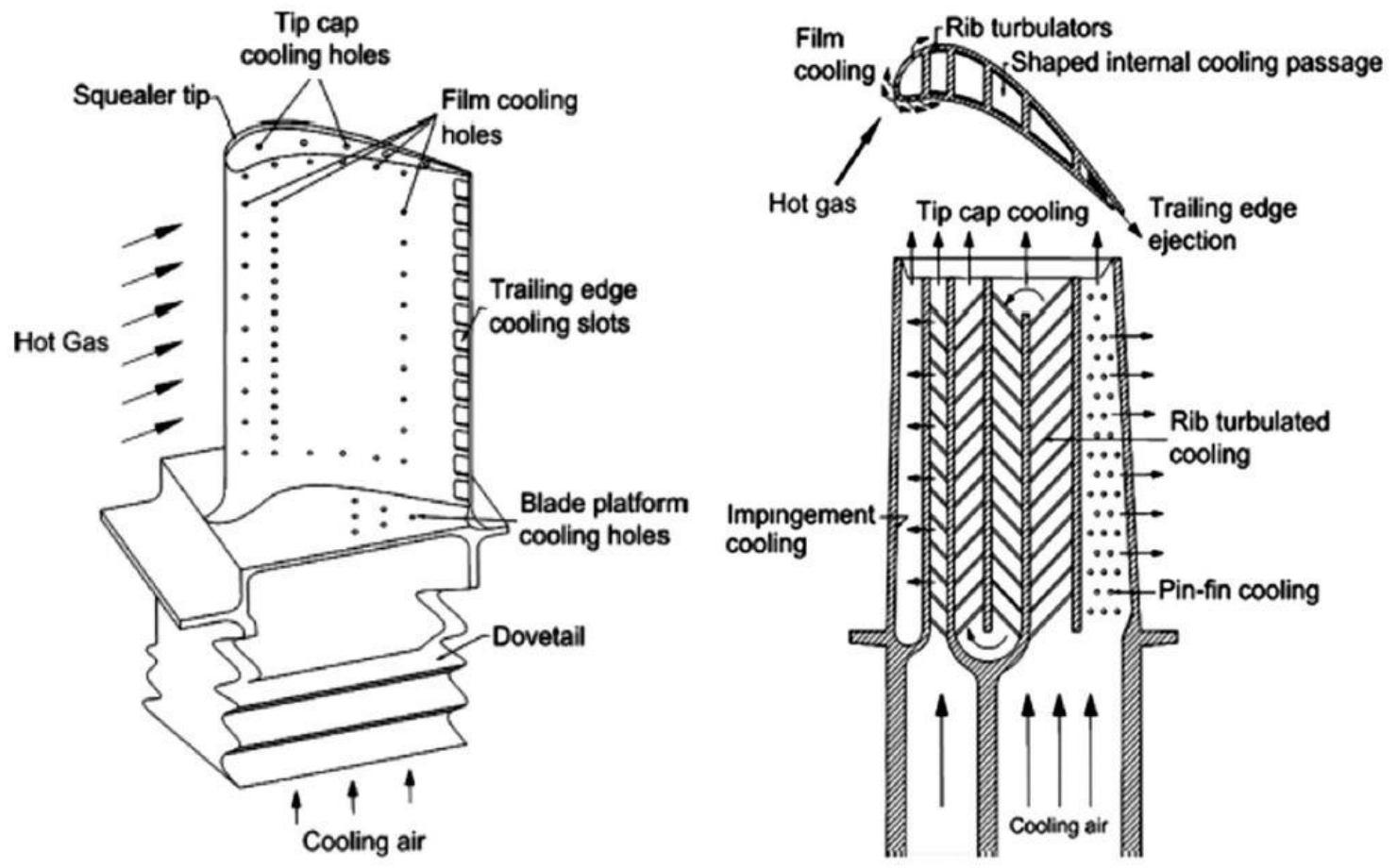

Figure 6: Gas Turbine Cooling Techniques [9]

\subsubsection{INTERNAL COOLING}

As discussed above, one method for cooling turbine airfoils is internal cooling. The coolant air in this application is introduced at the root of the airfoil and is routed through internal passages to cool the internal surfaces. The primary goal for internal cooling is to achieve a higher heat transfer coefficient by inducing turbulence to better draw heat from the internal passages through convection heat transfer. There are many different types of internal cooling, but only a few will be discussed here. 
Impingement cooling is used primarily at the leading edge of the turbine airfoil where the thermal loading is greatest. The internal cooling air functions by flowing perpendicularly and impinging on the internal passage that it is cooling, differentiating it from the following methods that use coolant flowing parallel or nearly parallel to the surfaces to be cooled. This method has the highest local heat transfer coefficient, but is limited spatially by the jet diameter.

The foremost internal cooling method is that of multi-pass serpentine passages routed through the majority of the airfoil. As previously stated, the coolant air enters through the root of the airfoil then passes through multiple turns until it is ejected as film coolant. These passages have enhancements in cooling with the addition of ribbed turbulators. These ribs are simply small walls that protrude into the coolant passage and provide enhancement of turbulence in the passage resulting in the promotion of convective heat transfer.

Dimple cooling is another option to use in internal passages that has been gaining momentum in recent years. Dimple cooling offers an alternative to pin-fin cooling by decreasing the pressure loss penalty in the inner channels and has a moderate heat transfer enhancement. As the flow passes over the dimples it detaches from the surface and reattaches with vortices enhancing the mixing and therefore, the heat transfer coefficient.

Pin-fin cooling is used within the trailing edge of the turbine airfoil to create turbulence to enhance the cooling. As the flow passes over the pins the flow separates and wakes are shed behind the pins. Alternating the spacing and staggering the pattern of the pins can enhance or diminish the performance of the design. The pins further serve as a conductive pathway to conduct heat away from the external surfaces. 


\subsubsection{EXTERNAL AND FILM COOLING}

As opposed to internal cooling, in external cooling, higher turbulence can be detrimental to the cooling of the components by inducing a higher heat load upon the surface. Film cooling is the process by which coolant air is ejected at discrete locations on the airfoil, creating a thin film layer of cooler air along the surface, insulating it from the hot freestream air. This method, when used at the leading edge of the airfoil, is referred to as showerhead cooling.

Film cooling protects the region directly downstream of the coolant holes and is used at the tip of the airfoil, at the platform/root of the airfoil, and through trailing edge slots. In efforts to optimize film cooling many different cooling hole geometries [11-13, 22, 25, 28$30,32,35-37]$ as well as other varieties of ideas have been investigated to maximize effectiveness or reduce the amount of coolant necessary. Thermal barrier coatings (TBC) have also been implemented in gas turbine engines to provide a coating over the airfoil. These materials can withstand a higher thermal loading and have been a large topic of interest in industry. TBCs are very low thermal conductive materials and are typically comprised of a metallic and a ceramic layer although alternatives of this exist.

Film cooling effectiveness $(\eta)$ is the non-dimensional parameter that represents how effective the coolant is at thermally protecting the surface from the freestream flow in theory. This value can fluctuate from zero (0) where the surface is equal in temperature to the freestream and one (1) where the surface is equal in temperature to the coolant.

\subsection{MEASURING TECHNIQUES OF FILM COOLING}

There are currently five different methods to measure film cooling effectiveness. One measurement technique is used to measure surface partial pressure and is known as 
pressure sensitive paint (PSP). The remaining four methods measure surface temperatures: thermocouples (point measurements), temperature sensitive paint (TSP), thermochromatic liquid crystals (TLC), and infrared (IR) cameras. All of these methods except thermocouples are aimed at measuring the surface in as a high of a spatial resolution as possible. By using the surface temperatures in conjunction with the freestream and coolant temperatures, eta can be determined.

\subsubsection{THERMOCOUPLES}

Thermocouples were the first temperature measuring device used in film cooling experimentation. Thermocouples are mounted flush with the surface to measure the temperature at a specific location and the wire leads are run through the test surface. Thermocouples are comprised of two different metals that are joined at a point and operate from the phenomenon known as the Seebeck Effect. A major drawback of thermocouples is that they can only offer measurements for discrete locations. Unless one was to create an entire surface out of thermocouples, which is highly impractical, then one is unable to completely understand the temperature distribution of the surface. Furthermore, thermocouples are an intrusive method and may disturb the flow passing over the surface.

\subsubsection{THERMOCHROMATIC LIQUID CRYSTALS (TLC)}

Thermochromatic liquid crystals are materials that change their color in the visible band of the electromagnetic spectrum (Figure 7) based on the amount of temperature change that the material undergoes and specifically translates to the film cooling effectiveness. A temperature change at each point on the surface results in a specific produced hue, saturation, and intensity. The surface must be illuminated by a white light to ensure proper functionality, and the hue produced from the color change is the driving 
factor in determining the temperature. TLC offers numerous different options depending on the desired application.

TLC also offers multiple temperature bandwidth options depending on the amount of temperature change the surface will undergo throughout the experiment. The most sensitive options offered are approximately $0.1^{\circ} \mathrm{C}$ and the larger bandwidths are around $30^{\circ} \mathrm{C}$ or more. TLC allows for continuous temperature change of the surface (up to the limits of the TLC material), so it is ideal for transient and steady state film cooling measurements. This application also offers a nonintrusive option which provides better accuracy than thermocouples and gives the temperature distribution of the entire surface. The process is also repeatable with no need to reapply the TLC between experimental trials.

The TLC method, although much more versatile than thermocouple measurement, is not without its own shortcomings. The surface must be cleaned and then a thin base layer coat of black paint must be applied before application of the TLC. The number of TLC layers applied as well as light or camera angles can result in different measurements, so if any of these variables change, a recalibration is required. In addition, if the TLCs experience temperatures in excess of their given bandwidth recalibration will be required. 


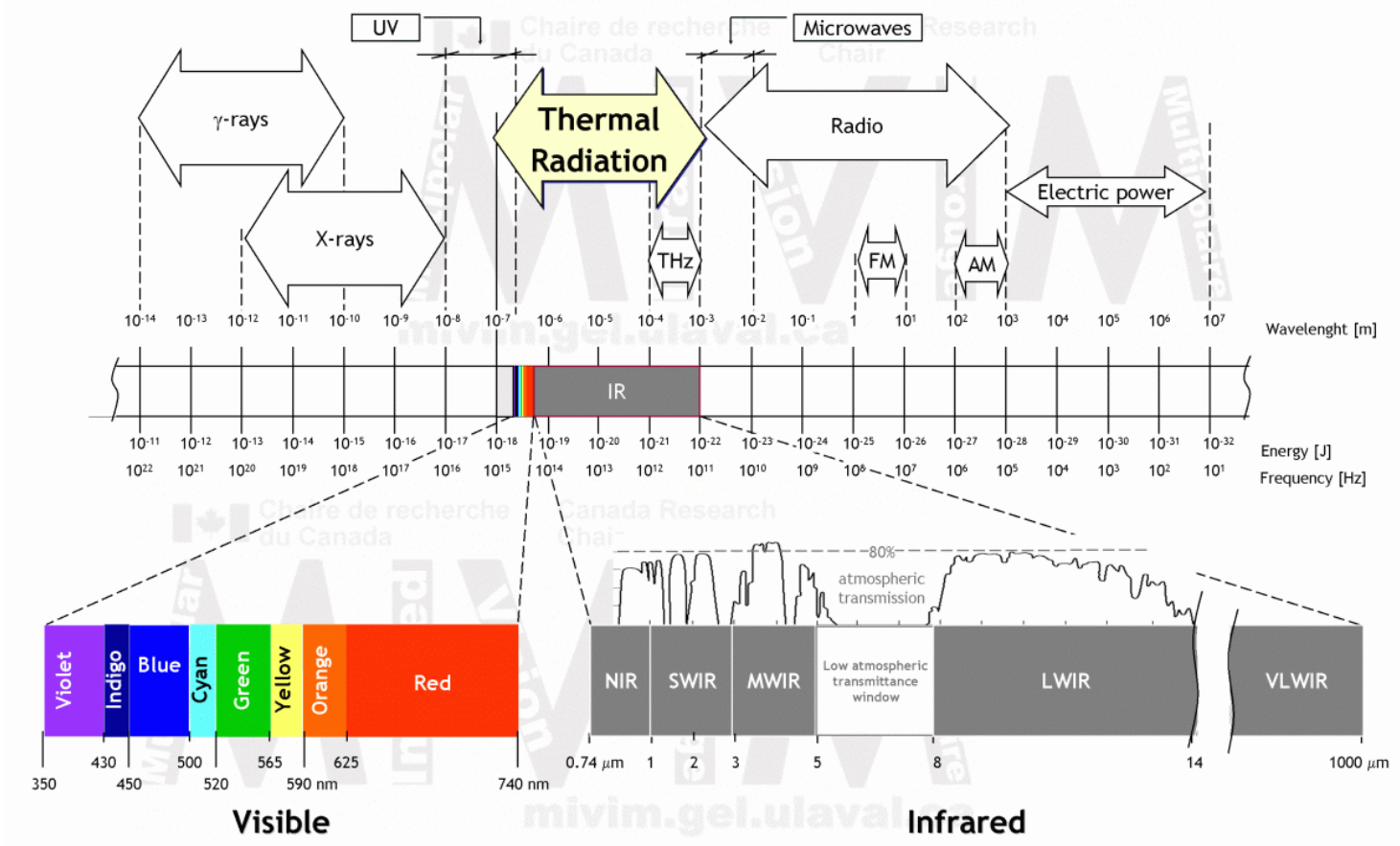

Figure 7: Electromagnetic Spectrum with Emphasized Visible and Infrared Bands [10]

\subsubsection{TEMPERATURE SENSITIVE PAINT (TSP)}

Temperature sensitive paint is another nonintrusive option for film cooling measurements that can be used in transient and steady state applications. The paint is comprised of luminescent molecules which are contained in a binder functioning from the principals of thermal quenching. A light emitting diode (LED) light is required to excite the paint by having it absorb photons. When the paint heats up, photons are released and results in the paint having a lower, known intensity correlating to a surface temperature measurement. As with the TLC method, TSP falls in the visible band of the electromagnetic spectrum, but offers a larger bandwidth than TLCs and is often used for that reason. TSP only functions with an increasing temperature and is only serviceable for one experiment and must be reapplied for each new experiment. 


\subsubsection{PRESSURE SENSITIVE PAINT (PSP)}

Pressure sensitive paint is very similar to TSP in that luminescent molecules are contained in a binder, but for the case of PSP, it is an oxygen permeable binder. This mass transfer method operates on the process of oxygen quenching and has the added benefit of determining film cooling effectiveness without the effects of conduction. Again the paint must be excited with photons provided by a LED source and as the pressure on the surface increases photons are released resulting in lower intensities in the higher pressure locations. These intensities are used in conjunction with a calibration curve to measure the film cooling effectiveness. Using the measured local surface pressure, a local skin friction coefficient can be determined as well as a local flow velocity. When used with the Prandtl (Pr) and Stanton (St) numbers a heat transfer coefficient can be calculated. PSP must be reapplied after each experiment and requires the use of two gases with different densities. PSP is the preferred method if one only chooses to look at film cooling and not the heat transfer coefficient because no error due to conduction will occur.

\subsubsection{INFRARED (IR) THERMOGRAPHY}

Infrared thermography is the method used in the current study and consequently will be elaborated upon to a larger degree than the prior methods. IR thermography offers a nonintrusive option that requires little to no surface preparation. This method, like the TSP and TLC methods, gives the temperature distribution of the entire surface. IR cameras also offer a much larger temperature bandwidth with high accuracy and reliability if properly calibrated or if used in situ with thermocouples. IR cameras also offer the additional benefit from the other techniques by recording the initial temperature distribution prior to 
transience. The major drawback of the IR camera is that its cost is much more than all of the aforementioned options.

In contrast to TLC, PSP, and TSP, infrared cameras work in the thermal band of the electromagnetic spectrum rather than the visible band which ranges from approximately $305 \mathrm{~nm}$ to $740 \mathrm{~nm}$. The thermal band can be separated into five smaller approximate bands: near infrared (NIR) with a wavelength range of $0.7-1.0 \mu \mathrm{m}$, short wave infrared (SWIR) with a wavelength range of 1.0-3.0 $\mu \mathrm{m}$, mid wave infrared (MWIR) ranging from 3.0$5.0 \mu \mathrm{m}$, long wave infrared (LWIR) ranging from 7.0-14.0 $\mu \mathrm{m}$ and very long wave infrared (VLWIR) with a range from $14.0-1000 \mu \mathrm{m}$. Figure 7 displays the entirety of the electromagnetic spectrum with special emphasis on the visual and thermal bands.

\subsection{OVERVIEW OF CURRENT WORK}

\subsubsection{DESIGN, FABRICATION, AND BENCHMARKING OF NEW FACILITY}

To the author's knowledge, the present experimental study is the first of this type being conducted at West Virginia University (WVU). An experimental facility had to be designed and fabricated by the author to perform desired experimentation as covered in 1.5.2 and will be discussed in Chapter 2. There is a vast amount of open literature on flat plate film cooling for experimental values to compare in regards to straight hole or cylindrical geometries. This necessitated a straight hole geometry to be investigated to benchmark and ensure that the facility was functioning accurately.

\subsubsection{EVALUATION OF AN ANTI-VORTEX GEOMETRY}

The main objective of this research work was to experimentally investigate how various freestream turbulence levels influence the performance of an anti-vortex cooling hole 
$(\mathrm{AVH})$ geometry designed and evaluated by previous researchers Heidmann and Ekkad [11] and Heidmann [12], Dhungel et al. [13], and Hussein et al. [14]. Previous examination of this geometry occurred only at low freestream turbulence levels which does not accurately portray realistic engine conditions where freestream turbulence may be as high as $25-30 \%$ [15]. Previous research [11-13] demonstrates that at higher blowing ratios and low turbulence the AVH design can diminish or eliminate the vorticity induced by the traditional straight hole. The present experimental trials conducted were for four different blowing ratios: $0.5,1.0,1.5$, and 2.0 with three freestream turbulence levels of $1,7.5$, and 11.7\%. Each test was performed a total of four times with two similar experiments being conducted on the same day, and then twice again on a separate day under alternate atmospheric conditions to ensure reliability of the facility.

\subsection{LITERATURE REVIEW}

\subsubsection{TEMPERATURE EFFECTS ON COMPONENTS}

Section 1.2 outlined that the turbine inlet temperature was a key driving factor to increase the thermal efficiency, and thus, the overall efficiency of the engine. With the temperature of the turbine inlet reaching well above the melting point of the superalloys used in the turbine section, film cooling becomes crucial. The average increase in turbine inlet temperature over the last 20 years has been $8 \mathrm{~K}$ per year. The general rule used by engine designers is that a $10 \mathrm{~K}$ rise in temperature will reduce the life of the component by $50 \%$ [16]. Figure 8 displays the history of turbine inlet temperatures with the introduction of cooling methods dating back to 1950 . 


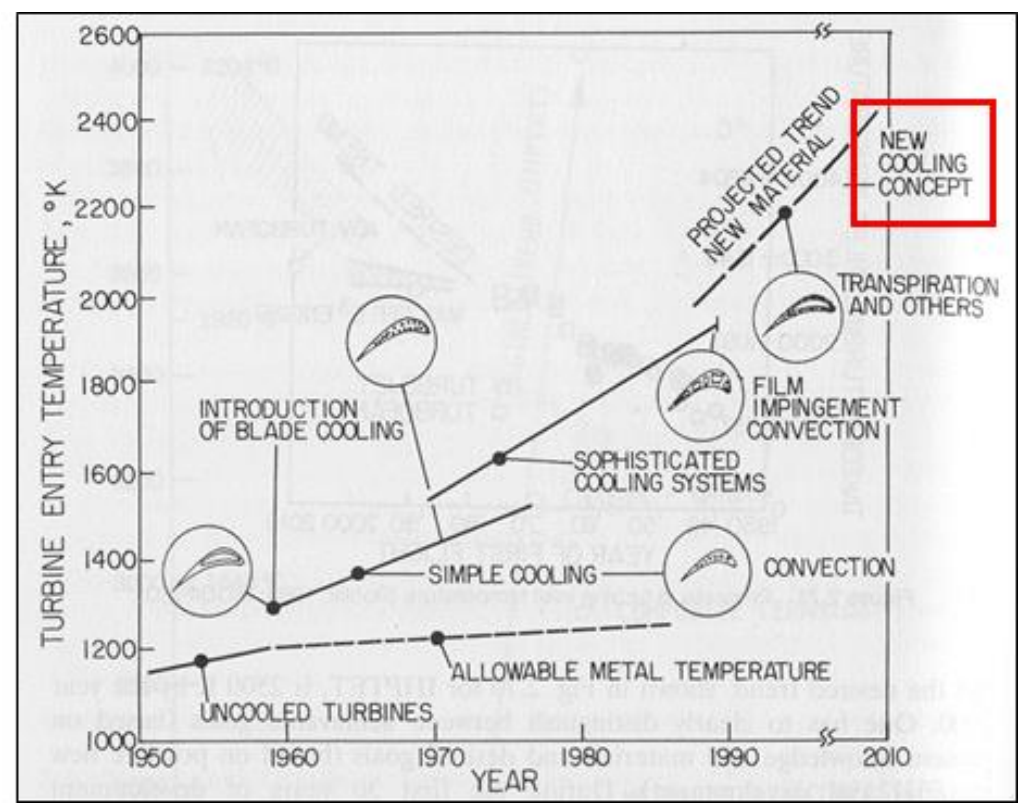

Figure 8: Rise in Turbine Inlet Temperature throughout History [17]

\subsubsection{FILM COOLING STUDIES}

While the pressure and suction surfaces of a gas turbine airfoil have a concave and convex curvature respectively, flat plate studies of film cooling effectiveness can be performed by non-dimensionally matching conditions and enlarging the area of interest many times. These studies can be applied accurately, with corrections, to valid engine conditions to predict film cooling effectiveness. It should be noted that many studies have been conducted using non-dimensionalized airfoils, but a majority of the research as it pertains to heat transfer and film cooling has been conducted using flat plates and as such will be the main focus of this section, as the current research was also conducted on flat plates.

Flat plate studies have investigated changing a variety of flow parameters such as, but not limited to: blowing ratio (BR), density ratio (DR), temperature ratio (TR), Reynolds number based on hole diameter, and freestream turbulence intensity (Tu). Additionally, cooling hole geometries and cooling hole parameters: hole pitch $(\mathrm{P})$ and hole length $(\mathrm{L})$ 
both non-dimensionalized by hole diameter (D) and coolant injection angle $(\alpha)$ and variations of exit geometry have been investigated to evaluate their effect on adiabatic cooling effectiveness $\left(\eta_{\mathrm{aw}}\right)$. Adiabatic film cooling effectiveness is defined as:

$$
\eta_{\mathrm{aw}}=\frac{\mathrm{T}_{\infty}-\mathrm{T}_{\mathrm{aw}}}{\mathrm{T}_{\infty}-\mathrm{T}_{\mathrm{c}}}
$$

Here $\left(T_{\infty}\right)$ is the freestream flow temperature, $\left(T_{c}\right)$ is the coolant flow temperature, $\left(T_{a w}\right)$ is the adiabatic wall temperature, and $\left(\eta_{\mathrm{aw}}\right)$ is the film cooling effectiveness for an adiabatic wall. Adiabatic film cooling measurements are used for low temperature testing (relative to engine conditions) and do not account for any heat loads due to radiation. Adiabatic wall temperature is the case of a perfectly insulated wall where no heat transfer is existent, and the only transfer of energy present is in the form of work.

\subsubsection{CONVENTIONAL STRAIGHT HOLE STUDIES}

Extensive research has been conducted on the conventional and simple straight hole geometry. Kadotani and Goldstein [18, 19] determined that the freestream turbulence intensity, blowing ratio, and the ratios of turbulence length scale to hole diameter and length scale to boundary layer thickness were of the greatest importance when studying the variation of film cooling effectiveness. Blowing ratio, density ratio, and Reynolds number based on main cooling hole diameter can be defined as follows:

$$
\begin{gathered}
\mathrm{BR}=\frac{\rho_{\mathrm{c}} \mathrm{U}_{\mathrm{c}}}{\rho_{\infty} \mathrm{U}_{\infty}} \\
\mathrm{DR}=\frac{\rho_{\mathrm{c}}}{\rho_{\infty}} \\
\operatorname{Re}_{\mathrm{D}}=\frac{\rho_{\infty} \mathrm{U}_{\infty} \mathrm{D}}{\mu_{\infty}}
\end{gathered}
$$


Here $\left(\rho_{c}\right)$ is the coolant density, $\left(\rho_{\infty}\right)$ is the freestream density, $\left(U_{c}\right)$ is the coolant velocity exiting the holes, $\left(\mathrm{U}_{\infty}\right)$ is the freestream velocity, (D) is the main cooling hole diameter, and $(\mu)$ is the freestream dynamic viscosity.

Han and Mehendale [20] found that the optimum blowing ratio for a straight hole geometry is between 0.5 and 0.7 for a single row of straight cooling holes. This range of blowing ratios is the approximate maximum ratio that can be achieved before jet lift off occurs and consequential detachment of the coolant jet from the airfoil surface. In real engine applications, the blowing ratios are at a minimum approximately 1.5 and are often well above this value. When jet lift off occurs at higher blowing ratios the coolant jet, near the exit of the hole, acts similarly to a solid cylinder in a cross flow as it enters the freestream and the hot freestream gases pass around the coolant jet. Haven et al. [21] described and illustrated in detail, that a phenomenon known as a counter-rotating vortex (CRV) pair forms as can be seen in Figure 9. When this occurs the downwash of the CRV draws the hot freestream flow and entrains it upon the surface. As the vortex continues through its progression the coolant is drawn away from the surface due to the upwash from the internal portion of the CRV pair, further reducing the effectiveness of the coolant jet.

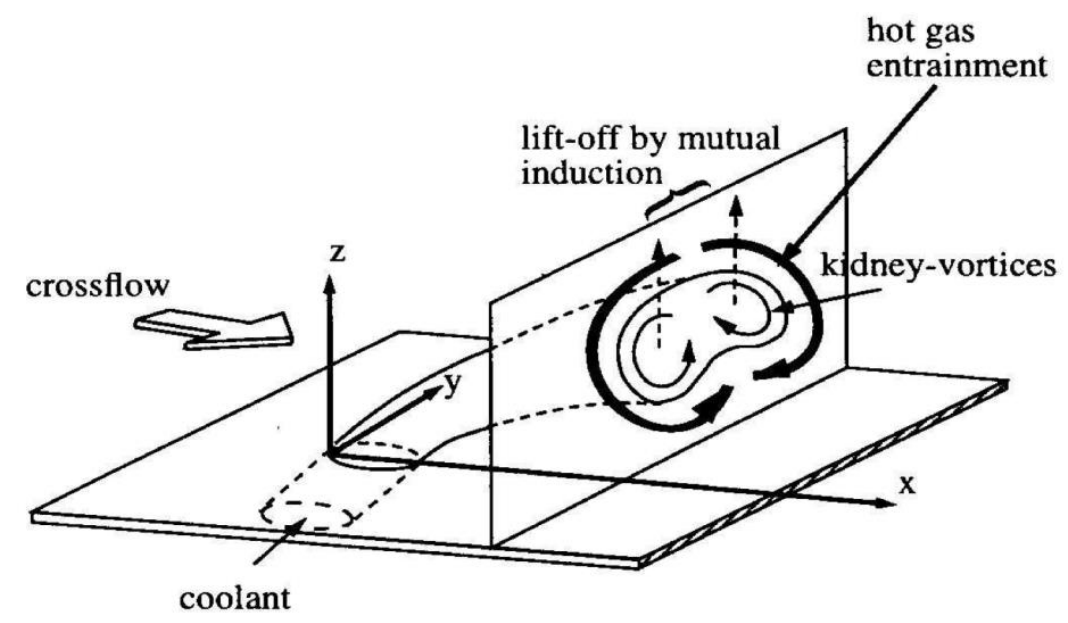

Figure 9: Counter-rotating Vortex Pair and Jet Lift off from Haven et al. [21] 
Kohli and Bogard [22] considered a large angle of inclination $\left(55^{\circ}\right)$ of a straight cooling hole geometry versus a $35^{\circ}$ inclination and found that both the centerline and spanaveraged effectiveness values were lower for the higher angles of injection at all blowing ratios for low turbulence cases.

Bons, et al. [23] studied the effects of freestream turbulence for a row of straight holes inclined at $35^{\circ}$ for multiple blowing ratios and found that centerline effectiveness is decreased with increasing turbulence, but midline effectiveness (between the holes) is increased with an increasing turbulence.

Lawson and Thole [24] studied surface deposition effects on a $30^{\circ}$ inclined straight hole geometry and prior to testing the deposition, investigated turbulence on a low blowing ratio case $(\mathrm{BR} \approx 0.49)$ for freestream turbulence intensities of $4.6 \%$ to compare to previous studies, and a $12.3 \%$ case as a benchmark for subsequent deposition testing. For the highest turbulence case the laterally averaged effectiveness was higher for non-dimensional streamwise location (x/D) up to an $x / D$ of 5 and was essentially the same as the $4.6 \%$ case for larger $\mathrm{x} / \mathrm{D}$. The centerline effectiveness for the $12.3 \%$ case up to an $\mathrm{x} / \mathrm{d}$ of 3 was higher than that of the $4.6 \%$ case, but was noticeably lower for larger x/D locations.

\subsubsection{SHAPED AND ADVANCED COOLING HOLE GEOMETRIES}

The introduction of advanced cooling hole concepts comes with the ambition to decrease the exit jet momentum of the coolant leaving the hole so that lift off will be decreased or diminished and to deplete or entirely mitigate the formation and existence of the CRV pair. Schmidt, et al. [25] investigated $60^{\circ}$ compound angle straight holes where the flow is injected at an angle to the freestream rather than parallel to it. They performed this for a density ratio of 1.6 that they achieved through cryogenically cooling the coolant flow. 
They found that at low blowing ratios the near hole region of the compound angle hole had a higher effectiveness, but overall had approximately the same effectiveness as the conventional straight hole. However for higher blowing ratios, they established that the compound hole was much more effective due to lateral spreading of the jet. Ekkad, et al. [26] studied the effect of density ratios of 0.98 and 1.46 on heat transfer coefficient for conventional straight, $45^{\circ}$, and $90^{\circ}$ compound angle holes at an elevated turbulence level of $\approx 8.5 \%$. They found in general that increasing the blowing ratio in turn increased the heat transfer coefficient. Of additional interest, they found that the heat transfer coefficient decreases with an increasing density ratio due to a decrease in jet lift off. It should be noted that the density ratio experienced in a gas turbine engine is often greater than 1.5 and is typically closer to 2.0 .

Bunker [27] presents a review of some of the shaped coolant hole geometries that had been studied up until 2005, and can be seen in Figure 10. Geometry A is a "classic" shaped hole that provides lateral expansion referred to as fan-shaped and provides expansion into the surface commonly referred to as laidback. Geometry B only has the fan-shaped expansion, geometry $\mathrm{C}$ only has laidback expansion, and geometry $\mathrm{D}$ expands from the root of the cooling hole both forward and rearwards equally and is referred to as conical. 


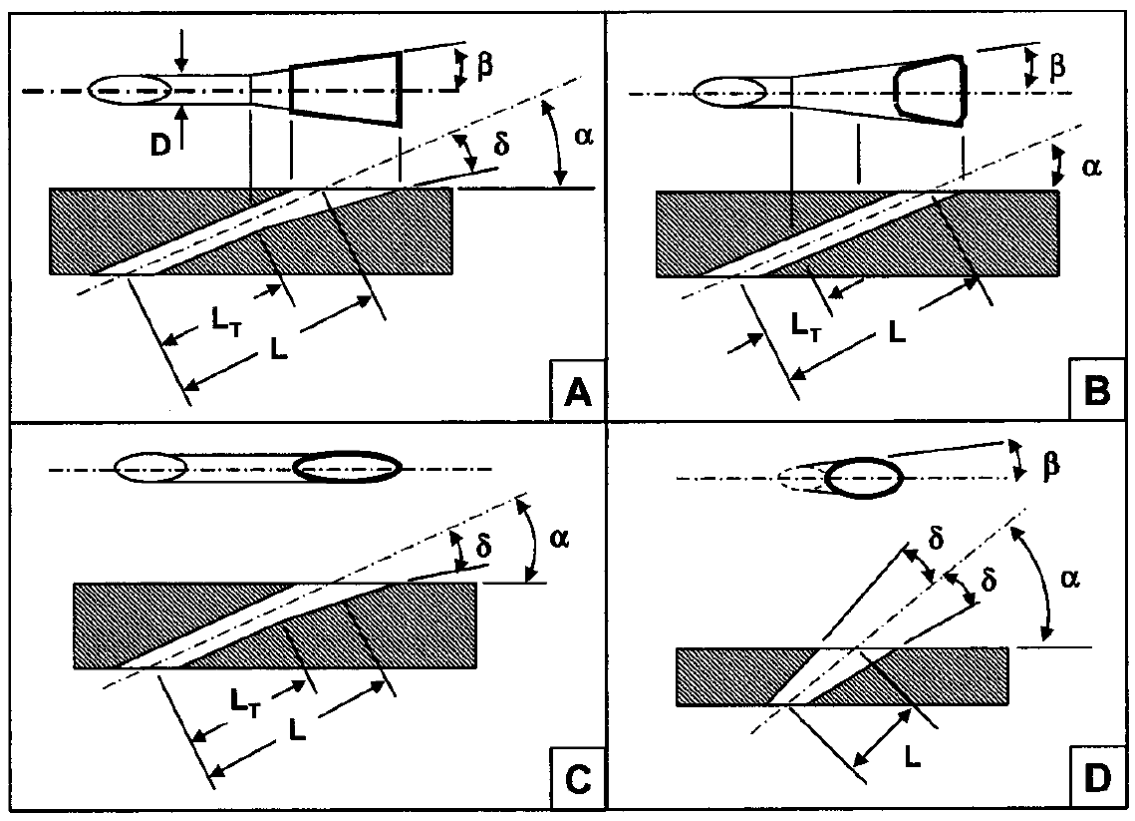

Figure 10: Four Types of Shaped Film Cooling Holes [27]

Gritsch, et al. [28] considered the effectiveness of fan-shaped and laidback fanshaped holes and how their performance compared to that of conventional straight holes for low turbulence levels $(\approx 1 \%)$. They found that both fan-shaped geometries showed significant improvement of thermal protection particularly at higher blowing ratios. The fan-shaped geometries allow the coolant air to be injected at a lower velocity than the straight hole due to the larger exit area and as a result have a better lateral spreading than the straight hole geometry. Wright, et al. [29] studied how varying the density ratio and turbulence intensity changed the film cooling effectiveness for cylindrical, fan-shaped, and fan-shaped laidback holes. They were able to ascertain that for straight cooling holes at lower blowing ratio a density ratio of 1.0 was superior to a ratio 1.4 , but at greater blowing ratios the higher density ratio performed better. The shaped holes however performed better at all blowing ratios for a density ratio of 1.0. In all cases an increase in freestream turbulence resulted in a decrease in film cooling effectiveness. 
Saumweber, et al. [30] investigated freestream turbulence effects on cylindrical, fanshaped, and laidback fan-shaped holes for freestream turbulence intensities of 3.6, 7.5, and $11 \%$. It was found that effectiveness for straight hole cases is reduced for low blowing ratio as the turbulence increases, but for higher blowing ratio the film cooling effectiveness is noted as having a slight improvement at higher turbulence intensities due to the mixing of the coolant. Contrary to the straight hole cases, the fan-shaped geometries experienced a loss of effectiveness at all blowing ratios as the turbulence intensity increased.

Takeishi, et al. [31] present a method for straight and laidback geometries that utilizes swirling coolant flow to combat the formation of the CRV pair. To induce the swirling of the coolant, two impingement jets are located at the entrance of the coolant hole with varying slant angles. For the straight hole configuration a higher film cooling effectiveness was realized, as jet lift off was reduced and the flow remained attached to the surface. Furthermore, the CRV was diminished almost entirely. For fan-shaped holes the coolant jet spread wider laterally than the non-swirling case, and a higher film cooling effectiveness was realized. Although the results of this method are certainly promising and should be investigated further, current technology and the far smaller scale that must be achieved in an actual engine condition prevent this from being a practical method.

Lu, et al. [32] investigated spacing straight holes farther apart and embedding the hole exits in a trench. They further investigated four different configurations of trenches against the conventional straight hole case for medium turbulence $(\mathrm{Tu} \approx 6 \%)$ as can be seen in Figure 11. The shaded regions resemble the part of the trench that had been replaced compared to the large rectangular trench in case 1 . They found that in all cases the trenched geometries performed better than the conventional case because the coolant spread laterally 
in the trench before flowing downstream. Cases 2 and 4 showed the most promising spanaveraged effectiveness. The proposed method of creating the trench would occur during the application of the TBC coating on the airfoil.

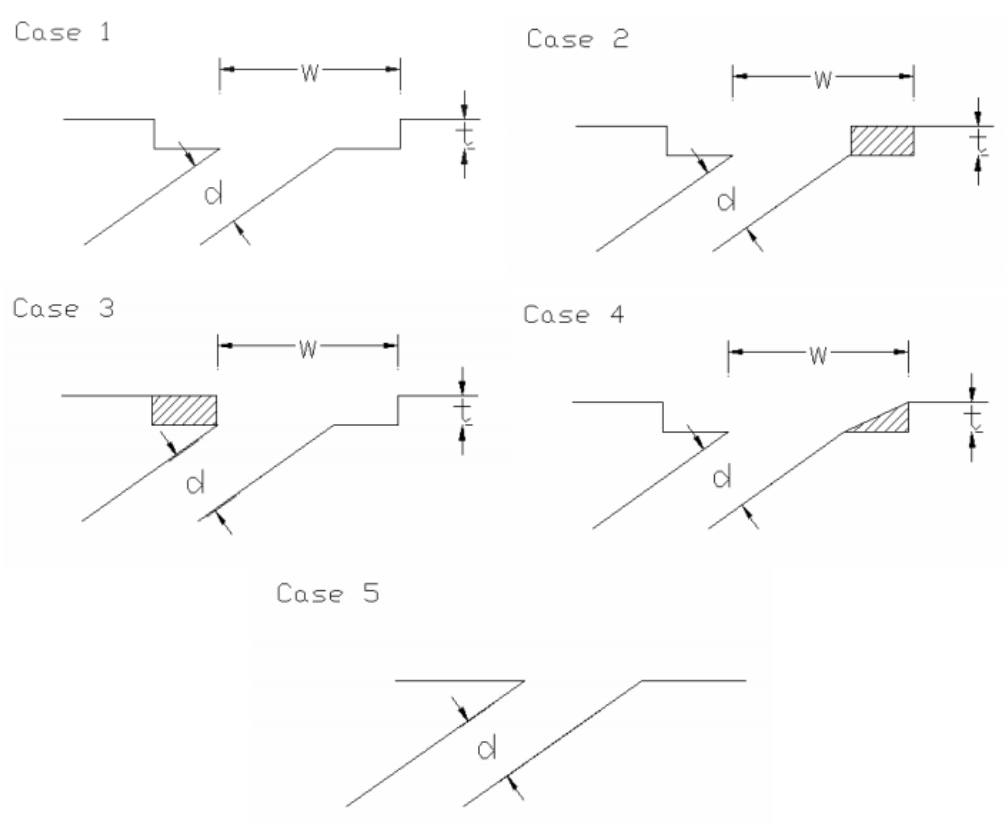

Figure 11: Four Trench Configurations with Conventional Straight Hole [32]

Barigozzi, et al. [33] investigated a possible application of an upstream ramp to see how it would improve adiabatic effectiveness. The two geometries that they selected to use were the cylindrical and fan-shaped with conical expanded exit. Their experiment had a range of blowing ratios from 0.3-1.0, with low turbulence intensity. It was established that at low blowing ratios the cylindrical hole had better lateral diffusion with the addition of the ramp. The fan-shaped hole with the addition of a ramp showed diminished effectiveness for all blowing ratios due to the ramp creating intense mixing. This method raises concern over the manufacturing cost, as well as the possibility of the ramp protruding into the freestream and failing from thermal loading. 
Ely and Jurban [34] proposed and numerically investigated a sister hole cooling concept and compared their results to cylindrical holes. The sister hole concept has two smaller holes along the main coolant hole that have their own separate hole entrances from the plenum which has the added benefit of being much more feasible to manufacture than other methods. The idea behind this design is that the CRV pairs resulting from the sister holes will weaken the CRV pair from the main hole by counteracting the downwash of the main hole with an upwash from the sister holes. The numerical investigation was performed on blowing ratios from $0.2-1.5$ with a freestream turbulence of $\approx 2 \%$. It was concluded that at all blowing ratios the centerline of the main cooling hole and spanaveraged effectiveness were improved over the cylindrical hole geometry.

\subsubsection{ANTI-VORTEX COOLING HOLE GEOMETRY}

Heidmann [11] developed a geometry known as the anti-vortex hole design which is a similar concept to the sister hole design where it utilizes secondary holes to counteract the main cooling hole CRV pair as can be seen in Figure 12. The overarching difference between the AVH concepts as opposed to the former is that the side cooling holes share the air from the main coolant hole by intersecting it. This is beneficial for two reasons: the secondary hole CRV pairs will counter the main cooling hole CRV pair resembling the sister hole design, but the coolant air will be diffused through the secondary holes, reducing jet lift off for the main cooling hole. Through optimization the AVH design is a strong candidate to entirely mitigate the CRV pair at the main hole. Numerical investigation of the $\mathrm{AVH}$ design predicted significant improvement over the cylindrical geometry. 


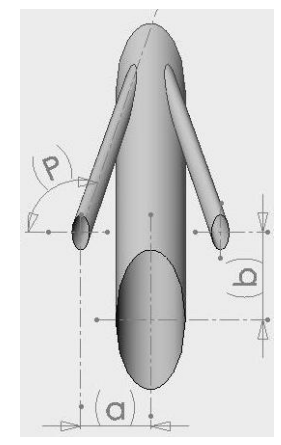

Top View

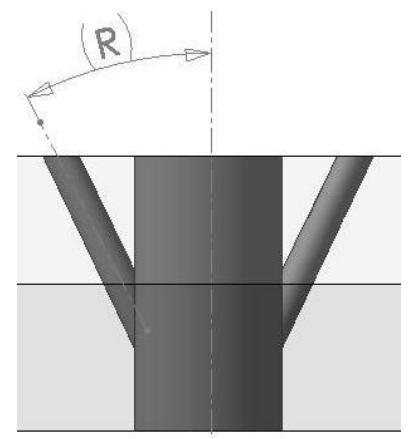

Front View

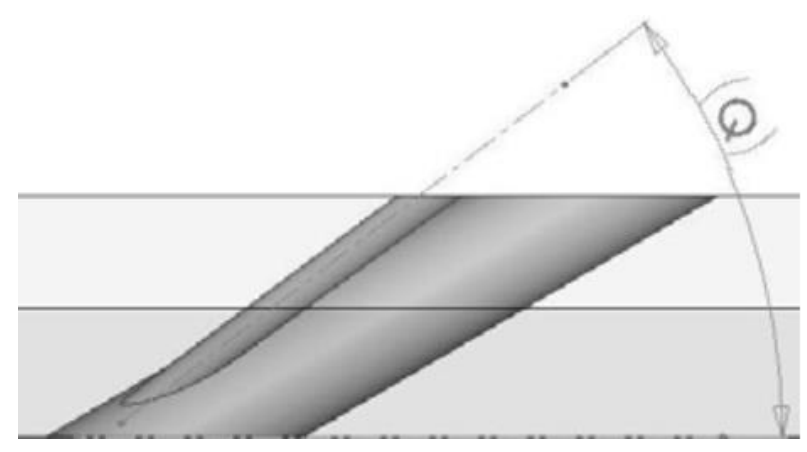

Side View

Figure 12: Three View Drawing of Anti-Vortex Hole [13]

Dhungel et al. [13] experimentally examined six different alterations to the AVH secondary holes for low turbulence cases to determine span-averaged film cooling effectiveness and span-averaged heat transfer coefficient shown in Figure 13 with main hole $\mathrm{P} / \mathrm{D}=3$. From this experimentation they discovered that the top three performing geometries were cases 3-5. Although case 5 was similar in performance there are concerns of structural integrity due to the minute amount of material between the secondary holes and the main hole. Cases 4 and 5 slightly outperformed case 3 at higher blowing ratios, and for these reasons case 4 will be studied in the current work. 


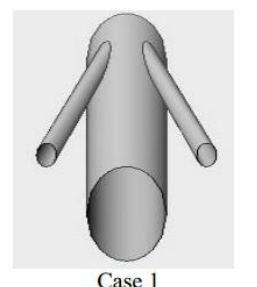

Case 1

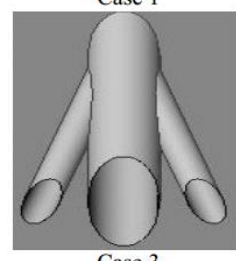

Case 3

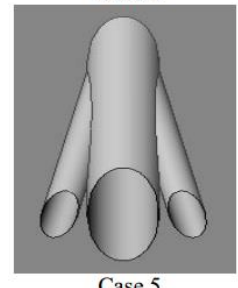

Case 5

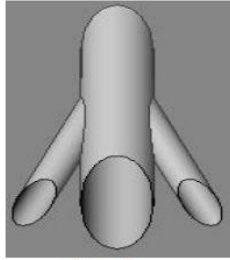

Case 2

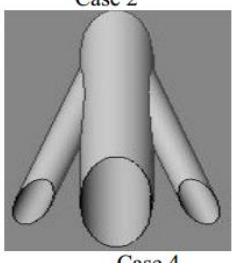

Case 4

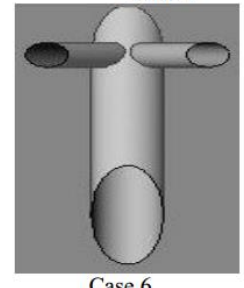

Case 6

Table 1: Experimentally Investigated AVH Geometry Parameters [13]

\begin{tabular}{|l|l|l|l|l|l|l|}
\hline & Case 1 & Case 2 & Case 3 & Case 4 & Case 5 & Case 6 \\
\hline $\mathrm{D}(\mathrm{cm})$ & 0.32 & 0.63 & 0.63 & 0.63 & 0.63 & 0.63 \\
\hline $\mathrm{D}(\mathrm{cm})$ & 1.27 & 1.27 & 1.27 & 1.27 & 1.27 & 1.27 \\
\hline $\mathrm{a}$ & 1.27 & 1.42 & 1.42 & 1.42 & 1.12 & 1.4 \\
\hline $\mathrm{b}$ & 1.6 & 0 & 0 & -0.56 & 0 & 4.12 \\
\hline $\mathrm{P}$ & 109.16 & 110.95 & 106.56 & 105.67 & 101.56 & 0 \\
\hline $\mathrm{Q}$ & 36.76 & 30 & 30 & 26.41 & 30 & 90 \\
\hline $\mathrm{R}$ & 24.95 & 34.74 & 27.21 & 27.91 & 19.43 & 30.62 \\
\hline $\begin{array}{l}\text { Start of } \\
\text { anti } \\
\begin{array}{l}\text { vortex } \\
\text { holes }\end{array}\end{array}$ & $\begin{array}{l}\text { Lower } \\
\text { end of } \\
\text { main } \\
\text { hole }\end{array}$ & $\begin{array}{l}\text { Mid } \\
\text { of } \\
\text { main } \\
\text { hole }\end{array}$ & $\begin{array}{l}\text { Lower } \\
\text { end of } \\
\text { main } \\
\text { hole }\end{array}$ & $\begin{array}{l}\text { Lower } \\
\text { end of } \\
\text { main } \\
\text { hole }\end{array}$ & $\begin{array}{l}\text { Lower } \\
\text { end of } \\
\text { main } \\
\text { hole }\end{array}$ & $\begin{array}{l}\text { Lower } \\
\text { end of } \\
\text { main } \\
\text { hole }\end{array}$ \\
\hline
\end{tabular}

Figure 13: Experimentally Investigated AVH Geometry Configurations [13]

Leblanc et al. [35] investigated numerically and experimentally another derivative of the AVH design, also referred to as the tripod design, where the secondary holes were of equal diameter to the main hole also requiring the minimum $\mathrm{P} / \mathrm{D}$ to be equal to 6 . $\mathrm{He}$ compared the cylindrical hole, $\mathrm{AVH}$, and $\mathrm{AVH}$ with trench geometries for low turbulence cases at density ratios of 0.95 and 1.45 for blowing ratios of $0.5-4.0$. The tripod design required $50 \%$ less air than the cylindrical hole, and provided on the order of 30-40\% overall improvement in film cooling effectiveness over the cylindrical hole. The tripod hole with a trench provided higher effectiveness in the trench than the other two designs, but had decreased effectiveness downstream relative to the tripod without a trench although still outperforming the cylindrical hole.

Recently at WVU, numerical investigations using the Reynolds Averaged Navier Stokes (RANS) and Unsteady Reynolds Averaged Navier Stokes (URANS) analyses have been completed by Repko et al. [36] and Repko et al. [37] for the AVH hole geometry in 
the current experimental work. In their work, varying non-dimensional freestream turbulence length scale based on main hole diameter $(\Lambda / D)$ and freestream turbulence intensity were studied to determine the impact of both parameters on film cooling effectiveness and heat transfer coefficient for a blowing ratio of 2.0. It was found that an increased length scale led to slightly higher centerline and span-averaged film cooling effectiveness, but was most noticeable for the $20 \%$ freestream turbulence intensity cases. Moreover, it was realized that increasing the freestream turbulence intensity also increased the film cooling effectiveness both on the centerline and span-averaged.

\subsection{CONTRIBUTIONS OF THE CURRENT STUDY}

The current work is aimed at determining experimentally the effects of elevated freestream turbulence at various blowing ratios upon an AVH configuration, accompanied by the reporting of centerline film cooling effectiveness and the convective heat transfer coefficient that had not been reported in prior studies. Previous experimental studies had also not investigated freestream turbulence above $\approx 1 \%$ for the AVH geometry, as a major concern with the AVH will be how the secondary jets interact with the main coolant jet in counteracting the CRV pair. This will be the first experimental facility to study the effects of higher freestream turbulence levels on an AVH geometry. 


\section{CHAPTER 2: EXPERIMENTAL SETUP}

\subsection{DESIGN, FABRICATION, AND LAYOUT OF EXPERIMENTAL FACILITY}

\subsubsection{FREESTREAM SECTION}

The experimental film cooling facility at West Virginia University, shown in Figure 14 and Figure 15, is an open loop tunnel that has been designed and fabricated by the author to non-dimensionally simulate the aerothermal environment experienced by the first stage vane of a gas turbine engine as a flat plate representation. The freestream flow is provided by a Cincinnati Radial Blade Exhauster (RBE) fan on a direct drive motor/shaft configuration driven by a $7.5 \mathrm{hp}$ Baldor motor. The RBE intake is fitted with a fine paper screen to prevent minute particles from encroaching into the freestream flow and collecting on the mesh screen heaters as will be described later. The motor at full load is capable of 3625 RPM resulting in tunnel freestream velocities of approximately $25 \mathrm{~m} / \mathrm{s}$. The motor is controlled by a Bardac Optidrive E2 controller allowing for variable freestream velocities.

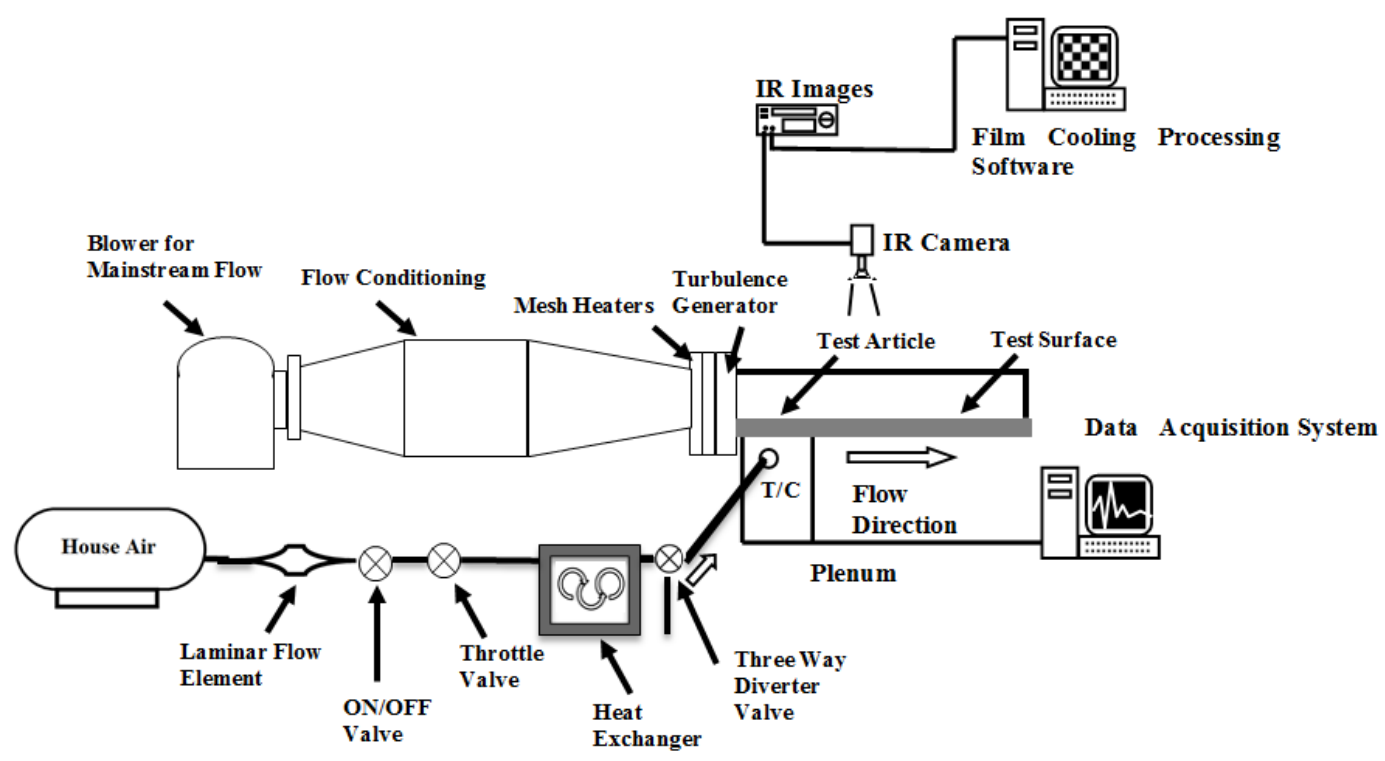

Figure 14: WVU Open Loop Tunnel Schematic 


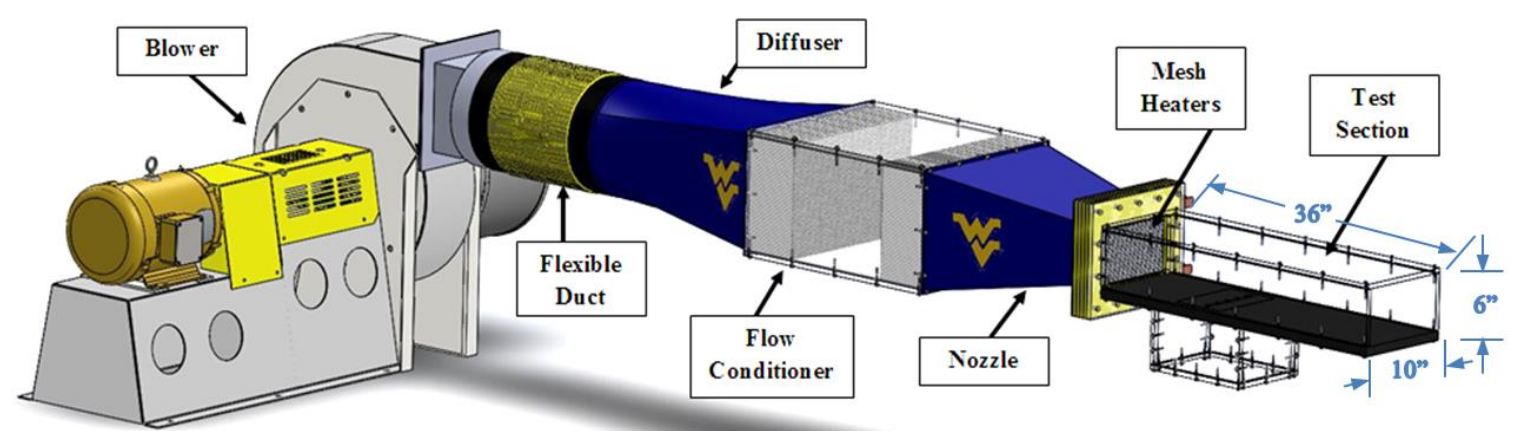

Figure 15: SolidWorks Model of Wind Tunnel Freestream Loop

The flow passes through a flexible duct to reduce any vibrations created by the blower/motor configuration. The flow is then ducted through a diffuser which slows the velocity of the freestream as well as aids in creating a uniform cross-sectional velocity. The diffuser also converts the flow from a circular cross-section to a larger rectangular crosssection which will be important for the stages downstream. The cross-sectional area ratio of expansion is 2:1 and the diffuser is designed with an expansion angle as small as possible in an attempt to prevent boundary layer separation [38].

Upon exiting the diffuser, flow enters a conditioning/straightener section comprised of two Plascore honeycomb sections to eliminate the swirl in the tunnel from the blower upstream, as well as provide a nearly laminar flow at the entrance of the nozzle. The nozzle has a cross-sectional area reduction of $3.6: 1$ providing an acceleration of the freestream while maintaining a rectangular cross-section. The flow is conditioned further as it passes through two 304 stainless steel wire mesh screen resistance heaters connected in series so as to reduce the current requirements on the welder used to provide current. The screens provide an air temperature step change and additional reductions in flow velocity variation. The mesh is a $200 \times 200$ wire per square inch arrangement with wire diameter of 0.0016 inches and a spacing of 0.0034 inches between wires resulting in an open area of $46 \%$ for 
each screen. Other mesh sizes were evaluated in conjunction with this mesh size, but were not used because they did not produce the step temperature change desired or obstructed the freestream flow beyond that desired.

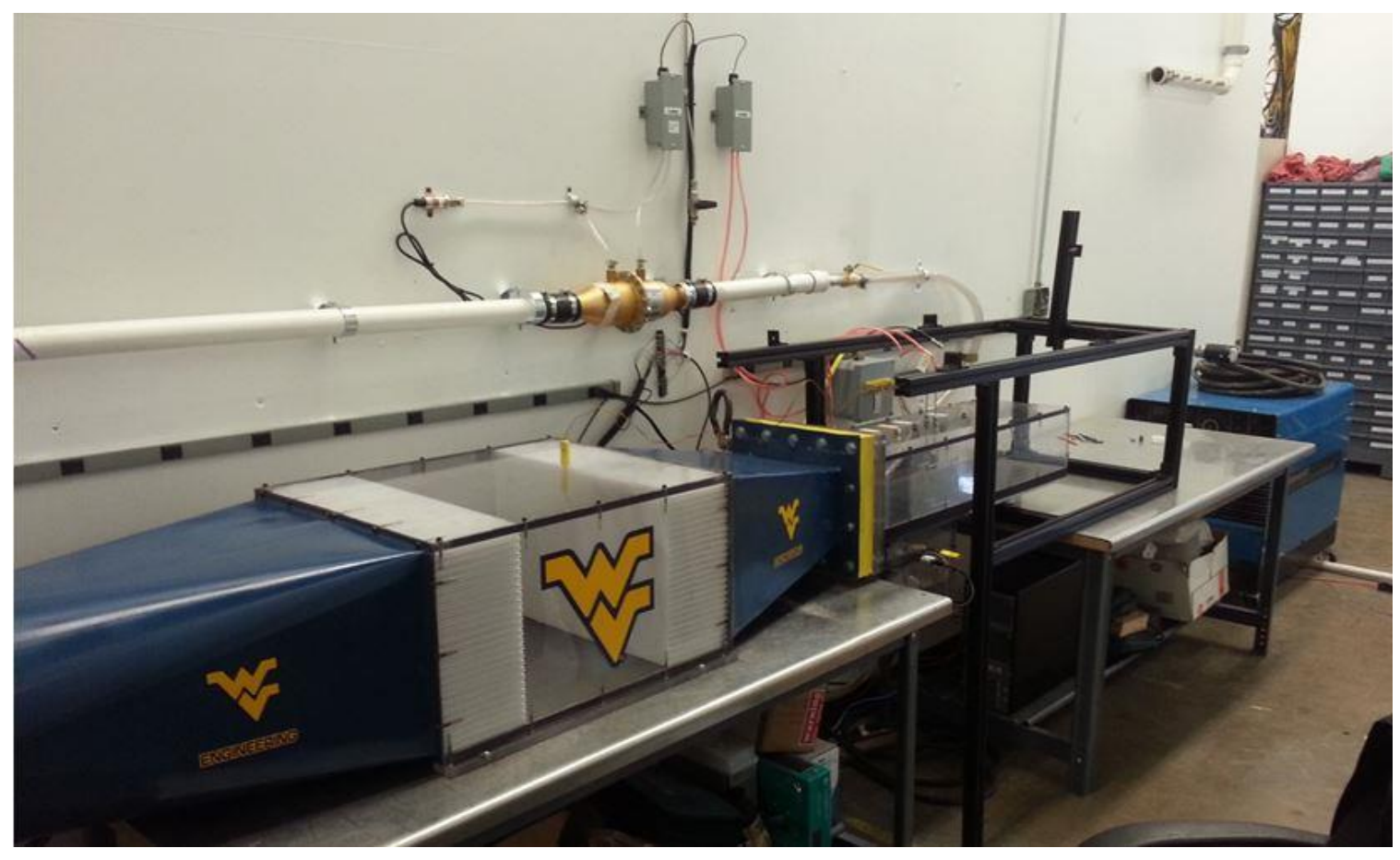

Figure 16: Wind Tunnel Facility during Probe Traverse

Figure 16 displays the freestream section of the current facility. The mesh heater is controlled by a constant voltage Miller Deltaweld 451 MIG welder connected to copper bus bars transferring the electrical connection to the mesh. The bus bars and mesh are insulated with GP03 electrical grade fiberglass capable of withstanding maximum temperatures of $265^{\circ} \mathrm{F}\left(130{ }^{\circ} \mathrm{C}\right)$ while being arc resistant, and are of high strength for structural robustness. The mesh heaters are capable of a step temperature increase of approximately $10^{\circ} \mathrm{C}$ at the current Reynolds number based on cooling hole diameter, as described later, of approximately 10,000. Performing a thermocouple traverse showed that the freestream flow was nearly uniform in temperature after passing through the heaters. Once the flow has been conditioned to a near uniform and laminar state it passes through 
the turbulence grid section to achieve the desired freestream conditions. The three current options for turbulence intensities are 1\% (no grid), 7.5\% (small bar grid), and 11.7\% (large bar grid) at the leading edge of the coolant holes. The turbulence grids will be discussed in the following section.

\subsubsection{TURBULENCE GRIDS}

In order to generate various levels of freestream turbulence intensities, two passive lattice style turbulence grids were designed based on research conducted by Baines and Peterson [39]. Figure 17 illustrates how the grid can be imagined to produce a series of jets in each opening between the bars that spread and coalesce downstream with other jets resulting in turbulent eddies produced from zones of shear around each jet.

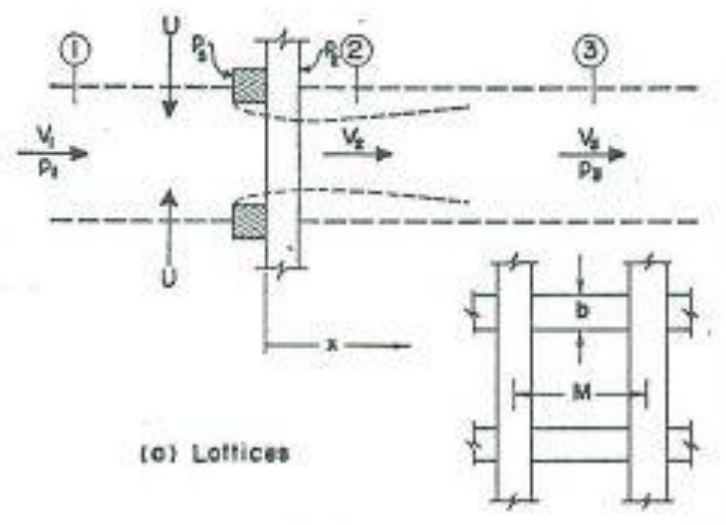

Figure 17: Freestream Flow through Turbulence Grid [39]

Theoretical decay of turbulence and theoretical turbulence length scale allowed for accurate prediction of grid performance in the tunnel. A MATLAB ${ }^{\circledR}$ program was developed to allow for calculations of these parameters so that a small range of sizes could be implemented for testing to reduce the amount unnecessary grids produced, with the results being shown in Figure 18. Theoretical decay of turbulence and length scale are both a function of the normalized streamwise distance according to equations (2-1) and (2-2), 
where $(\mathrm{Tu})$ is the freestream turbulence intensity, $(\Lambda)$ is the streamwise turbulence length scale and $(\mathrm{x})$ is the streamwise distance:

$$
\begin{aligned}
& \mathrm{Tu}_{\mathrm{x}}=\mathrm{c}\left(\frac{\mathrm{x}}{\mathrm{b}}\right)^{\mathrm{n}} \\
& \Lambda_{\mathrm{x}}=\mathrm{ab}\left(\frac{\mathrm{x}}{\mathrm{b}}\right)^{\mathrm{m}}
\end{aligned}
$$

The constants (c) and (a) are given as 1.12 and 0.20 , respectively, and (b) is the known bar diameter in inches in Figure 18. The exponents (n) and (m) are also given values, equal to 0.53 and $-5 / 7$ respectively. Shortly after exiting the grid the flow is anisotropicly turbulent while entering the establishment region where the flow approaches isotropic turbulence. In the region that the flow is anisotropicly turbulent, vortex shedding from the bars decomposes into turbulent eddies and that develop into nearly isotropic turbulence further downstream. As the flow moves away from the grid the freestream turbulence intensity decays as the length scale of the turbulent eddies increase. This may be easily visualized in Figure 18. 


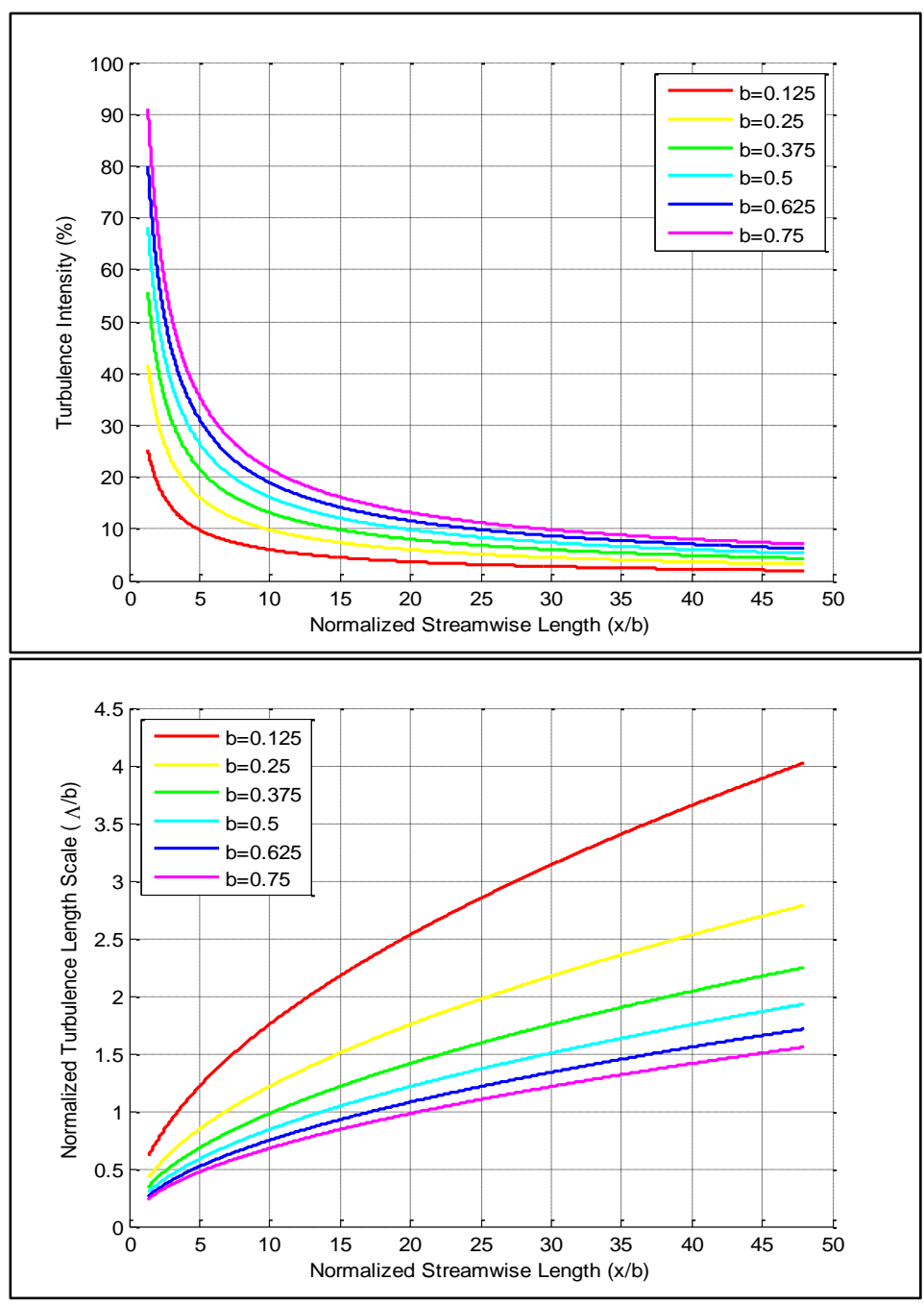

Figure 18: Non-Dimensionalized Theoretical Turbulence Intensity and Length Scale for Mulitple Bar Diameters

From these results a $1 / 2$ " and a $1 / 4$ " grid were constructed (Figure 19) and evaluated with the use of a one dimensional hotwire anemometer to evaluate turbulence intensity and turbulence length scale for each grid at multiple streamwise locations in the facility. Baines and Peterson [39] found through experimentation that nearly isotropic flow can safely be assumed 5 to 10 bar diameters downstream; thus the coolant hole locations were placed well beyond 10 bar diameters as specified. The $1 / 4$ " grid produced a freestream turbulence intensity of $7.5 \%$ at the location of the leading edge of the coolant holes with a nondimensional length scale based on hole diameter $\left(\Lambda_{\mathrm{x}} / \mathrm{D}\right)$ of 2.24 . The $1 / 2$ " grid produced a 
turbulence intensity of $11.7 \%$ with length scale of 1.4 at the hole leading edge. Without the turbulence grids the flow has a turbulence intensity of $1 \%$ with a non-dimensional length scale of 6.4 at the leading edge of the coolant holes.
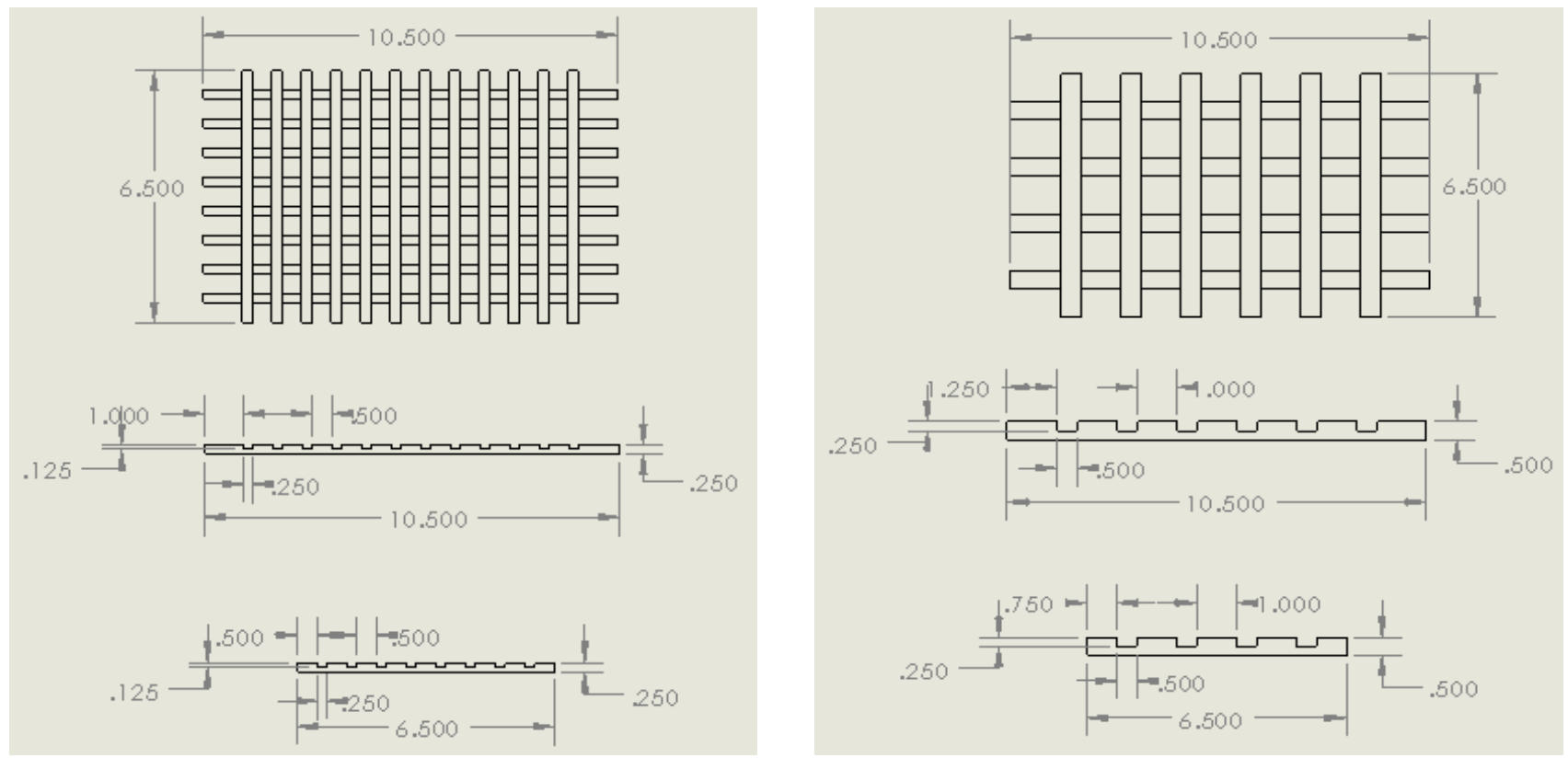

Figure 19: 1/4" and 1/2" Turbulence Grids

Figure 20 and Figure 21 display the freestream turbulence intensity and nondimensionalized freestream turbulence length scale of experimental trials compared to those predicted numerically using the theory of Baines and Peterson at nondimensionalized distances downstream for a grid bar diameter of $1 / 4$ ". 


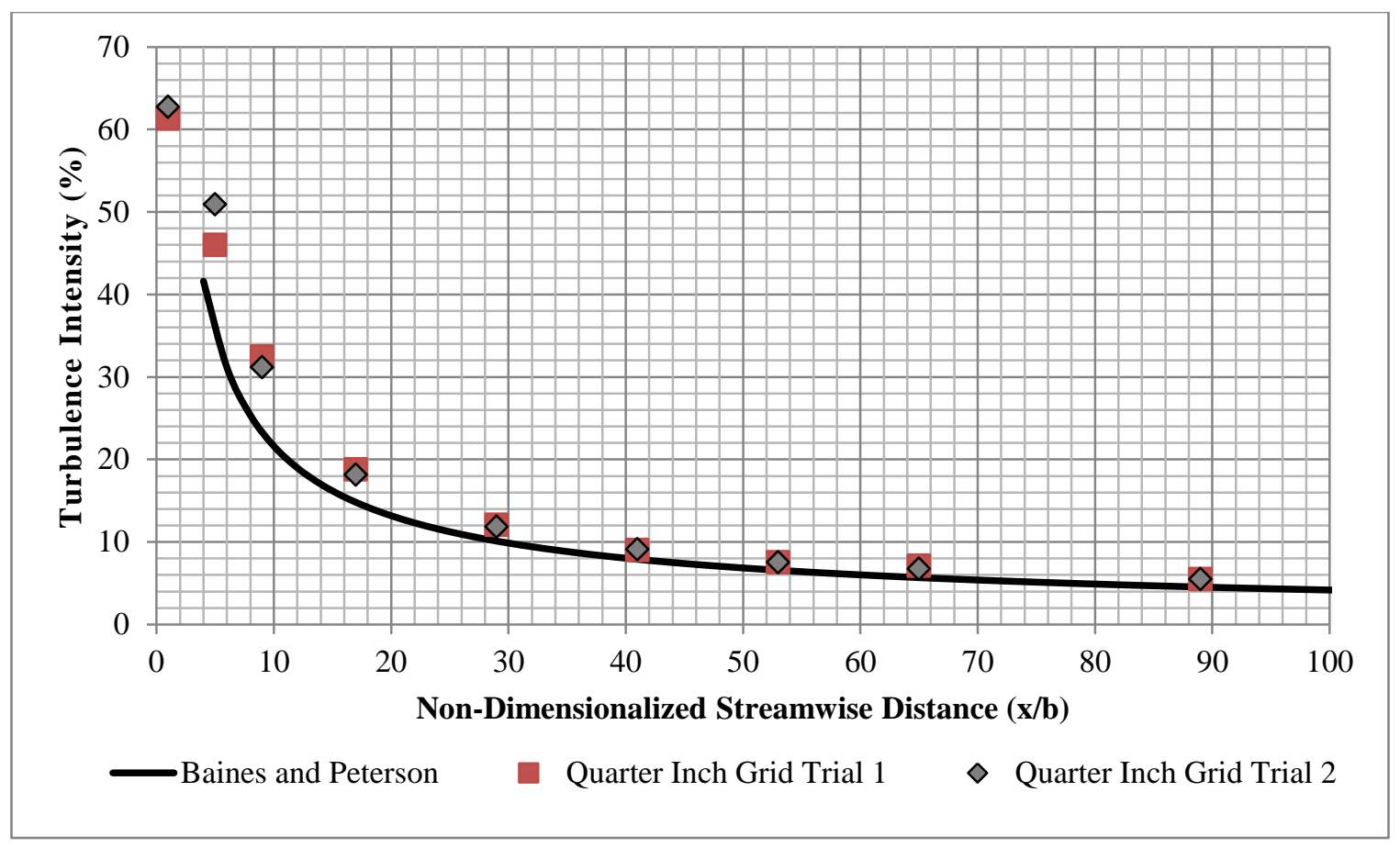

Figure 20: Turbulence Intensity Decay for 1/4" Grid

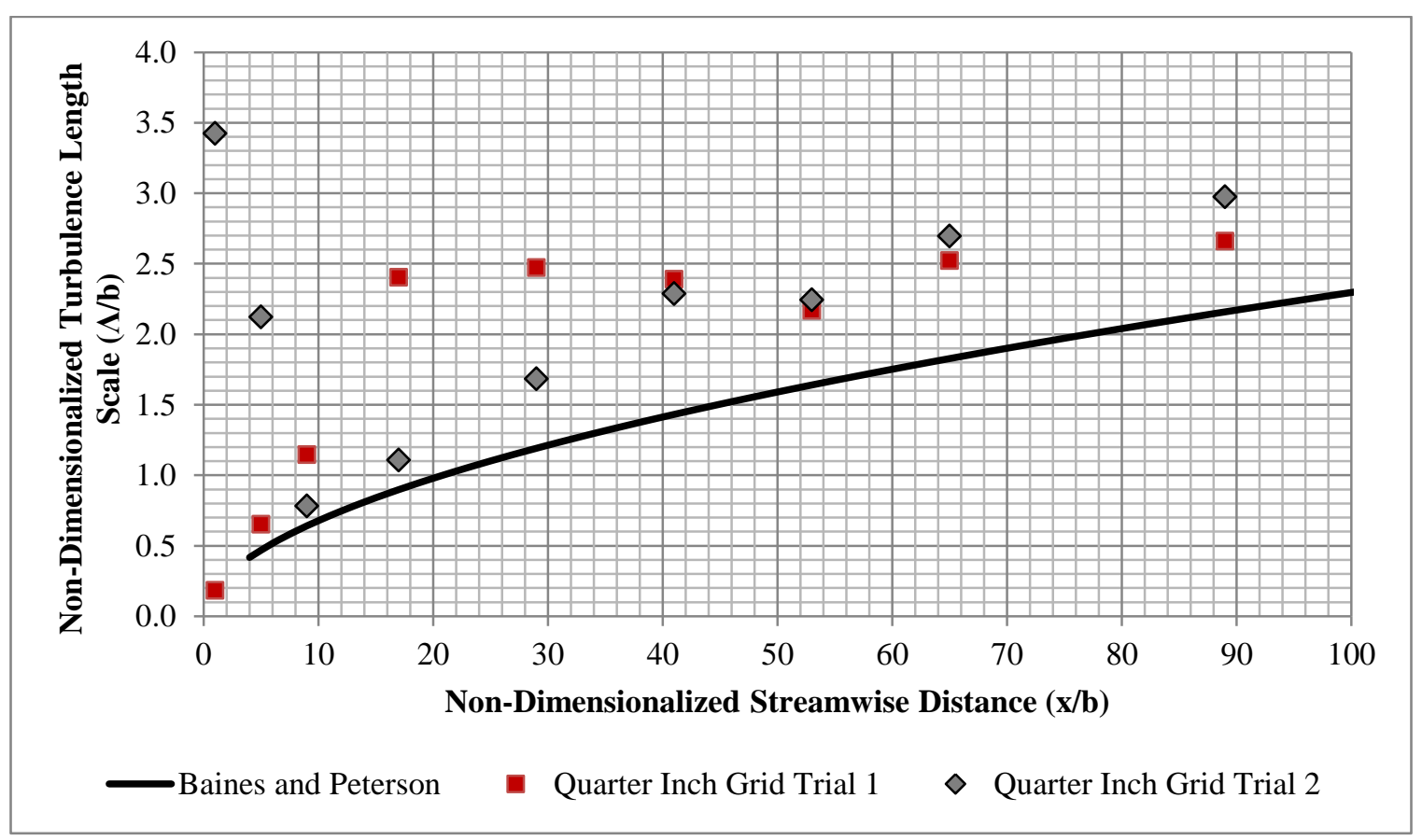

Figure 21: Growth of Non-Dimensionalized Turbulence Length Scale for 1/4" Grid

The freestream turbulence decays almost as predicted by the Baines and Peterson correlation and virtually predicts the turbulence intensity downstream where the leading 
edge of the coolant holes resides. However, the turbulence length scale is underpredicted using the Baines and Peterson correlation.

Figure 22 and Figure 23 display the freestream turbulence intensity and nondimensionalized freestream turbulence length scale of experimental trials compared to those predicted numerically using the theory of Baines and Peterson at nondimensionalized distances downstream for a bar diameter of $1 / 2$ ". As was the case with the $1 / 4$ " diameter bar grid, the numerically predicted turbulence intensities were not very accurate in the anisotropic turbulent region residing in the first ten bar diameters downstream, but accurately predicts the turbulence intensity at the leading edge of the cooling holes $(\approx 25$ bar diameters for $1 / 2 "$ grid and $\approx 50$ bar diameters for $1 / 4$ " grid). Contrary to the smaller grid, the $1 / 2$ " bar diameter grid non-dimensionalized turbulent length scale was accurately predicted and resided directly between the experimental cases directly after the anisotropicly turbulent region.

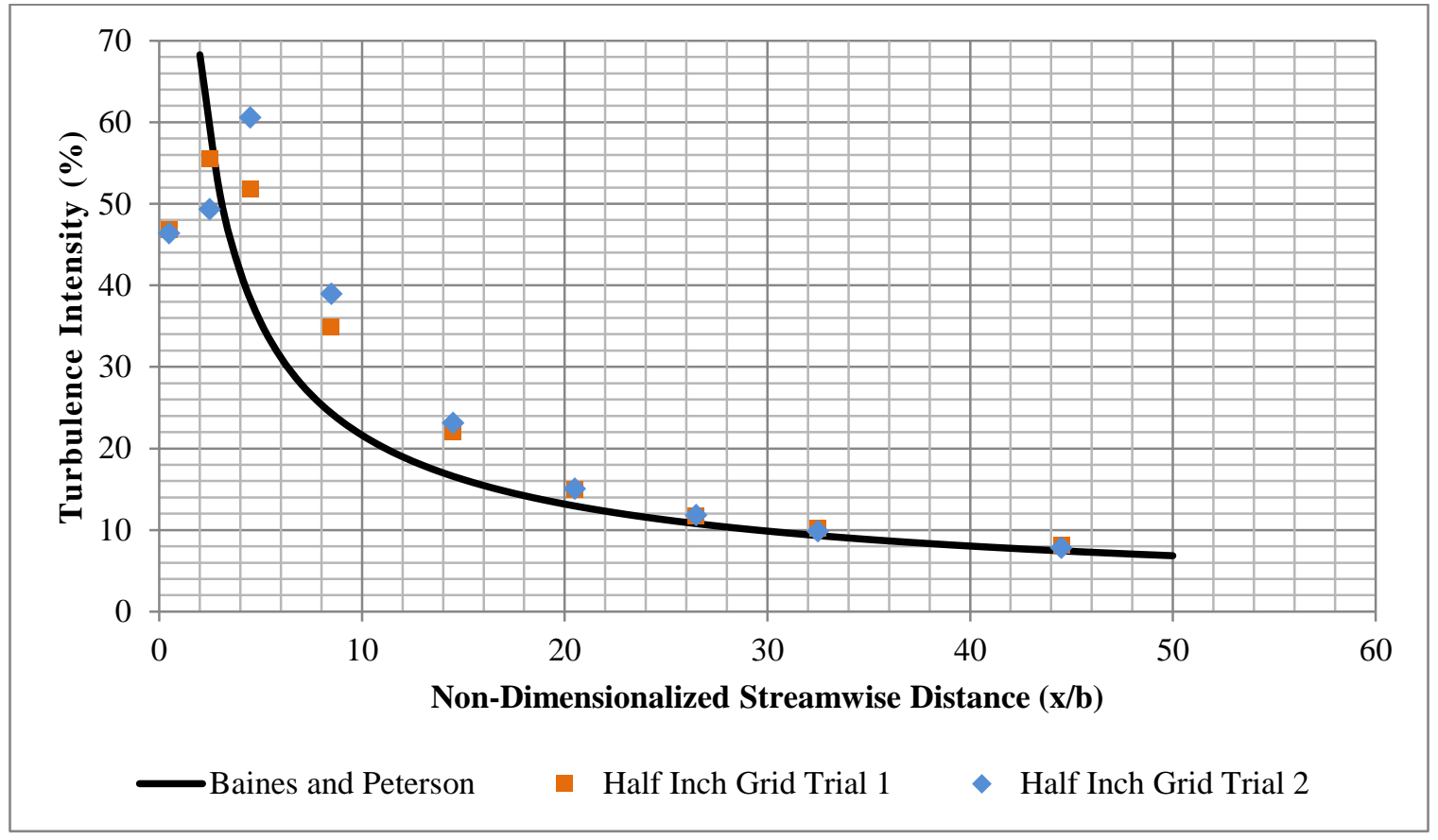

Figure 22: Turbulence Intensity Decay for 1/2" Grid 


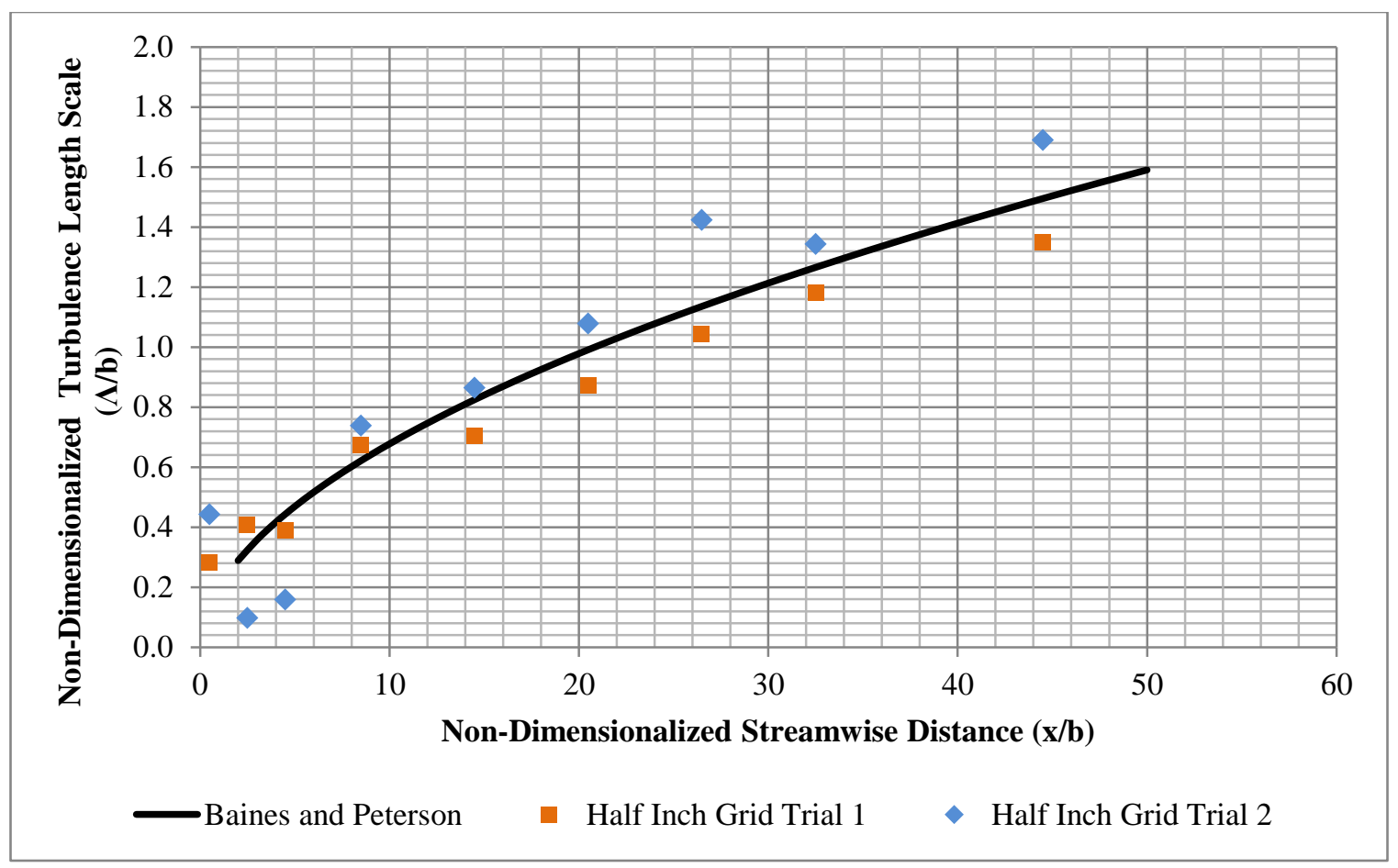

Figure 23: Growth of Non-Dimensionalized Turbulence Length Scale for 1/2" Grid

\subsubsection{TEST SECTION}

The test section in the current facility represents the near hole region on the pressure and suction surfaces on turbine airfoils. Pictured in Figure 24 is a film cooled first stage vane of a gas turbine engine. On the right is a zoomed in picture highlighting the area of interest on the pressure surface, which is represented by the test section in the current facility with the freestream flow traveling from left to right.

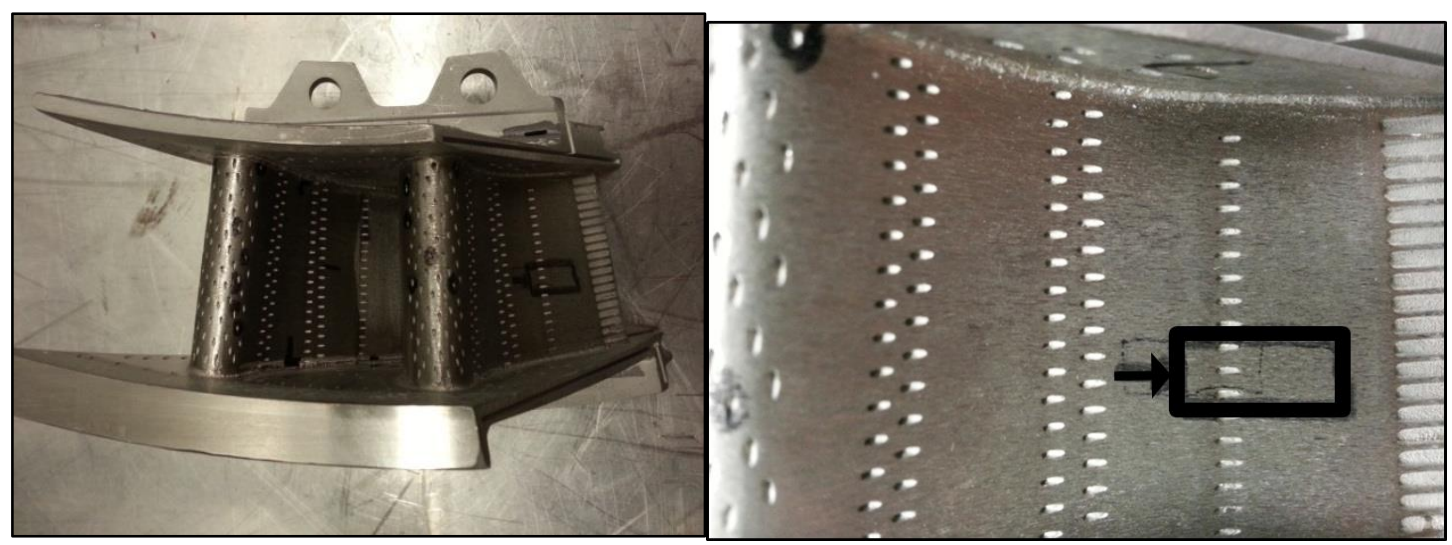

Figure 24: First Stage Gas Turbine Vane with Test Section Region of Interest 
The test section of the new flat plate film cooling wind tunnel presented in Figure 25 is 6 inches tall by 10 inches wide by 36 inches long. There are currently two different "lids" (top of test section) which may be used for different measurements that can be easily interchanged. The test section is comprised of $1 / 2$ inch Lexan side walls as well as two different $1 \frac{1}{2}$ inch Lexan lids.

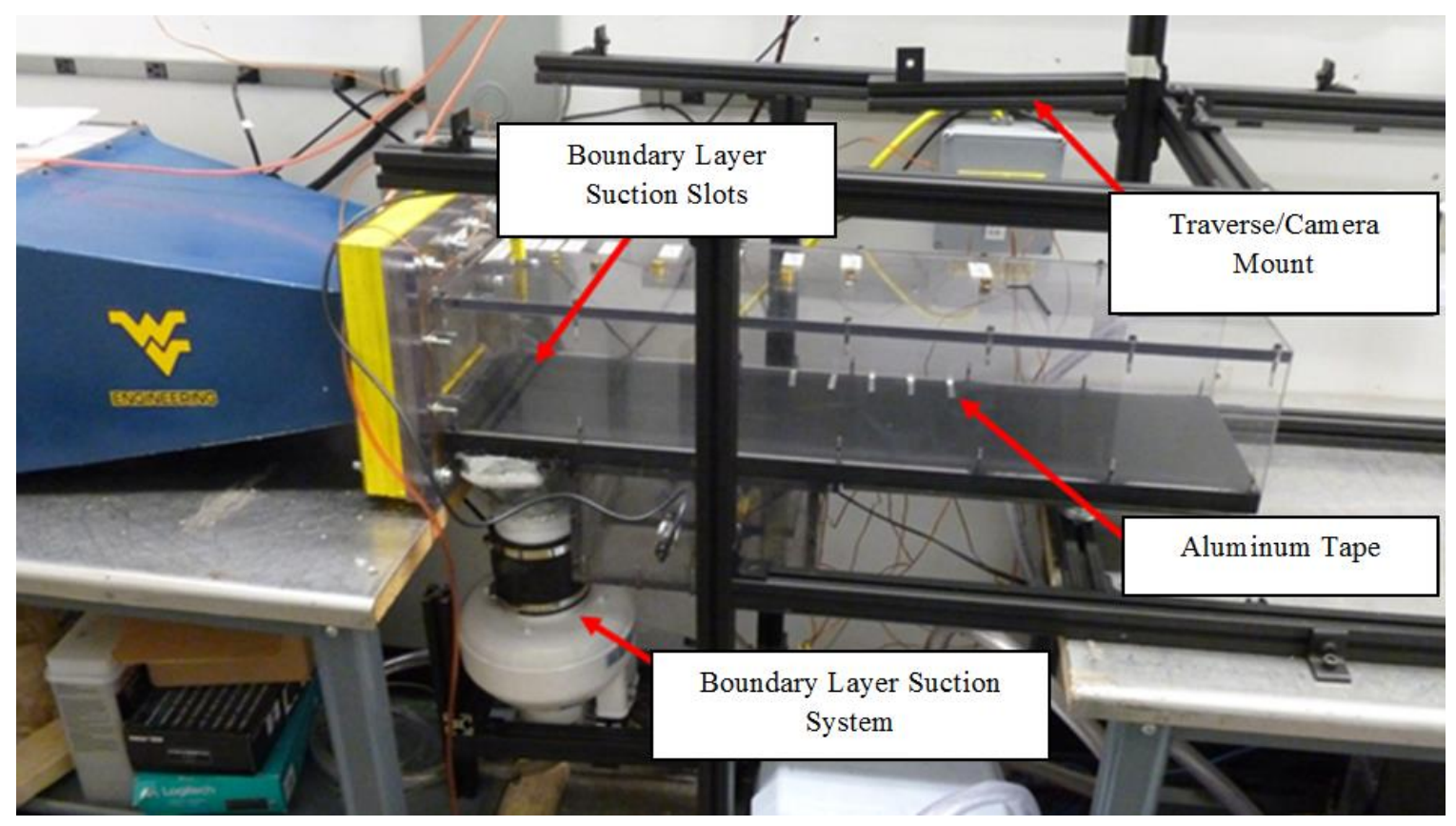

Figure 25: Test Section with Boundary Layer Suction System

The first lid is entirely solid, but is fitted with nine tapped holes for hotwire, pitot-static and boundary layer measurements which were performed along the length of the test section. The second lid has a slot that has been milled out and replaced by a polyethylene window for the IR testing. The base of the test section presented in Figure 26 is made of one inch thick machinable black ABS plastic due to its low thermal conductivity. Aluminum tape was applied outside of the region of interest at known streamwise distances after the cooling holes due to their largely different emissivity from the rest. These pieces of tape were using during infrared camera operation as distance references along the tunnel. 


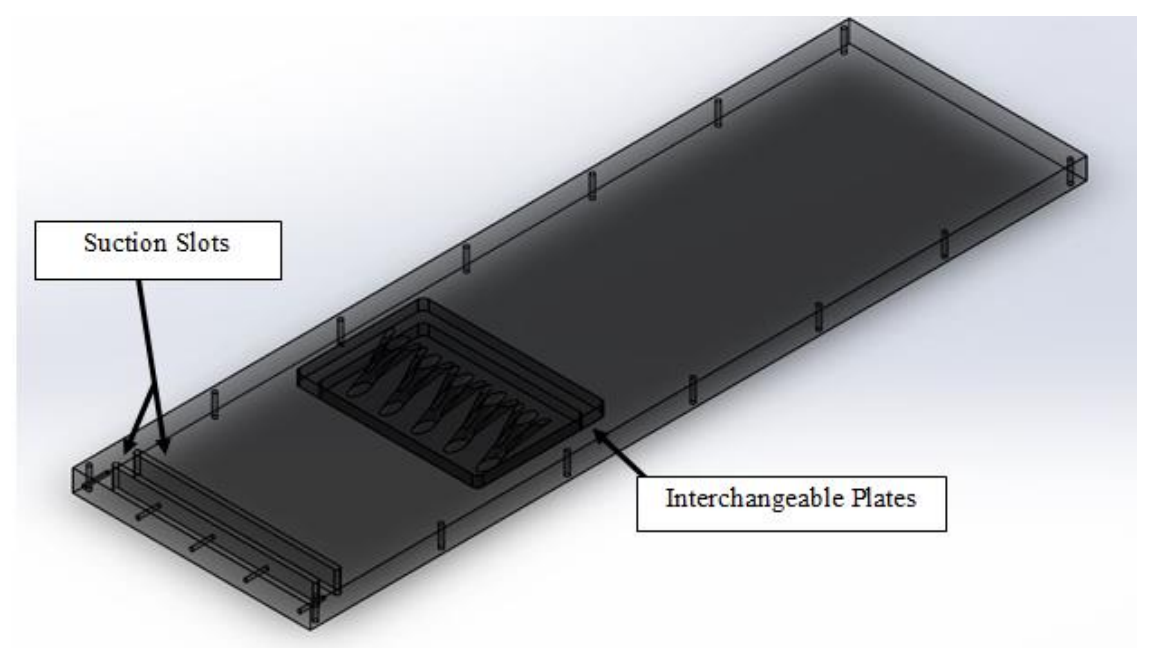

Figure 26: Test Section Base Shown with Interchangeable Plate Insert

Another key feature of the test section base is the suction slots located at the beginning of the test section to control the boundary layer thickness. The trailing edge of the second slot is 19 hole diameters upstream of the leading edge of the cooling holes and was connected to a RadonAway RP145 radon mitigation fan to provide suction. Based on the work of Radomsky and Thole [40] and Jenkins, Varadarajan, and Bunker [41] a nondimensional boundary layer height $(\delta / D)$ based on main cooling hole diameter was determined for both suction and pressure surfaces of an airfoil. The previous work found the non-dimensional boundary layer height for low turbulence cases to be 0.15 to 1.4 and for high turbulence cases becomes 0.17 to 3.74 , resulting in the target ranges for the current facility representing the range of boundary layer thickness of the pressure and suction surfaces of various locations of a turbine airfoil. Conducting traverses with a total pressure boundary layer probe just before the leading edge of the coolant holes, a non-dimensional boundary layer of 0.5 was found with the suction system on; this value is safely between the given ranges.

Several fine increment pitot-static probe traverses were conducted vertically at multiple locations along the test section to determine the velocity profile of the freestream. Figure 
27 shows the traverse in use and a hotwire anemometer being calibrated with a pitot-static probe. Utilizing these velocity profiles, a mass flow rate could be determined from the known tunnel width, vertical height between measurements, and density of the air. To achieve a more accurate mass flow rate a horizontal traverse would have been beneficial, but was not possible with the current instrumentation without approaching through both sides of the tunnel for one traverse.

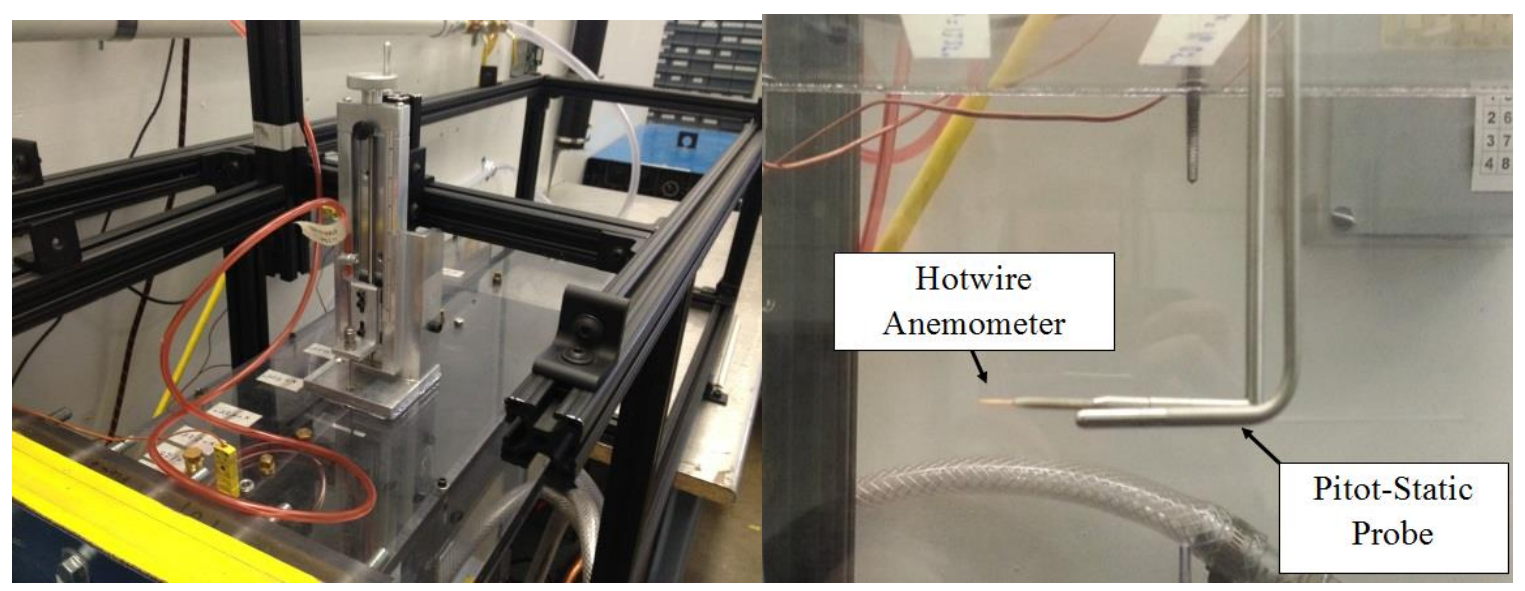

Figure 27: Traverse System (Left), Pitot-Static and Hotwire Probes (Right)

\subsubsection{COOLANT LOOP}

Air is supplied by an in-house low pressure compression system and is controlled by an ON/OFF valve and throttle valve to adjust the coolant flow rate to achieve variable blowing ratios. Air is metered by a Meriam 50MC2 laminar flow element (LFE) which has multiple laminar channels created by the matrix section, as shown in Figure 28, and the air temperature is measured by a thermocouple upstream. As the air flows through the passageways in the matrix of the LFE the friction of the air on the walls of the channels creates a pressure drop which is measured by a differential pressure transducer. A calibration is performed at the Meriam facility for the LFE resulting in a specific 
calibration curve with known constants B and C which can be applied to equation (2-3) to find the standard volumetric flow rate of the coolant flow.

$$
\mathrm{SCFM}=\left(\mathrm{B} * \mathrm{DP}+\mathrm{C} * \mathrm{DP}^{2}\right) *\left(\frac{\mu_{\mathrm{std}}}{\mu_{\mathrm{air}}}\right) *\left(\frac{\mathrm{T}_{\mathrm{std}}}{\mathrm{T}_{\mathrm{air}}}\right) *\left(\frac{\mathrm{P}_{\mathrm{air}}}{\mathrm{P}_{\mathrm{std}}}\right)
$$

Here DP is the measured differential pressure measured in inches of water column and the three ratios are related to the current air viscosity, temperature, and upstream pressure compared with standard air (std).

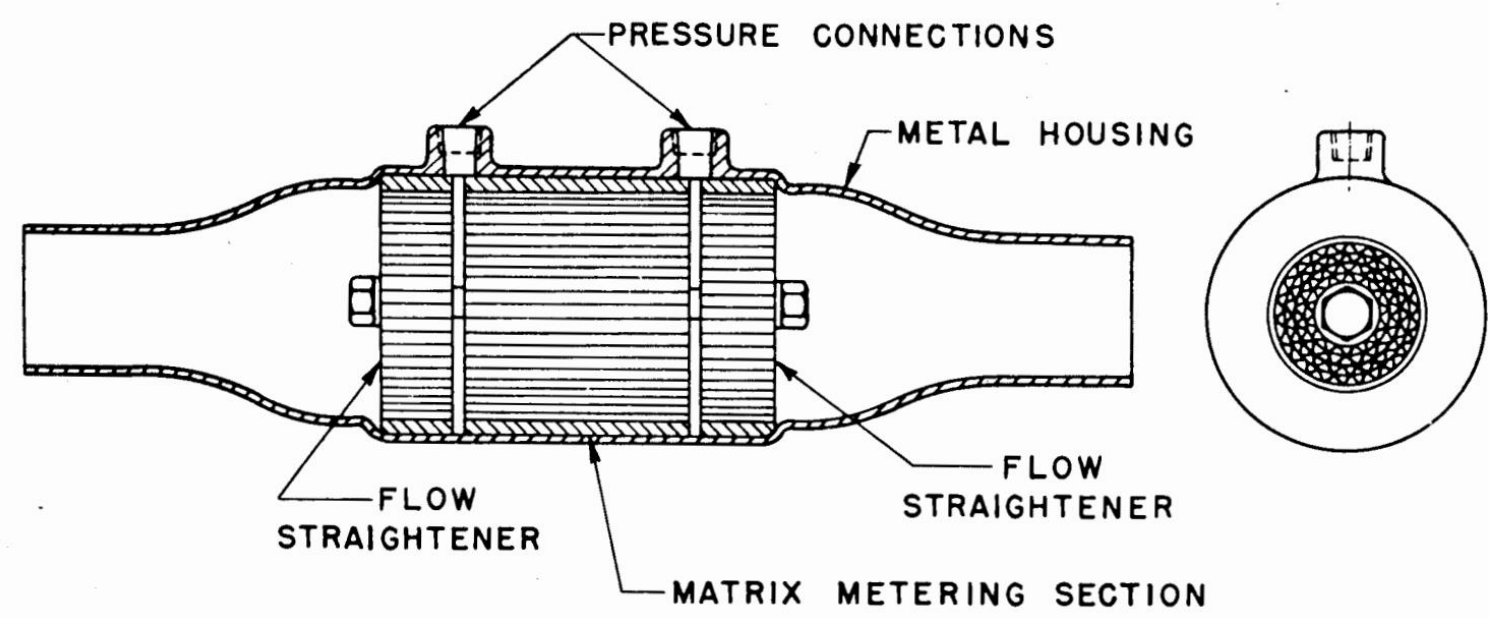

Figure 28: Laminar Flow Element [42]

Upon exiting the LFE the air is routed to a copper tube bank submerged in a dry ice and ethylene glycol bath reducing the air by $9^{\circ} \mathrm{C}$ and then to a 3-way ball valve to duct the air away from the plenum until the test is to be activated. Once routed towards the plenum the coolant air is split and routed to each side of the plenum shown in Figure 29. The two entrances into the plenum are aimed directly facing the other to have the jets impinge upon themselves to reduce the velocity of the air entering the coolant holes and ensure a more uniform distribution of flow. 


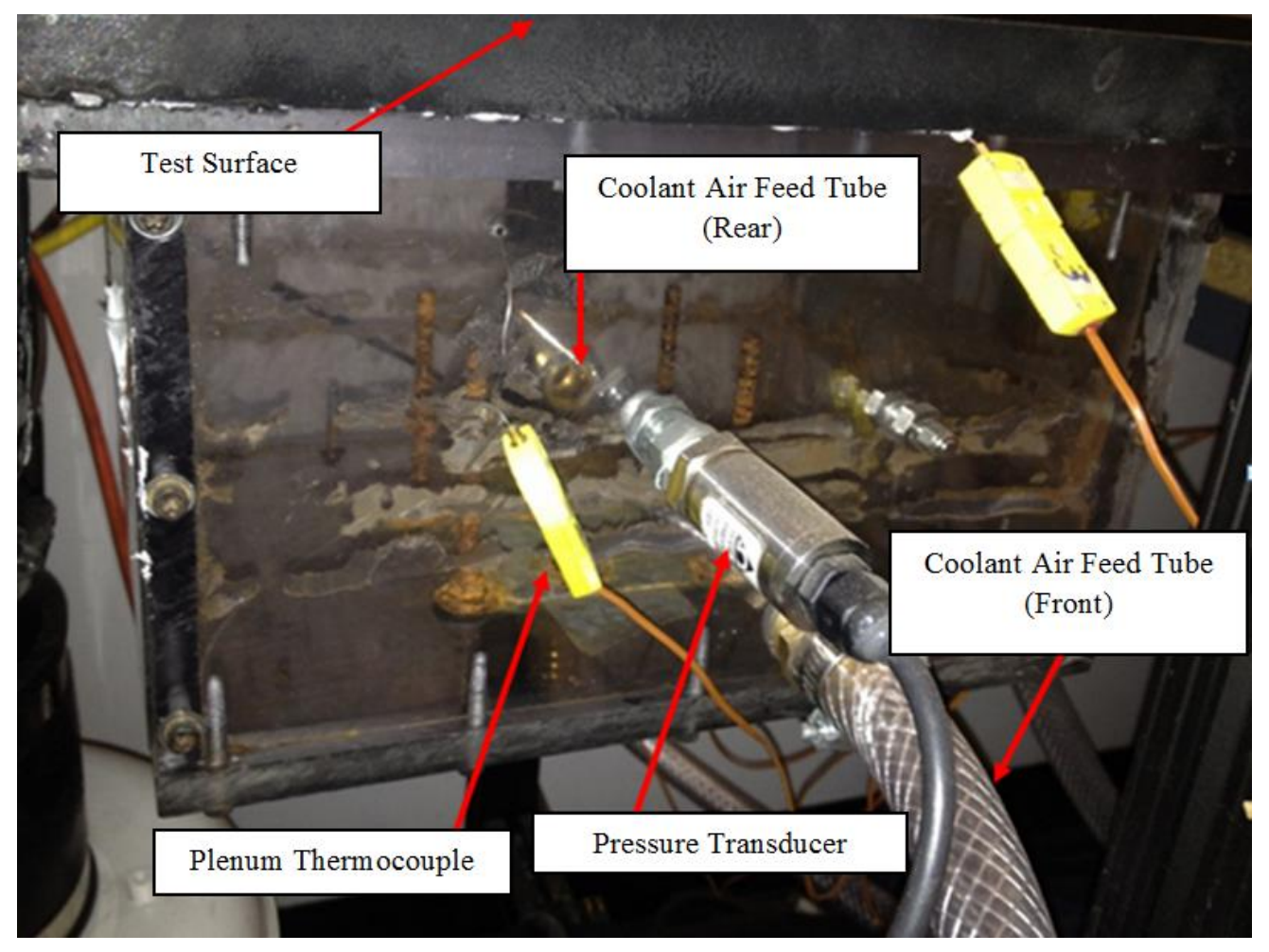

Figure 29: Plenum for Coolant Flow

A liquid nitrogen bath was initially used rather than the final ethylene glycol and dry ice bath, but had to be dismissed due to cost and problematic frost accumulation in the plenum and the feeding tubes. The achieved density ratio for testing conditions is 1.05 which is lower than the actual density ratio $(\approx 1.5)$ of that in an actual engine. An achieved temperature ratio $T_{o} / T_{c}$ of 1.06 was realized, but this also is lower than that for engine conditions which is approximately 1.4-1.6.

\subsubsection{PLATE INSERTS}

Figure 30 shows the isometric views of both the $30^{\circ}$ conventional straight hole used during the benchmarking phase, and the selected AVH geometry used during the experimental operation. The plates were developed in SolidWorks ${ }^{\circledR}$, a computer-aided design (CAD) software, to the specifications in the following paragraph. The plates were designed to be interchangeable by resting on a designed ledge around the perimeter of the 
plate. After the designed plates were printed in a Stratasys Dimension 1200es threedimensional printer out of the aforementioned black ABS plastic material, the plate was sealed around the underside using silicon and the top was sealed using wood putty with minor traces of acetone to faintly fuse the edges. Once the putty had been applied, the entire surface was sanded with multiple stages of fine grit sandpaper being used mounted to a large sanding block to ensure a truly flat surface. Finally, the surface was coated with a flat black paint for a uniform emissivity of the test surface. Swapping out the plates is a two-day process between disassembling the test section, inserting the new plate and preparing the surface with the above process, and reassembling the test section.

The main hole diameters of both plates are $1 / 2$ " with a hole pitch over diameter ratio (P/D) of 3 and a length over diameter ratio (L/D) of 4 while both sets of holes have an angle of inclination from the horizontal of $30^{\circ}$. The "metered" flow area remains the same between plates due to the inlet area of the coolant holes being identical resulting in identical mass flow rates although the exit areas of the coolant holes are rather different.

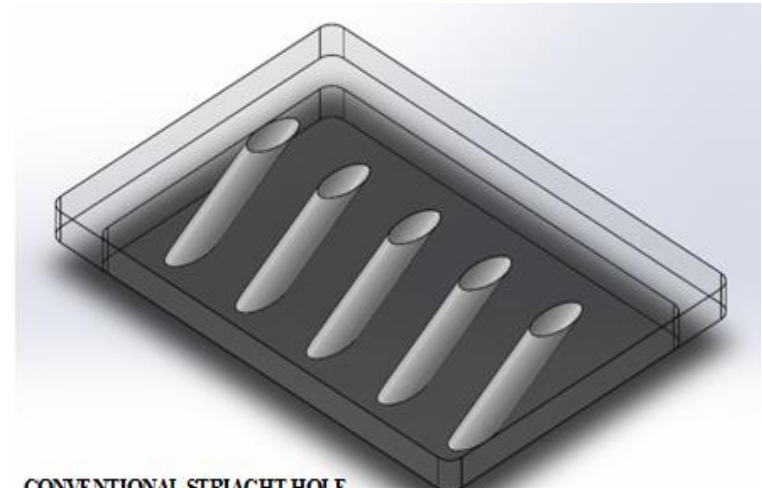

CONVENTIONAL STRIAGHT HOLE

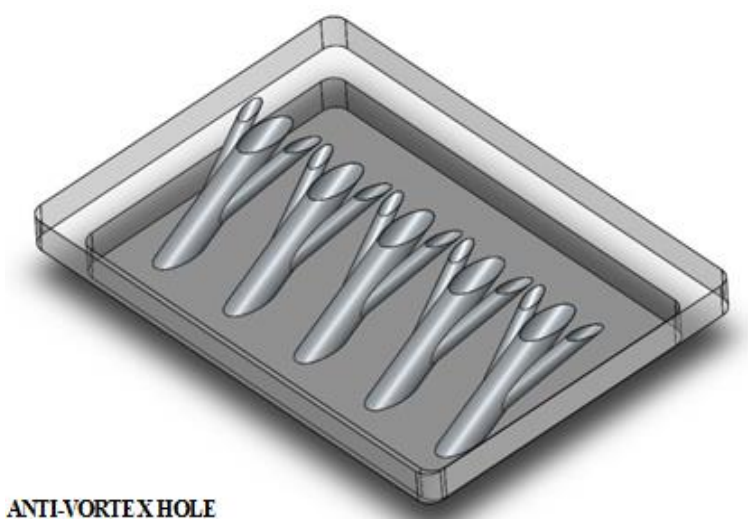

ANTI-VORTEX XOLE

Figure 30: Isometric Views of $3^{\circ}$ Conventional Straight Hole and Selected AVH Geometry 


\subsection{DATA ACQUISITION (DAQ) AND INSTRUMENTATION}

Data acquisition and instrumentation were used throughout the process of commissioning the tunnel, in order to characterize and benchmark the conditions of the tunnel, as well as during experimental testing. Key data acquired from the tunnel to measure film cooling effectiveness included, but was not limited to: freestream flow velocity, freestream turbulence intensity, freestream Reynolds number based on main cooling hole diameter, pressure loss through the tunnel, and increasing step temperature change with increasing voltage supplied by the welder.

\subsubsection{NATIONAL INSTRUMENTS DAQ}

All pressure, temperature, and hotwire measurement data is recorded through a series of in-house produced National Instruments LabVIEW ${ }^{\circledR}$ codes. The first code was used for hotwire measurements, and ran for ten seconds while recording data at $50 \mathrm{kHz}$ for a total of 500,000 time data points. The second code was used for thermocouple and pressure measurements during the transient heat transfer test. This was collected at $500 \mathrm{~Hz}$ for 30 seconds while outputting real time results to a screen to monitor sensor functionality.

In Figure 31 the four-slot, Ethernet connected, compact $9184 \mathrm{DAQ}$ is pictured with all four modules. The first module on the left is the four-channel NI 9215 BNC connection module used for hotwire measurements. The second and third modules are NI 9211 thermocouple modules with four-channels. The module on the right is also a four-channel NI 9215 module, but is a screw terminal connection for use with the pressure sensors. The pressure and temperature data are collected simultaneously during the transient test. 


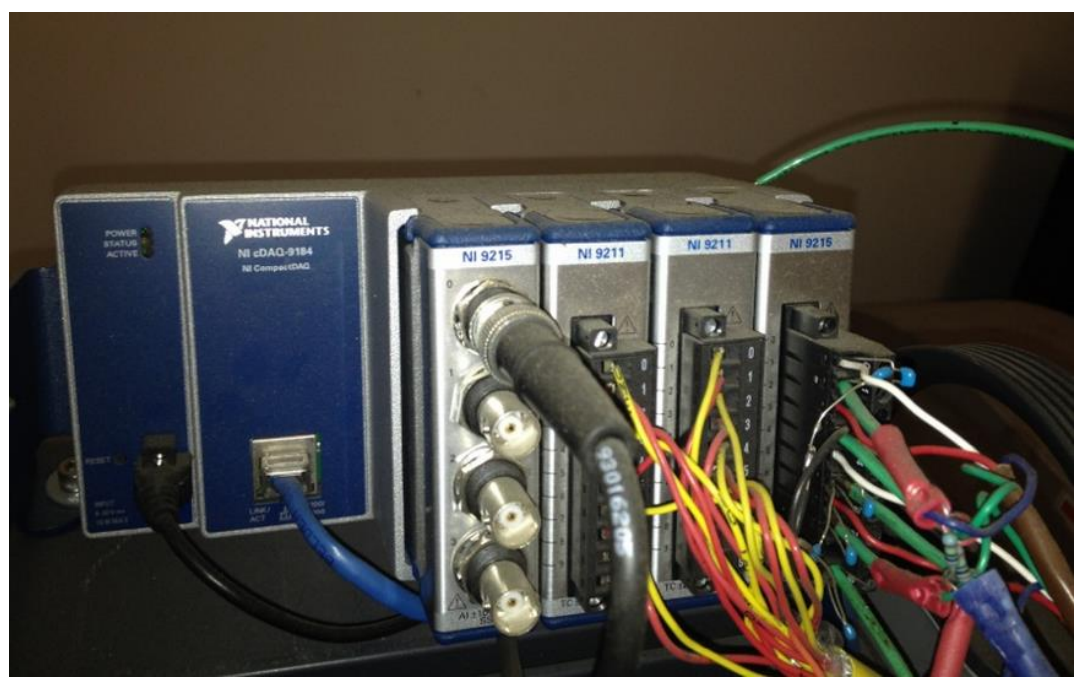

Figure 31: National Instruments DAQ with Cards

\subsubsection{HOTWIRE ANEMOMETER AND PITOT-STATIC PROBE}

Turbulence data was acquired via a Dantec Dynamics $5 \mu \mathrm{m}$ one-dimensional gold plated hotwire anemometer (Figure 32). The hotwire anemometer functions by adjusting the supplied current to maintain a constant wire temperature as air passes over the probe. The probe is connected as part of a Wheatstone bridge, and the bridge voltage is a representation of the heat transfer over the wire resulting in a direct measurement of velocity through a convection correction. The probe, rated for a maximum sampling rate of $400 \mathrm{kHz}$, and Wheatstone bridge are connected to a National Instruments 9215 BNC 4 channel analog input module with a maximum sampling rate of $100 \mathrm{kHz}$. To satisfy the Nyquist Sampling Frequency Criterion data was collected at $50 \mathrm{kHz}$. The Nyquist criterion states that when the sampling rate is less than twice the highest possible frequency, the reconstruction can represent a false image of the true signal resulting in false measurements known as aliasing. The sampling rate was found based off of the theoretical eddy integral length scale based on the turbulence grid bar diameters and the mean flow velocity. The process to find the true measured length scale will be presented in 3.1. 
The hotwire anemometer was calibrated with the pitot-static probe through a large velocity range and applied to a King's Law curve fit as can be seen in equation (2-4). This velocity range was largely dependent upon the anticipated freestream turbulence intensity as well as the freestream flow velocity.

$$
\mathrm{U}^{\mathrm{n}}=m V^{2}+\mathrm{b}
$$

Here $\mathrm{V}$ is the hotwire voltage, $\mathrm{m}$ is the slope of the calibration curve, $\mathrm{b}$ is a given calibration constant, and $\mathrm{n}$ is the King's Law exponent typically between (0.45 and 0.5$)$. The freestream velocity $\mathrm{U}$ measured by the pitot-static probe is found through the Bernoulli Equation (2-5):

$$
\mathrm{P}_{\text {static }}+\left(\rho \frac{\mathrm{U}^{2}}{2}\right)=\mathrm{P}_{\text {total }}
$$

And with manipulation leads to equation (2-6):

$$
\mathrm{U}=\sqrt{\frac{2 \Delta \mathrm{P}}{\rho}}, \text { where } \Delta \mathrm{P}=\mathrm{P}_{\text {total }}-\mathrm{P}_{\text {static }}
$$

Here $\mathrm{P}_{\text {static }}$ is the static pressure measured by the parallel flow around the pitot-static probe and $\left(\rho \mathrm{U}^{2} / 2\right)$ is the dynamic pressure measured at the leading edge of the probe. Turbulence characterization with the use of the hotwire anemometer will be covered in section 3.1.

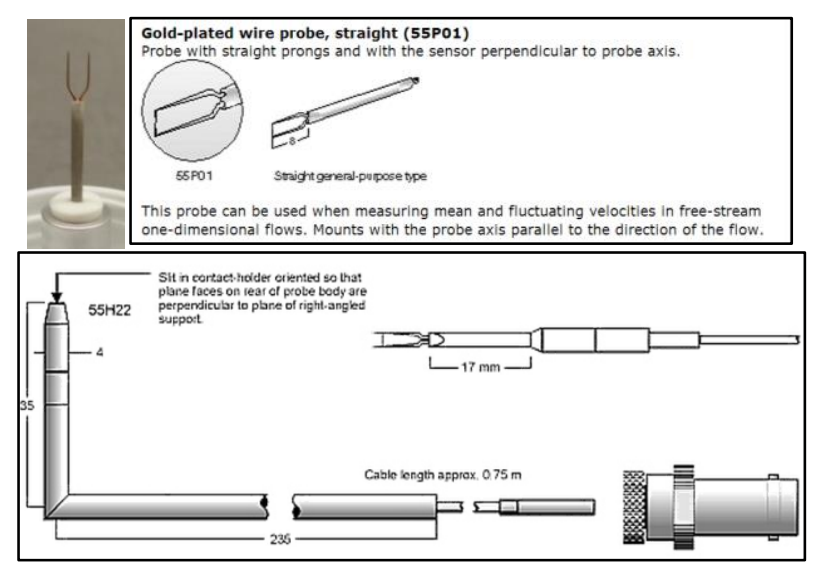

Figure 32: Dantec Dynamics Hotwire Anemometer Probe [43] 


\subsubsection{THERMOCOUPLES AND PRESSURE TRANSDUCERS}

\subsubsection{OMEGA THERMOCOUPLES}

The thermocouples used in this experiment were Omega Engineering KMQSS-020E-6 thermocouples. These were used to measure the freestream, surface, LFE, and plenum temperatures respectively. This K-type thermocouple is comprised of CHROMEGA ${ }^{\circledR}$ and ALOMEGA $^{\circledR}$ which are a chromium-nickel alloy (positive leg) and an aluminum-nickel alloy (negative leg). These thermocouples are one of the most common types of thermocouples, and are an adequate choice for many applications. Those used in the facility had exposed tips which allowed for a minute connection $(<0.020$ ") to be made between both metals allowing for a faster response (time constant $<0.5$ seconds) to temperature change relative to a larger diameter junction or a covered tip, so that the response time of the thermocouples would not lag behind that of the infrared camera. These have special error limits of the greater of either $\pm 1.1^{\circ} \mathrm{C}$ or $0.4 \%$.

Eight of these K-type thermocouples were placed in the facility. One was placed just upstream of the LFE to measure the incoming temperature, one was located in the freestream flow, five were installed on the test surface, with one specifically located in the lowest temperature area and one located in the hottest area to properly calibrate the IR camera, and the final thermocouple was located in the plenum for coolant flow temperature measurements.

\subsubsection{OMEGA PRESSURE TRANSDUCERS}

An Omega PX277-30D5V differential pressure transducer was used to measure the differential pressure across the laminar flow element as discussed in the coolant loop section. This transducer was capable of six different field selectable ranges allowing for 
flexibility if changes to the facility had to be made. An Omega PX277-05D5V differential transducer was also used to measure the differential pressure produced by the pitot-static probe for velocity measurements as well as the dynamic pressure for the boundary layer probe where the low pressure side was left at atmospheric pressure. The accuracy of these two transducers was $\pm 1 \%$ of the selected field. The LFE upstream pressure was measured by an Omega PX309-050A5V absolute pressure transducer while the plenum pressure was measured by an Omega PX309-030A5V absolute pressure transducer. The accuracy of these two absolute pressure transducers was $\pm 0.25 \%$ of the "frequency response best straight line". All pressure transducers produced an output voltage linearly related to the pressure measured and was connected to an NI 9215 four channel analog input card. Power was supplied to all transducers through a 0-30V at 0-3A variable bench-top power supply.

\subsubsection{INFRARED CAMERA}

To start with a rather broad and generalized fact, all matter above absolute zero temperature emits radiation. The amount of radiation emitted from an object varies with its temperature. In an ideal situation the object of interest would be one that absorbs all of the radiation that impinges upon it and therefore would emit the greatest amount of energy; this ideal object is commonly referred to as a blackbody. In real applications there is no such thing as a true blackbody, although some objects do come close for certain wavelength bands and ranges. There are three factors that prevent an object from acting like a blackbody: the ratio of incident radiation that is absorbed by the object $(\alpha)$, the ratio of incident radiation that is reflected by the object $(\rho)$, and the ratio of incident radiation that is transmitted into the object $(\tau)$. The total radiation law shows the relation of the three factors 
incorporating the subscript $(\lambda)$ to denote that these values are wavelength dependent and is shown as:

$$
1=\alpha_{\lambda}+\rho_{\lambda}+\tau_{\lambda}
$$

Here each value can vary from 0 to 1 and in the case of a blackbody resulting in $\alpha=1, \rho=$ 0 , and $\tau=0$. When the object is opaque, the radiation transmitted term falls out resulting in:

$$
1=\alpha_{\lambda}+\rho_{\lambda}
$$

One more parameter must be introduced to describe the ratio of spectral radiant power from a source compared to that of a blackbody for a given wavelength and temperature. This is known more specifically as emissivity $(\varepsilon)$ and is expressed mathematically as:

$$
\varepsilon_{\lambda}=\frac{\mathrm{W}_{\lambda, \text { object }}}{\mathrm{W}_{\lambda, \text { blackbody }}}
$$

For blackbodies, the above emissivity is equal to one while the graybodies have an emissivity of less than one.

Kirchhoff's law states that at a specified temperature and wavelength the objects emissivity and spectral absorbance are equal which is to say:

$$
\varepsilon_{\lambda}=\alpha_{\lambda}
$$

So for opaque materials the equation can be shown as:

$$
1=\varepsilon_{\lambda}+\rho_{\lambda}
$$

And highly polished materials that are nearly perfect reflectors are shown as:

$$
1=\rho_{\lambda}
$$

The wall temperature data collection for the heat transfer and film cooling effectiveness was obtained through the use of a FLIR A655sc (Figure 33) infrared camera which uses an uncooled mircobolometer with a spectral range of 7.5 to $14 \mu \mathrm{m}$ residing in the Long Wave Infrared (LWIR) band. The camera has a resolution of $640 \times 480$ pixels with each pixel 
being 17 microns in size, and is capable of recording 25 frames per second with a USB connection. Camera precision was $\pm 2^{\circ} \mathrm{C}$ or $\pm 2 \%$ of the reading (whichever is larger) of the camera, and this precision was further increased via calibration with the five surface thermocouples described in the previous section. LWIR is ideal for the measurements because LWIR is most appropriate for measurements around "room temperature" and is also ideal for this facility because it is far less susceptible to scatter due to fog, smoke, and dust making it an ideal candidate for future contemporaneous use with systems such as Laser Doppler Velocimetry (LDV) or Particle Image Velocimetry (PIV) systems.

The temperature of the test section surface was viewed through a polyethylene window which had an approximate thickness of $\leq 12.5 \mu \mathrm{m}$ and a calculated transmissivity of 0.74 . The camera set up is shown in Figure 34. The emissivity of the flat black painted test surface was found to be 0.95 . Additionally, a near blackbody light shroud surrounds the camera, as to reduce the atmospheric radiation reaching the volume of the test section and interfering with measurements.
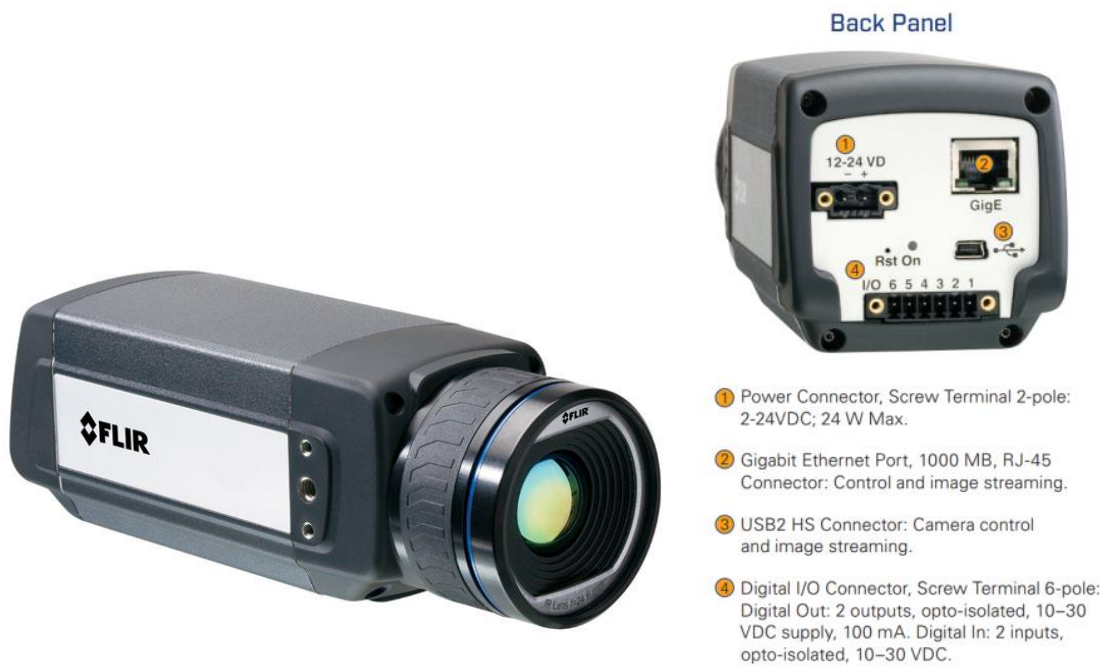

Figure 33: FLIR A655sc Infrared Camera [44] 
The IR camera was controlled and data was recorded through FLIR ExaminIR Pro ${ }^{\circledR}$ software. The user is responsible for taking into account and entering into the program the object emissivity, the distance from the surface of interest to the lens of the camera, the ambient temperature of the surrounding objects that reflect on the object of interest $\left(\mathrm{T}_{\text {reflect }}\right)$, the relative humidity of the atmosphere, the transmissivity of the atmosphere $\left(\tau_{\mathrm{atm}}\right)$, the temperature of the atmosphere $\left(\mathrm{T}_{\mathrm{atm}}\right)$, the window temperature $\left(\mathrm{T}_{\text {window }}\right)$, and window transmissivity $\left(\tau_{\text {window }}\right)$.

The camera is calibrated with the five surface thermocouples by matching the given temperature of each thermocouple with that of the camera by changing the window temperature due to the non-uniformity that exists in a transient test. The transient test is discussed and outlined in Chapter 3. The leading edge of the window experiences a faster temperature change than the downstream area of the window. Once the five temperatures are found, the average window temperature is used and applied to the current camera frame to be selected for use in the methodology presented in sections 3.2 and 3.3. Furthermore, the camera timestamp and the computer/DAQ timestamp are both recorded so that the initiation of the transience can be matched between both systems as a trigger could not be connected between both recording devices which is important again for the methodology presented in sections 3.2 and 3.3. 

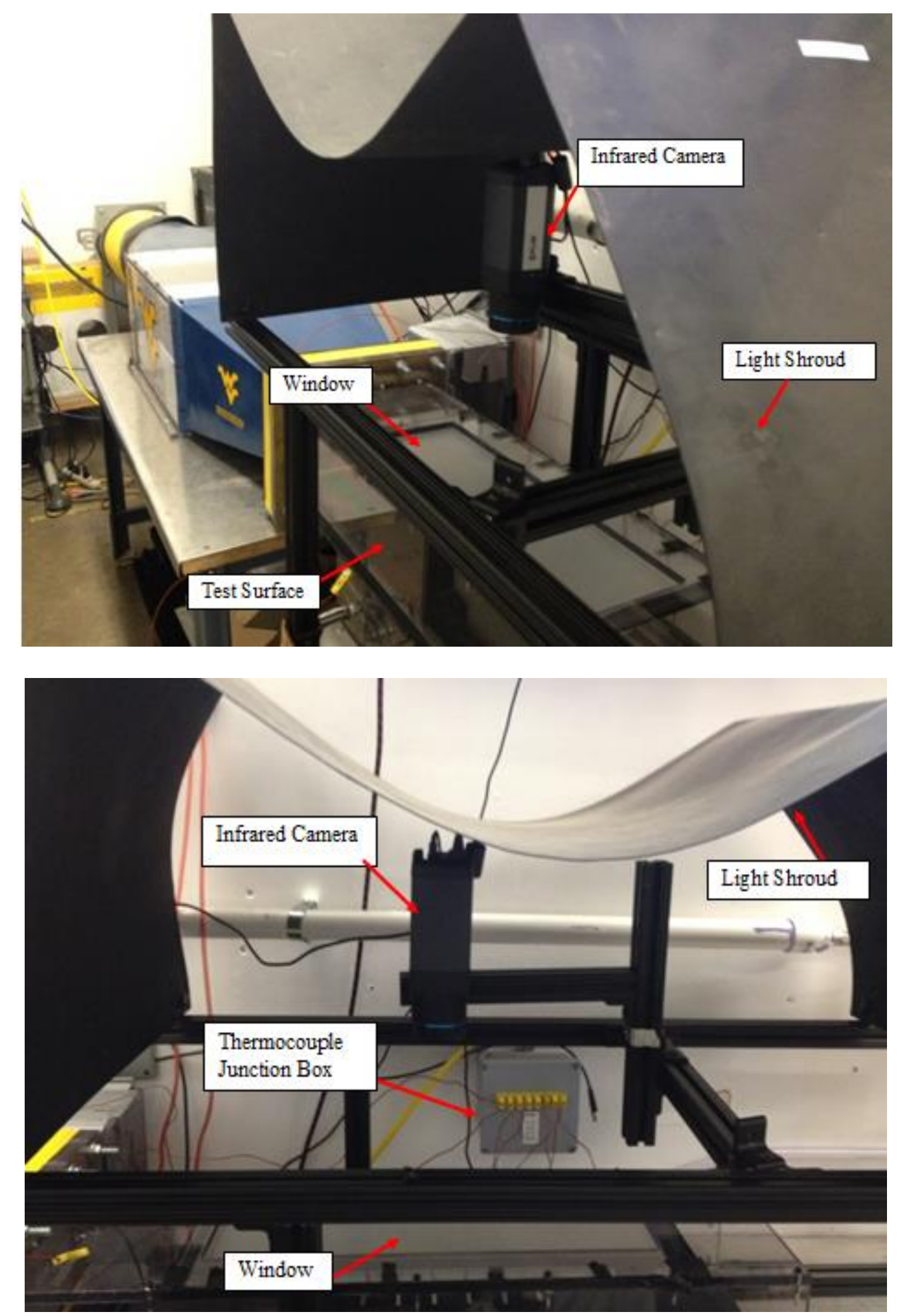

Figure 34: Camera Mounted during Transient Tests 


\section{CHAPTER 3: EXPERIMENTAL METHODOLOGY}

\subsection{TURBULENCE CHARACTERIZATION}

As outlined in section 2.2.1 hotwire data were collected at $50 \mathrm{kHz}$ for ten seconds to find the flow velocity, freestream turbulence intensity, and turbulence length scale. The fact that turbulence is random and chaotic requires that the mean and fluctuating components must be measured to give the best idea of flow characteristics. Figure 35 illustrates an example of the time-resolved velocity data collected by the hotwire anemometer broken down into two components: the mean velocity $(\overline{\mathrm{U}})$ and the fluctuating component $\left(\mathrm{u}^{\prime}\right)$. The measured velocity (U) at a given time is a combination of the two components given in equation (3-1) as:

$$
\mathrm{U}=\overline{\mathrm{U}}+\mathrm{u}^{\prime}
$$
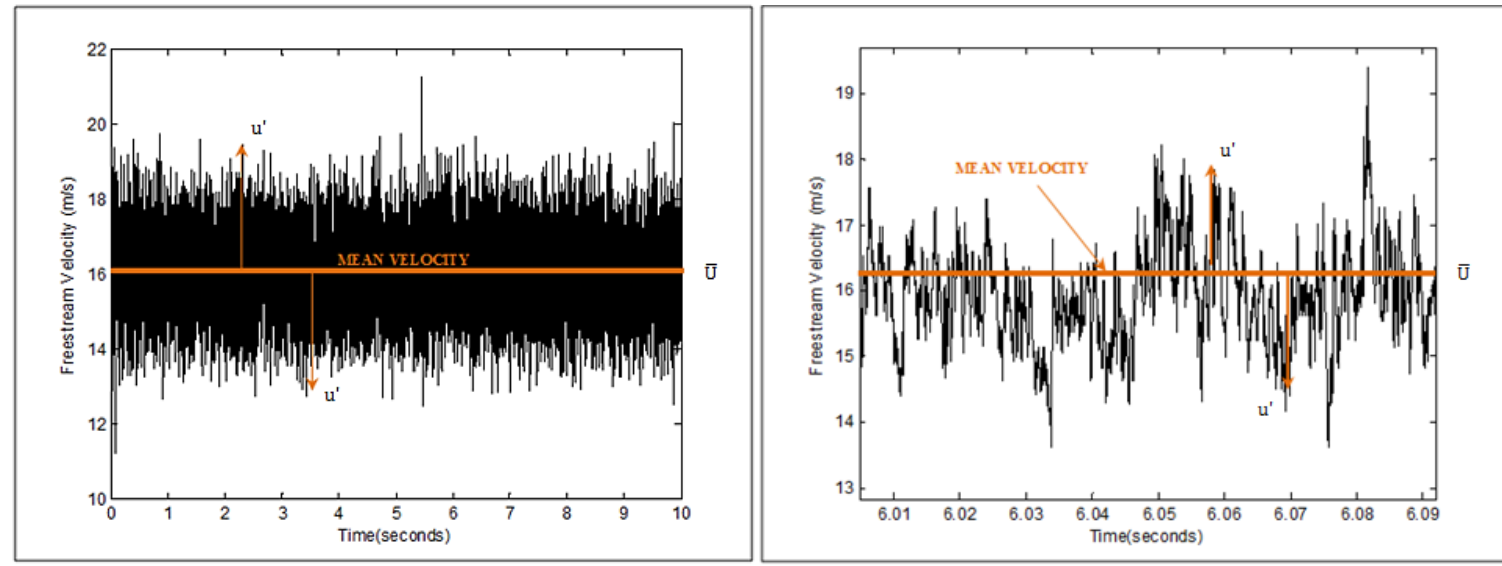

Figure 35: Hotwire Velocity Data

Figure 35 additionally shows the signal "fuzziness" which is a result of the multiple length scales of turbulence. Once zoomed in as in the right side case, the smaller length scales become evident much more so than when viewing the data over the entire time 
domain. The freestream turbulence intensity $(\mathrm{Tu})$ can be determined through the decomposition of the signal by using both components and can be shown as:

$$
\mathrm{Tu}=\frac{\sqrt{\overline{\mathrm{u}^{\prime 2}}}}{\overline{\mathrm{U}}}=\frac{\mathrm{u}_{\mathrm{RMS}}^{\prime}}{\overline{\mathrm{U}}}
$$

Here the value $u_{\text {RMS }}^{\prime}$ is the root mean square (RMS) of the fluctuation term at every time instant and can be determined in the time domain as:

$$
\mathrm{u}_{\mathrm{RMS}}^{\prime}=\sqrt{\frac{1}{\mathrm{~N}} \sum_{\mathrm{i}=1}^{\mathrm{N}} \mathrm{u}_{\mathrm{i}}^{\prime 2}}
$$

In the above equation $\mathrm{i}$ is the individual data point of the $\mathrm{N}$ data points.

The RMS value can also be determined in the frequency domain using the power spectral density (PSD) for the fluctuating velocity component. The PSD provides a representation of the dependence of energy on the frequency of fluctuation for an eddy scale. Using a fast Fourier transform (FFT) to decompose the fluctuating velocity component of the signal, the RMS value can be found and thus the turbulence intensity can be determined. An example PSD is shown in Figure 36 from the velocity signal presented in Figure 34. 


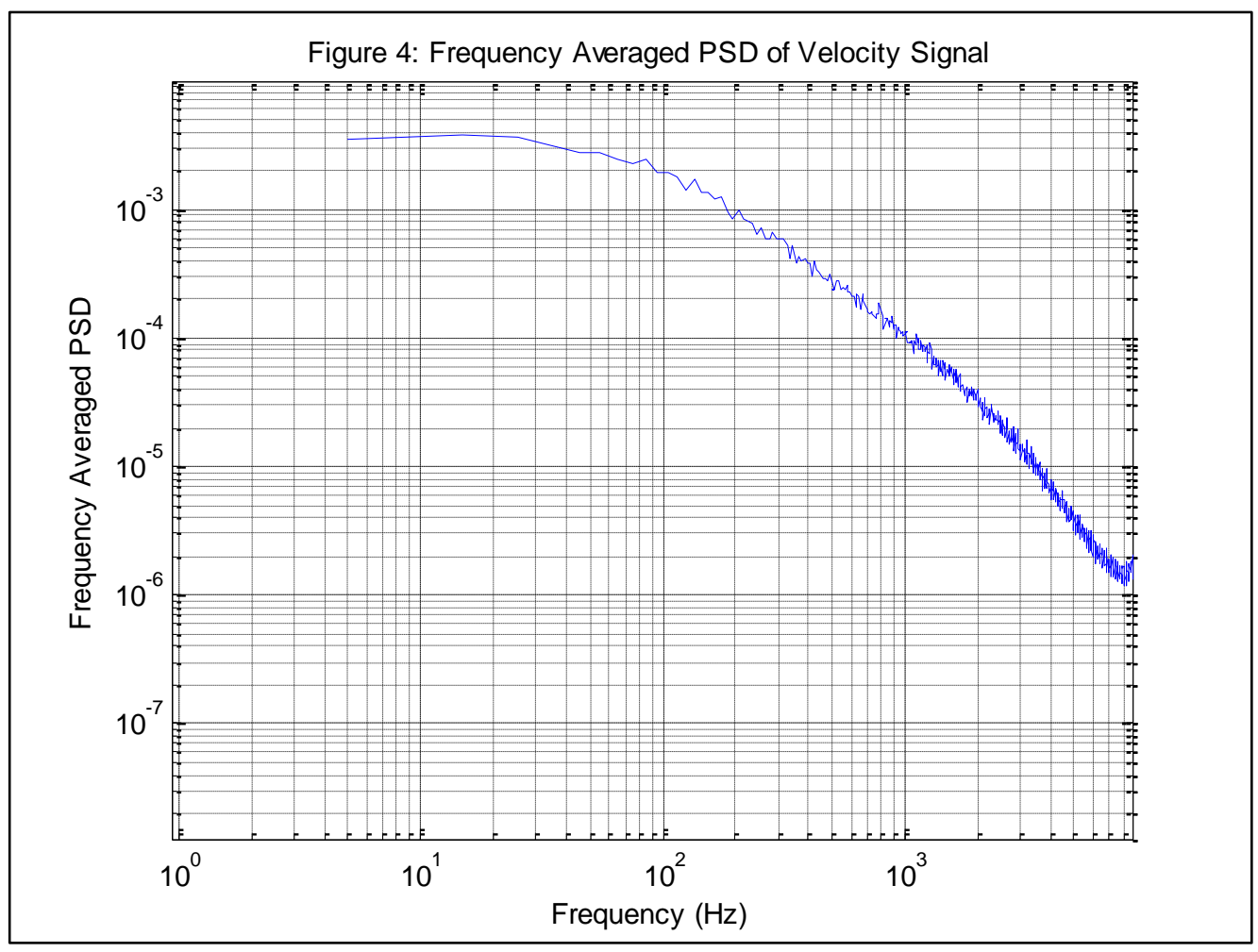

Figure 36: Velocity Power Spectral Density (PSD) from Hotwire Data

The length scale found is the integral length scale which are the largest turbulent eddies and contain the most energy as discussed in Appendix A along with other turbulence characteristics. The method used to determine the length scale of the turbulent eddies is the autocorrelation technique as presented by Nix [45]. The autocorrelation $\left(\mathrm{R}_{11}\right)$ of the fluctuating velocity component can be used to calculate the integral time scale $(T)$ by integrating under the curve to the first zero crossing. Equation (3-4) shows the autocorrelation equation where, as the lag time $(\tau)$ goes to zero, the RMS value of the velocity signal squared yields the unnormalized value of the autocorrelation.

$$
\mathrm{R}_{11}(\tau)=\left(\overline{\frac{\mathrm{u}^{\prime}(\mathrm{t}) * \mathrm{u} \prime(\mathrm{t}+\tau)}{\mathrm{u}^{\prime} \mathrm{RMS}^{2}}}\right)=\frac{1}{\mathrm{~N}}\left(\frac{\sum_{\mathrm{i}=1}^{\mathrm{N}} \mathrm{u}^{\prime} * \mathrm{u}^{\prime} \mathrm{i}+\mathrm{j}}{\mathrm{u}^{\prime}{ }_{\mathrm{RMS}}{ }^{2}}\right) \text {, where } \tau=\mathrm{j}^{*} \Delta \mathrm{t}
$$

Here $\mathrm{j}$ is the number of time steps and $\Delta \mathrm{t}$ is the size of the time steps. 

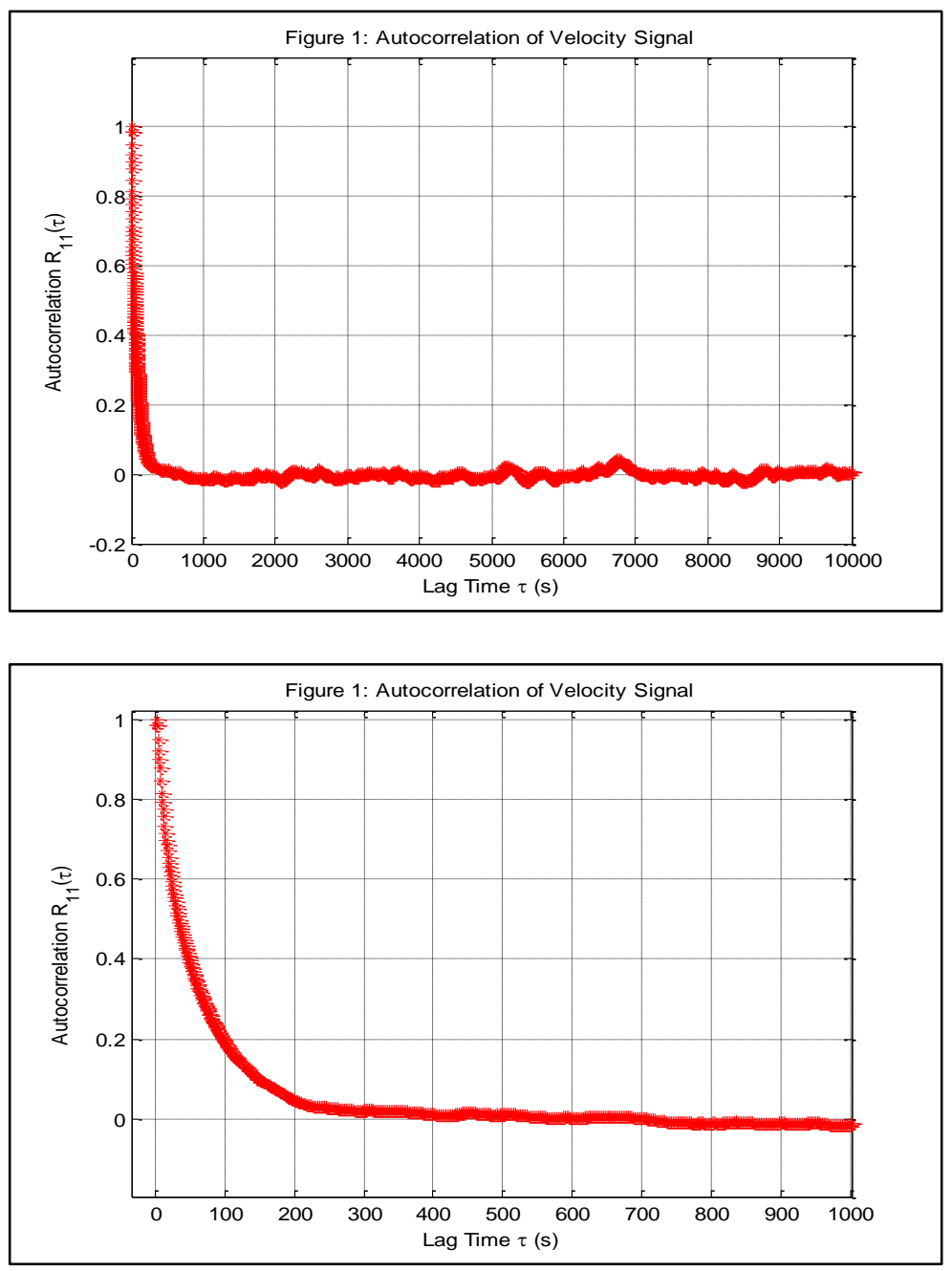

Figure 37: Autocorrelation Curve and Zoomed in Region of Interest (First Crossing)

Figure 37 shows an autocorrelation curve for the fluctuating velocity signal with a zoomed in section showing the first crossing region, again for the velocity data shown in Figure 35 . The time scale can be found with equation (3-5) where $\mathrm{N}_{0}$ is the location of the first zero crossing in the autocorrelation.

$$
T=\int_{\mathrm{t}=0}^{\infty} \mathrm{R}_{11}(\tau) \mathrm{d} \tau=\sum_{\mathrm{i}=1}^{\mathrm{N}_{0}} \mathrm{R}_{11_{\mathrm{i}}} * \Delta \tau
$$

If there were errors in the measurement due to vibrations of the hotwire or electrical noise the autocorrelation value would have higher peaks and valleys after reaching the zero 
crossing and could continue to the end of the plot. With the time scale now accounted for, the streamwise integral length scale can be found by multiplying the time scale by the mean velocity show in equation (3-6).

$$
\Lambda=\overline{\mathrm{U}} * T
$$

This equation is assumed to be valid as described by Taylor [46] for a measurement at a single point in space, for a flow with a non-zero convective mean velocity and is referred to as "frozen turbulence".

The above was used to determine the freestream turbulence intensity ( $\mathrm{Tu}$ ) and freestream turbulent length scale $(\Lambda)$ of the three turbulence conditions of no turbulence grid, small bar diameter grid, and large bar diameter grid as described in section 2.1.1.1.

\subsection{TRANSIENT HEAT TRANSFER}

\subsubsection{TRANSIENT FLOW OVER A FLAT PLATE}

The transient case of a flow passing over a semi-infinite solid flat plate is presented in Figure 38, and is known as a two temperature problem where the freestream temperature and wall temperature are the only drivers for heat transfer. In principal the surface can be thought of as infinite in all but one direction $(\mathrm{z})$, which is considered to be semi-infinite. This allows one to assume that when a sudden change occurs at the surface a transient onedimensional conduction into the solid occurs as a result. 


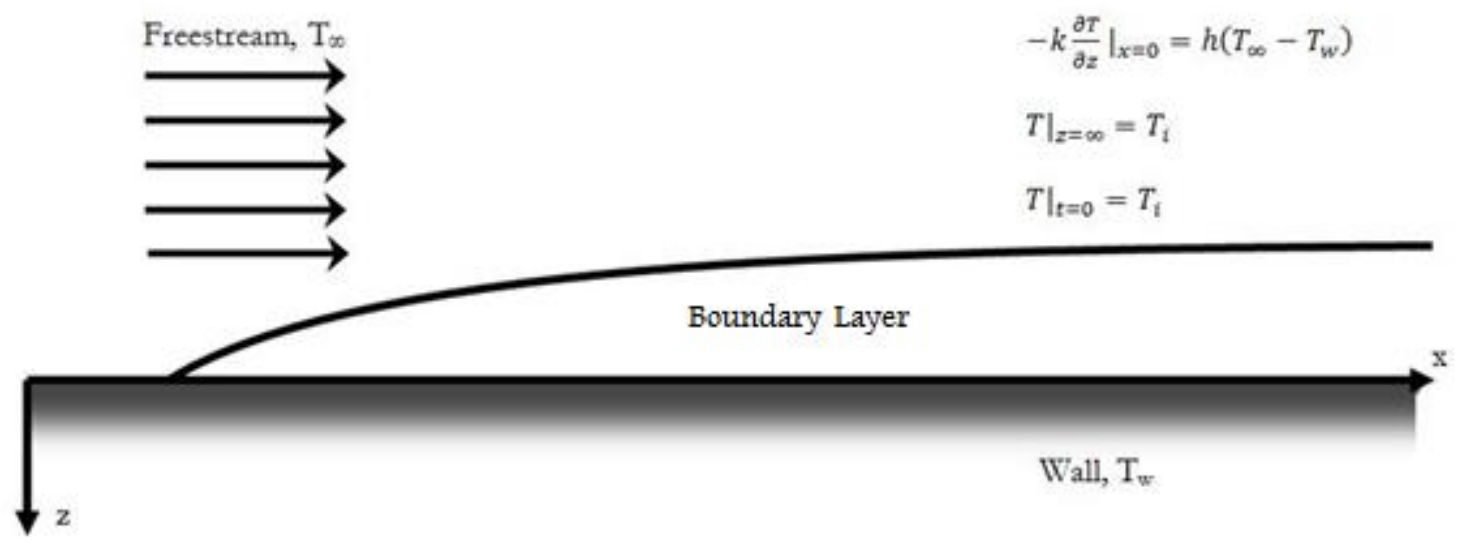

Figure 38: Transient Convection Heat Transfer over a Semi-Infinite Solid Flat Plate

The governing equation for one-dimensional unsteady heat conduction is:

$$
\mathrm{k} \frac{\partial^{2} \mathrm{y}}{\partial \mathrm{z}^{2}}=\rho \mathrm{C}_{\mathrm{p}} \frac{\partial \mathrm{T}}{\partial \mathrm{t}} \quad \text { or } \quad \frac{\partial^{2} \mathrm{y}}{\partial \mathrm{z}^{2}}=\frac{1}{\alpha} \frac{\partial \mathrm{T}}{\partial \mathrm{t}}, \quad \text { where } \alpha=\frac{\mathrm{k}}{\rho \mathrm{C}_{\mathrm{p}}}
$$

The boundary and initial conditions for this case are:

$$
\begin{gathered}
-\mathrm{k} \frac{\partial \mathrm{T}}{\partial \mathrm{z}}=\mathrm{h}\left(\mathrm{T}_{\infty}-\mathrm{T}(0, \mathrm{t})\right) \\
\left.\mathrm{T}\right|_{\mathrm{z}=\infty}=\mathrm{T}_{\mathrm{i}} \\
\left.\mathrm{T}\right|_{\mathrm{t}=0}=\mathrm{T}_{\mathrm{i}}
\end{gathered}
$$

Here $T_{i}$ is the initial uniform wall temperature, $T_{\infty}$ is the freestream flow, and $T_{w}$ the varying wall temperature through the transient conduction.

The assumption that the plate is a semi-infinite solid means that the heat transfer into the top of the plate cannot be experienced by the bottom of the plate during the experiment. This can be measured by the penetration depth which is given in equation (3-11):

$$
\text { Penetration Depth }=\sqrt{\alpha \mathrm{t}}
$$

Here $\left(\alpha=0.1076^{*} 10^{-6} \mathrm{~m}^{2} / \mathrm{s}\right)$ is the thermal diffusivity of the ABS plastic and (t) is the time of the transience which was 60 seconds. Solving this equation yields a penetration depth of 
approximately 0.1 inches. In the current experiment, the tunnel bottom wall and test plate are 1 inch thick so this assumption is adequately satisfied.

Solving equation (3-7) with the outlined conditions yields:

$$
\frac{\mathrm{T}_{\mathrm{w}}-\mathrm{T}_{\mathrm{i}}}{\mathrm{T}_{\infty}-\mathrm{T}_{\mathrm{i}}}=1-\exp \left(\frac{\mathrm{h}^{2} \alpha \mathrm{t}}{\mathrm{k}^{2}}\right) \operatorname{erfc}\left(\frac{\mathrm{h} \sqrt{\alpha \mathrm{t}}}{\mathrm{k}}\right)=1-\operatorname{erfcx}\left(\frac{\mathrm{h} \sqrt{\alpha \mathrm{t}}}{\mathrm{k}}\right)
$$

Here (h) is the unknown heat transfer coefficient, again $(\alpha)$ is the thermal diffusivity of the ABS plastic, (k) is the thermal conductivity of the ABS plastic, and (t) is the duration of time from the initiation of the transient test. Equation (3-12) above is furthermore applicable because a true step temperature change is imposed upon the freestream flow.

\subsubsection{TRANSIENT FILM COOLING}

As stated previously, equation (3-12) is for a two temperature problem, but film cooling is actually a three temperature problem where the freestream temperature, wall temperature, and injected coolant temperature drive the heat transfer and can be seen in Figure 39. Equation (3-12) must be adjusted to incorporate the film temperature from the coolant and freestream interacting and results in:

$$
\frac{\mathrm{T}_{\mathrm{w}}-\mathrm{T}_{\mathrm{i}}}{\mathrm{T}_{\mathrm{f}}-\mathrm{T}_{\mathrm{i}}}=1-\operatorname{erfcx}\left(\frac{\mathrm{h} \sqrt{\alpha \mathrm{t}}}{\mathrm{k}}\right)
$$

where the complementary error function is:

$$
\operatorname{erfc}(x)=1-\operatorname{erf}(x)
$$

and the scaled complementary error function is:

$$
\operatorname{erfcx}(x)=\exp \left(x^{2}\right) * \operatorname{erf}(x)
$$




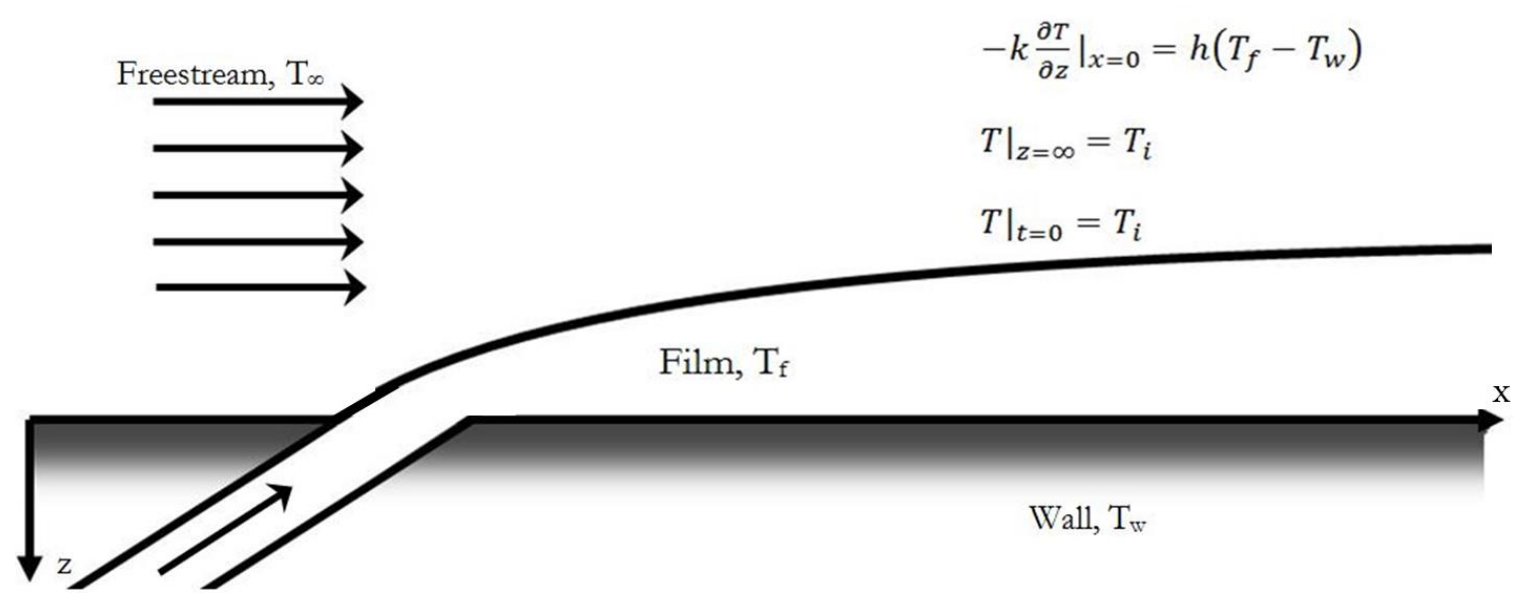

Figure 39: Transient Film Cooling over a Semi-Infinite Solid Flat Plate

Equation (3-13) has two unknown values which are the film temperature $\left(T_{f}\right)$ and convective heat transfer coefficient (h), but of greater interest than the film temperature is another variable which is based on the film temperature and is known as film cooling effectiveness $(\eta)$. The highest theoretically possible value of film cooling effectiveness is one (1) when the film temperature is equal to the coolant temperature and the lowest value of zero (0) occurs when the film temperature is equal to the freestream temperature. Film cooling effectiveness is a non-dimensional term and can be shown as:

$$
\eta=\frac{T_{f}-T_{\infty}}{T_{c}-T_{\infty}} \text { or } \eta_{a w}=\frac{T_{\infty}-T_{a w}}{T_{\infty}-T_{c}} \text { eq:(1-1) }
$$

Rearranging to solve for $\mathrm{T}_{\mathrm{f}}$ :

$$
\mathrm{T}_{\mathrm{f}}=\eta *\left(\mathrm{~T}_{\mathrm{c}}-\mathrm{T}_{\infty}\right)+\mathrm{T}_{\infty}=\eta * \mathrm{~T}_{\mathrm{c}}+(1-\eta) * \mathrm{~T}_{\infty}
$$

Taking equation (3-13) and solving for $T_{w}-T_{i}$ and replacing $T_{f}$ with equation (3-17) yields:

$$
\mathrm{T}_{\mathrm{w}}-\mathrm{T}_{\mathrm{i}}=1-\operatorname{erfc}\left(\frac{\mathrm{h} \sqrt{\alpha \mathrm{t}}}{\mathrm{k}}\right) *\left[\eta * \mathrm{~T}_{\mathrm{c}}+(1-\eta) * \mathrm{~T}_{\infty}-\mathrm{T}_{\mathrm{i}}\right]
$$

with the two desired unknowns being the heat transfer coefficient and the film cooling effectiveness. 
Ekkad et al. [47] proposed utilizing infrared thermography in a method previously used by Vendula and Metzger [48]. Vendula and Metzger used thermochromatic liquid crystal paint wherein two different time instances from a single transient test could be used to simultaneously solve a system of equations with two unknowns ( $h$ and $T_{f}$ ). These two equations occur at the time instant one $\left(\mathrm{t}_{1}\right)$ with a wall temperature time $\left(\mathrm{T}_{\mathrm{w} 1}\right)$, and time instant two $\left(\mathrm{t}_{2}\right)$ with wall temperature $\left(\mathrm{T}_{\mathrm{w} 2}\right)$ and can be shown as:

$$
\begin{aligned}
& \frac{\mathrm{T}_{\mathrm{w} 1}-\mathrm{T}_{\mathrm{i}}}{\mathrm{T}_{\mathrm{f}}-\mathrm{T}_{\mathrm{i}}}=1-\operatorname{erfcx}\left(\frac{\mathrm{h} \sqrt{\alpha t_{1}}}{\mathrm{k}}\right) \\
& \frac{\mathrm{T}_{\mathrm{w} 2}-\mathrm{T}_{\mathrm{i}}}{\mathrm{T}_{\mathrm{f}}-\mathrm{T}_{\mathrm{i}}}=1-\operatorname{erfcx}\left(\frac{\mathrm{h} \sqrt{\alpha t_{2}}}{\mathrm{k}}\right)
\end{aligned}
$$

This method adequately solves for the correct values of the film temperature and heat transfer coefficient, and therefore the film cooling effectiveness from equation (3-16), but during early iterations of data reduction it was found that the resulting values were not

consistent if different frames were selected when separated. To resolve this issue a regression analysis was applied to compute reliable and consistent results based on the work done by $\mathrm{Lu}[49]$.

\subsection{REGRESSION ANALYSIS}

To reiterate, it is desired to solve for $\mathrm{h}$ and $\eta$, which can be done solving simultaneously the aforementioned two equations (3-19a and 3-19b) with these two unknowns, but the desire is to minimize the resulting uncertainty as much as possible.

The transient test is initiated when the coolant flow and step temperature change in the freestream are initiated simultaneously. For each test, surface temperature, freestream temperature, and coolant plenum temperature data are collected at $25 \mathrm{~Hz}$ and four frames are selected just before the transient surface temperature change begins for the initial wall 
temperature. Ten frames are selected six seconds apart through the 60 seconds of the transient test. To extract the data from the FLIR ExaminIR Pro ${ }^{\circledR}$ software, numerous data reduction steps must be performed to reduce error. Five surface thermocouples with known locations on the test surface are matched with their respective pixel on the FLIR recorded image. The locations of the thermocouples are shown below in Figure 40. Thermocouple 1 is located in the highest temperature region located upstream of the coolant holes and thermocouple 2 is located just downstream of the coolant hole in the coolest temperature region. This gives a temperature range in which the IR camera should be calibrated to allow for interpolation of the surface temperature values rather than an extrapolation which could result in higher errors in the lowest and highest temperature regions. Additionally, three thermocouples located further downstream were used to reduce errors associated with the temperature change that the window also undergoes.

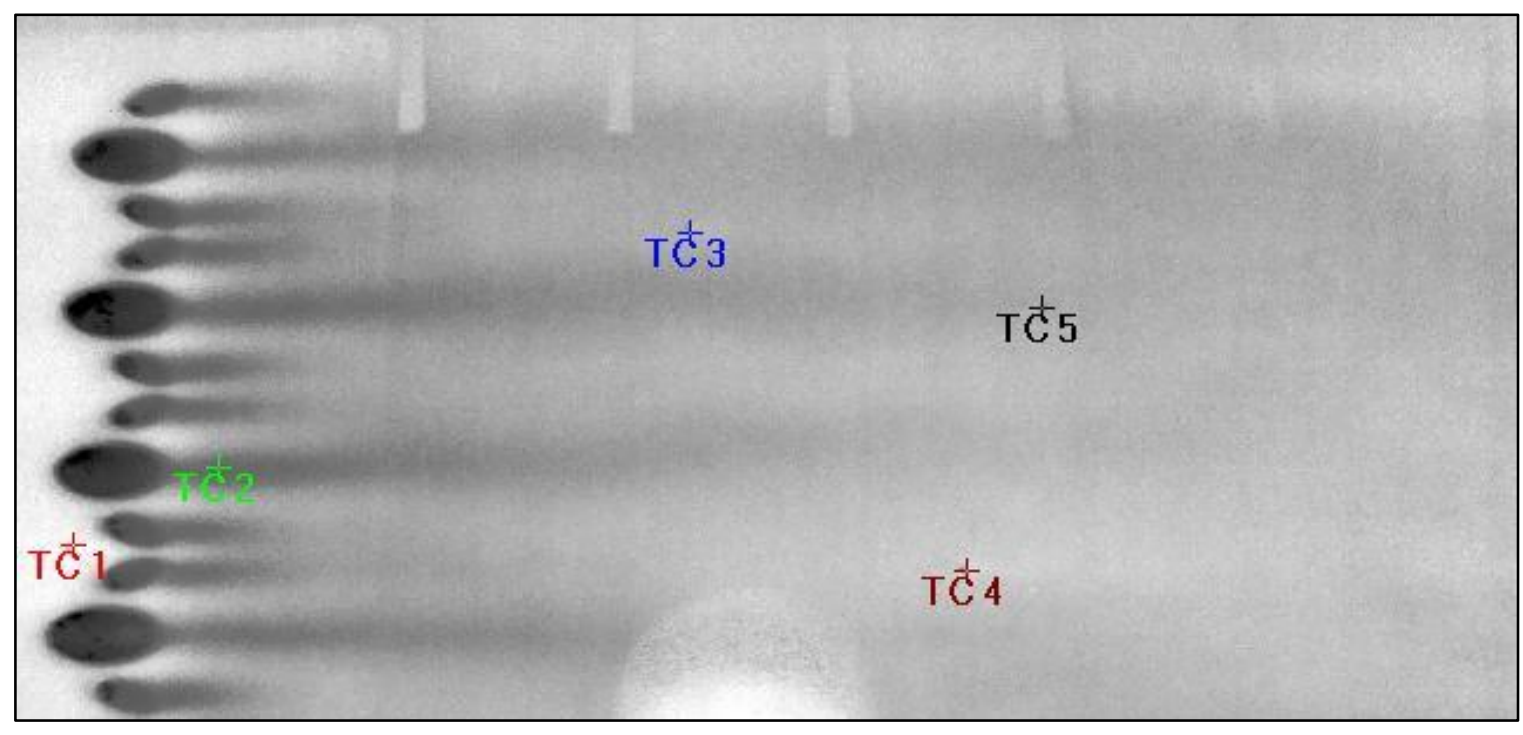

Figure 40: FLIR IR Image with Thermocouple Locations

Due to the step temperature change of the freestream, the polyethylene window undergoes a temperature change as well, resulting in a non-uniformity. For each thermocouple at one time instant, the external optics temperature is changed to match each 
thermocouple individually, then the average of the five temperatures is used for the external optics temperature (window temperature) and that frame is then output for further data reduction. The window temperature variation during transience is typically approximately $3^{\circ} \mathrm{C}$ for a particular frame and increases by approximately $0.5^{\circ} \mathrm{C}$ for each subsequent frame. After being applied to the four initial surface temperature frames before transient surface temperature change, and the ten during the transient surface temperature change, the thermocouple temperatures and radiance values are exported into a MATLAB ${ }^{\circledR}$ program where the MATLAB ${ }^{\circledR}$ "polyfit" function is used to perform a least squares regression resulting in new corrected temperatures which are applied to all 307,200 pixels (a single frame is $640 \times 480$ pixels) in the image reducing any error from camera and thermocouple readings. The four frames for the initial surface temperature are averaged together to attempt to account for any pixels that may have fluctuated due to camera errors, as bad pixel readouts may occur.

In an attempt to further reduce uncertainty, an iterative non-linear least-square regression analysis technique was used to solve for $\mathrm{h}$ and $\eta$ for a single experiment as presented by Lu [49]. From the previous steps, the known time at each frame $t_{I R}$ when a specific camera measured temperature value $T_{I R}$ appears during transience can be shown as:

$$
\left.\mathrm{T}_{\mathrm{w}}(\mathrm{h}, \eta)\right|_{\mathrm{t}=\mathrm{t}_{\mathrm{IR}}}=\mathrm{T}_{\mathrm{IR}}
$$

Where this equation applies separately to each individual pixel of each frame which can be expressed mathematically as:

$$
\left.\mathrm{T}_{\mathrm{w}}(\mathrm{h}, \eta)\right|_{\mathrm{t}=\mathrm{t}_{\mathrm{IR}}}-\mathrm{T}_{\mathrm{IR}}=0 \quad \forall_{\mathrm{i}}=1 \text { to } \mathrm{N}
$$

Here $\mathrm{i}$ is the given pixel and $\mathrm{N}$ is the number of frames being used. 
When more than two frames are being used to solve for $\mathrm{h}$ and $\eta$ that solves these equations with the least amount of error, the following error function is used

$$
\sum_{i=1}^{N}\left[\left.\mathrm{~T}_{\mathrm{w}}(\mathrm{h}, \eta)\right|_{\mathrm{t}=\mathrm{t}_{I R_{i}}}-\mathrm{T}_{I R_{i}}\right]^{2}=\mathrm{e}(\mathrm{h}, \eta) \geq 0
$$

Taking equation (3-22) and substituting in equation (3-18) allows for an iterative method to solve for $h$ and $\eta$ by minimizing the right hand side (the error). Using the "fminsearch" function in MATLAB $^{\circledR}$ and controlling the maximum number of function evaluations $(150,000)$ and maximum number of iterations $(100,000)$ per pixel and selecting initial guesses and upper and lower bounds for $h$ and $\eta$, these values could be computed. The process would take between 20 and 120 minutes depending upon the number of evaluations and iterations needed to reach the prescribed convergence tolerance of $1 * 10^{-5}$. This method reduces the error that is present in the infrared measurements and relaxes the dependence upon the initial wall temperature by applying additional time instances.

\subsection{EXPERIMENTAL UNCERTAINTY}

The experimental uncertainty analysis was performed based on the method outlined by Moffat [50] by introducing a bias uncertainty for each of the measured values and a precision uncertainty. The bias uncertainties were determined based on manufacture specifications and were perturbed in the regression code sequentially. The resulting values

of $\mathrm{h}$ and $\eta$ were determined at each pixel for the applied error. The values which were perturbed were: $\left(\mathrm{T}_{\infty}\right) \pm 0.5^{\circ} \mathrm{C},\left(\mathrm{T}_{\mathrm{c}}\right) \pm 0.5^{\circ} \mathrm{C},\left(\mathrm{T}_{\mathrm{i}}\right) \pm 0.125^{\circ} \mathrm{C},\left(\mathrm{T}_{\mathrm{w}}\right) \pm 0.125^{\circ} \mathrm{C},(\mathrm{t}) \pm 0.125 \mathrm{sec}$ and (k) $\pm 0.01 \mathrm{~W} /(\mathrm{m} \mathrm{K})$. The $\mathrm{T}_{\mathrm{w}}$ and $\mathrm{T}_{\mathrm{i}}$ temperature biases were each lower than the $\mathrm{T}_{\infty}$ and $\mathrm{T}_{\mathrm{c}}$ biases because a curve fit was applied between the five surface thermocouples. Further, the assumption was made that not all thermocouples could have a $+0.5^{\circ} \mathrm{C}$ reading or $-0.5^{\circ} \mathrm{C}$ at 
the same time. An example will be outlined to demonstrate solving for the uncertainty in the film cooling effectiveness $(\eta)$. This method can be applied in a similar manner to the heat transfer coefficient (h).

Once all values had been perturbed and the codes had run, the positive perturbation $\left(\eta_{\mathrm{x}+}\right)$ and negative $\left(\eta_{\mathrm{x}-}\right)$ perturbation of each adjusted measurement were taken and averaged absolutely as can be seen in equations (3-22a-f).

$$
\begin{aligned}
\delta \eta_{\mathrm{T}_{\infty}} & =\frac{\left|\delta \eta_{\mathrm{T}_{\infty+}}\right|+\left|\delta \eta_{\mathrm{T}_{\infty}-}\right|}{2} \\
\delta \eta_{\mathrm{T}_{\mathrm{c}}} & =\frac{\left|\delta \eta_{\mathrm{T}_{\mathrm{c}+}}\right|+\left|\delta \eta_{\mathrm{T}_{\mathrm{c}-}}\right|}{2} \\
\delta \eta_{\mathrm{T}_{\mathrm{i}}} & =\frac{\left|\delta \eta_{\mathrm{T}_{\mathrm{i}+}}\right|+\left|\delta \eta_{\mathrm{T}_{\mathrm{i}-}}\right|}{2} \\
\delta \eta_{\mathrm{T}_{\mathrm{w}}} & =\frac{\left|\delta \eta_{\mathrm{T}_{\mathrm{w}+}}\right|+\left|\delta \eta_{\mathrm{T}_{\mathrm{w}-}}\right|}{2} \\
\delta \eta_{\mathrm{t}} & =\frac{\left|\delta \eta_{\mathrm{t}+}\right|+\left|\delta \eta_{\mathrm{t}-}\right|}{2} \\
\delta \eta_{\mathrm{k}} & =\frac{\left|\delta \eta_{\mathrm{k}+}\right|+\left|\delta \eta_{\mathrm{k}-}\right|}{2}
\end{aligned}
$$

These values are then used in finding the RMS value of the bias uncertainty shown in equation (3-23).

$$
\eta_{\text {bias }}=\sqrt{\left(\delta \eta_{\mathrm{T}_{\infty}}\right)^{2}+\left(\delta \eta_{\mathrm{T}_{\mathrm{c}}}\right)^{2}+\left(\delta \eta_{\mathrm{T}_{\mathrm{i}}}\right)^{2}+\left(\delta \eta_{\mathrm{T}_{\mathrm{w}}}\right)^{2}+\left(\delta \eta_{\mathrm{t}}\right)^{2}+\left(\delta \eta_{\mathrm{k}}\right)^{2}}
$$

The second part of uncertainty is the precision uncertainty shown as:

$$
\eta_{\text {precision }}=\frac{\Delta \eta}{\eta}=\frac{t * S_{\eta}}{\bar{\eta} * \sqrt{N-1}}
$$


Where $(\mathrm{t})$ is the Student- $\mathrm{t}$ variable $(\mathrm{t}=2.353)$ based on $\mathrm{N}-1$ tests, $\mathrm{N}$ is the number of tests $(\mathrm{N}=4)$ and $\mathrm{S}_{\eta}$ is the variance of the film cooling effectiveness.

Finally the uncertainty of the film cooling effectiveness can be determined from the bias and precision uncertainties shown in equation (3-25):

$$
\eta_{\text {uncertainty }}=\sqrt{\eta_{\text {bias }}^{2}+\eta_{\text {precision }}^{2}}
$$

The final uncertainties found were \pm 0.05 for centerline film cooling effectiveness and \pm 0.024 for span-averaged film cooling effectiveness. The convective heat transfer coefficient uncertainties were $\pm 28 \%$ for the centerline convective heat transfer coefficient and $\pm 33 \%$ for the span-averaged convective heat transfer coefficient. The bias uncertainty was found to be \pm 0.04 for centerline film cooling effectiveness and \pm 0.019 for spanaveraged film cooling effectiveness. The convective heat transfer coefficient bias uncertainties were $\pm 24 \%$ for the centerline convective heat transfer coefficient and $\pm 21 \%$ for the span-averaged convective heat transfer coefficient. The high uncertainty in the convective heat transfer coefficient could be due to the fact that the values had to be computationally solved rather than directly solved, but the comparative convective heat transfer values between tests are more important in determining trends between tests rather than those compared with other facilities. 


\section{CHAPTER 4: RESULTS AND DISCUSSION}

The following section will present benchmarking data of the conventional straight hole geometry including a test matrix to show experimental cases. The results will be presented in various formats including the centerline values, span-averaged values, area-averaged values, and as surface contour plots.

\subsection{BENCHMARKING RESULTS}

To ensure that the facility was functioning as anticipated, the $30^{\circ}$ inclined straight hole has been investigated, and the results for the film cooling effectiveness and convective heat transfer coefficient have been compared with those from previous studies with similar blowing ratios and freestream turbulence intensities. Table 2 depicts the benchmark testing performed to establish reliability and accuracy of the facility including data collection and processing.

Table 2: Straight Hole Benchmark Matrix

\begin{tabular}{|c|c|c|}
\hline Experiment & Blowing Ratio (BR) & Turbulence Intensity (Tu) \\
\hline $\mathbf{1}$ & 0.5 & $1 \%$ \\
\hline $\mathbf{2}$ & 0.5 & $7.5 \%$ \\
\hline $\mathbf{3}$ & 1.0 & $1 \%$ \\
\hline $\mathbf{4}$ & 1.0 & $7.5 \%$ \\
\hline $\mathbf{5}$ & 1.5 & $1 \%$ \\
\hline $\mathbf{6}$ & 1.5 & $7.5 \%$ \\
\hline $\mathbf{7}$ & 2.0 & $1 \%$ \\
\hline $\mathbf{8}$ & 2.0 & $7.5 \%$ \\
\hline
\end{tabular}




\subsubsection{FILM COOLING EFFECTIVENESS}

\subsubsection{SURFACE CONTOUR PLOTS}

Contour plots are used to present film cooling effectiveness results and assist in creating a better perspective of how the geometry is performing as a view of the entire surface can be offered. Figure 41 and Figure 42 show the contour plots of the film cooling effectiveness for the straight hole at blowing ratios of: $0.5,1.0,1.5$, and 2.0 and turbulence intensities of 1 and $7.5 \%$. Figure 41 displays the contour plots of constant freestream turbulence intensity while varying the blowing ratio while Figure 42 displays the contour plot of constant blowing ratio while varying the freestream turbulence intensity.
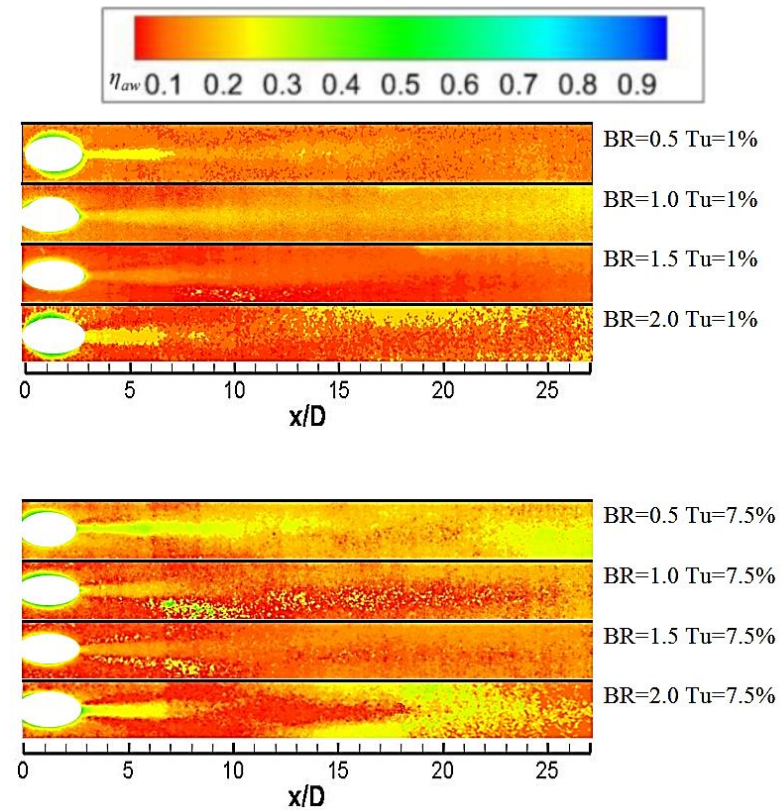

Figure 41: Film Cooling Effectiveness for Baseline Cases at Varying Blowing Ratio and Constant Turbulence Intensity 

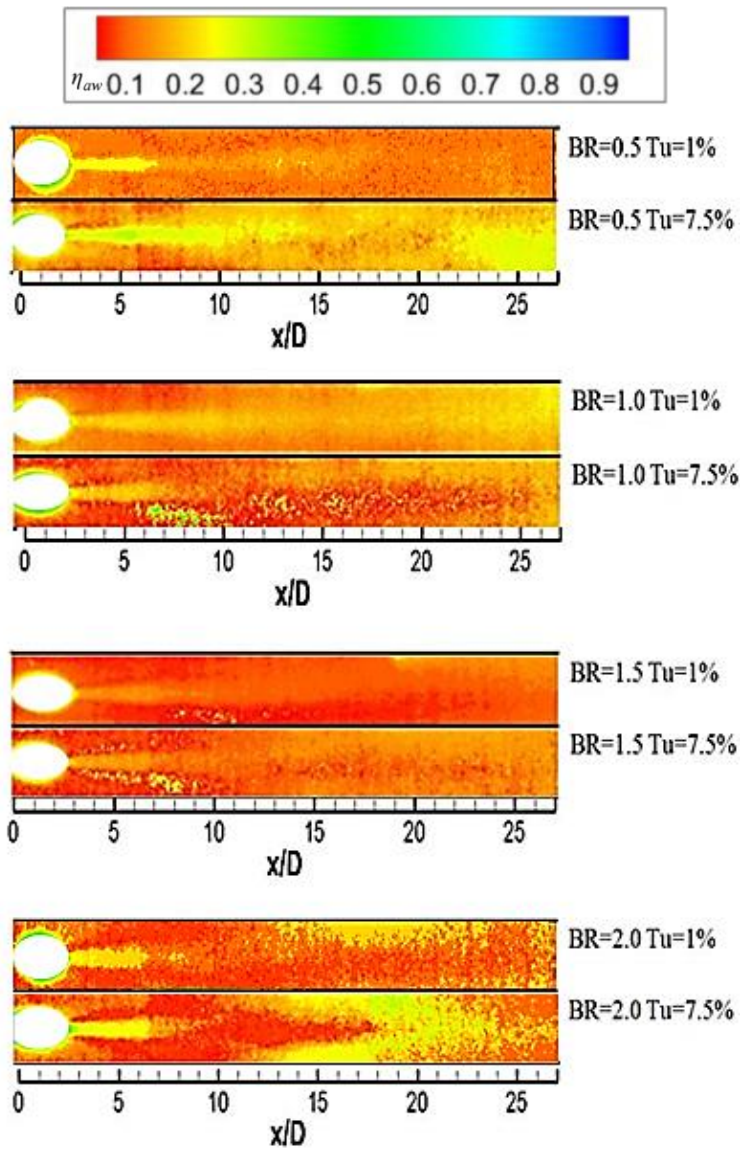

Figure 42: Film Cooling Effectiveness for Baseline Cases at Constant Blowing Ratio and Varying Turbulence Intensity

Generally speaking, the contour plots show indications of the coolant jet dispersing laterally for increased turbulence intensity although this is not directly apparent in all cases.

\subsubsection{SPAN-AVERAGED FILM COOLING EFFECTTIVENESS}

To present the data in a much clearer manner it is common practice to produce spanaveraged and centerline plots of film cooling effectiveness along the non-dimensionalized streamwise distance. The span-averaged results depict how well the film cooling geometry is performing. As previously mentioned, to benchmark the facility the current study must be compared to previously published literature. Three former studies were selected to compare with. 
Two of the previous studies $[13,35]$ were conducted using the transient infrared thermography technique similarly to the current study, and one was performed using the pressure sensitive paint (PSP) technique [29]. Dhungel et al. [13] conducted their study at a freestream Reynolds number based on cooling hole diameter of $\approx 9,700$ which is near the Reynolds number of the current study $(\approx 10,000)$. Their study also included $30^{\circ}$ inclined holes with hole pitch of three hole diameters and hole diameters of $1 / 2$ " with blowing ratios of $0.5,1.0,1.5$, and 2.0 which are all identical to the current study, but had a freestream turbulence intensity of $2 \%$ rather than the $1 \%$ freestream turbulence intensity in the current study. LeBlanc et al. [35] also performed their study with coolant holes inclined at $30^{\circ}$ with hole pitch of three hole diameters, but had coolant holes of $1 / 4$ " diameter resulting in a length over coolant hole diameter ratio (L/D) of 7.5 instead of 4 in the current study. Wright et al. [29] performed their study with $35^{\circ}$ inclined holes with a hole pitch of four hole diameters with a freestream turbulence intensity of $1.2 \%$ and a Reynolds number based on cooling hole diameter of $\approx 2,600$.

The span-averaged values for the current study compared with the previous studies can be seen in Figure 43. The current data resides between that of existing published literature for span-averaged film cooling. The effectiveness similarly displays the same trend of a decreasing effectiveness with an increasing blowing ratio as the previous studies indicated. 


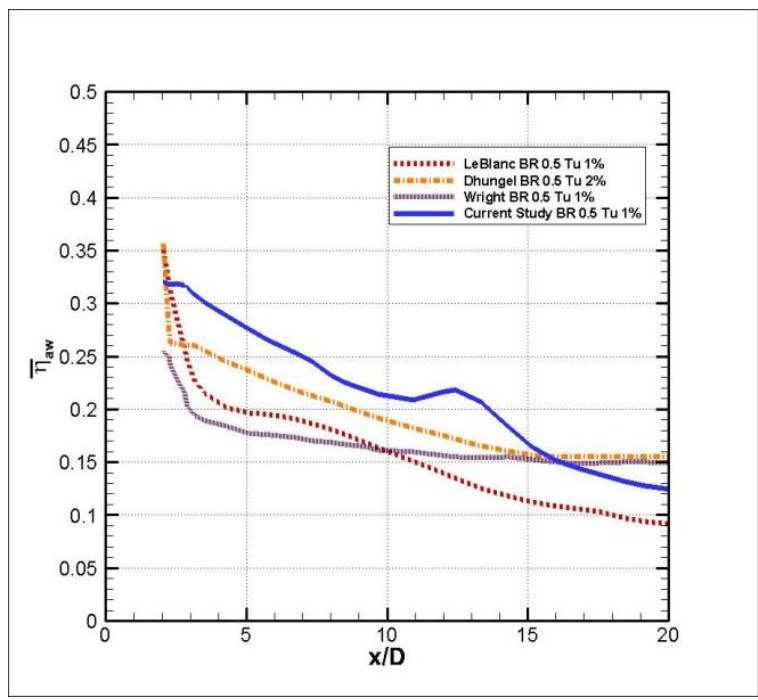

(a)

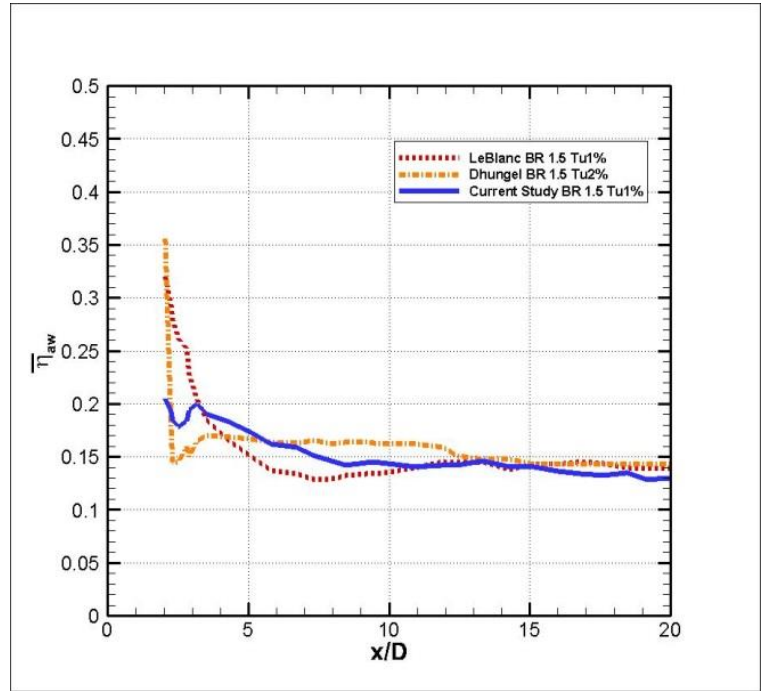

(c)

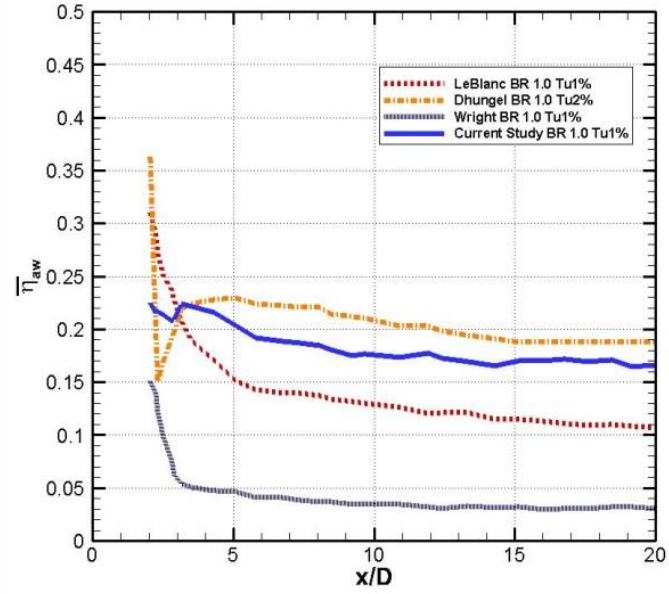

(b)

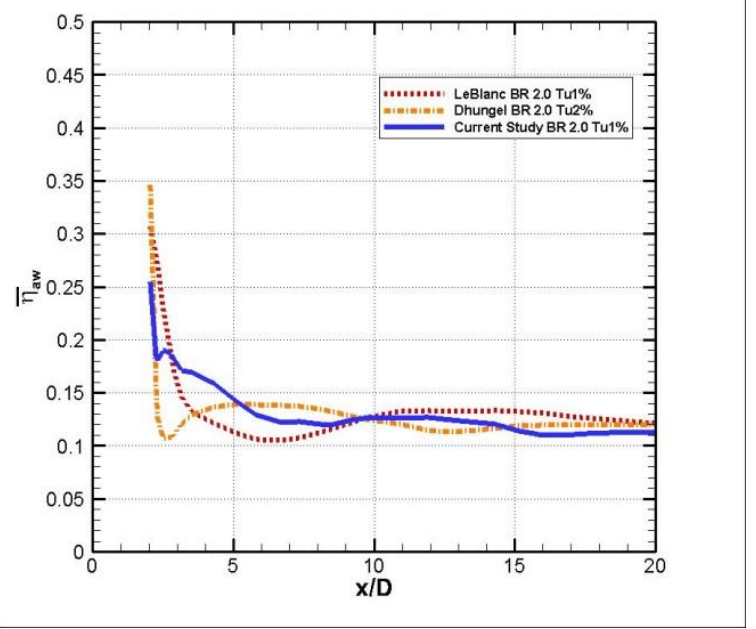

(d)

Figure 43: Span-averaged Film Cooling Effectiveness for Current and Past Baseline Studies [13, 29, 35]

It is also important to note that the leading edge of the coolant hole is at an $\mathrm{x} / \mathrm{D}$ location of 0 and the trailing edge of the coolant hole is the $\mathrm{x} / \mathrm{D}$ location of 2 for all data presented herein. Figure 44a visibly presents that increasing the blowing ratio for a straight hole decreases the span-averaged film cooling effectiveness beyond an $\mathrm{x} / \mathrm{D}=4$, and Figure $44 \mathrm{~b}$ displays that higher turbulence intensities will disperse the coolant jet laterally, producing a higher span-averaged effectiveness than at low turbulence intensity, which is especially noticeable at further locations downstream. Furthermore, from Figure 44b, at an x/D 
distance downstream of 10 the coolant holes, it may be interpreted that the coolant jets coalesce for the $7.5 \%$ turbulence case although this cannot be accurately proven without flow visualization. A higher span-averaged film cooling effectiveness at the downstream locations likely results from the coolant jet interaction.

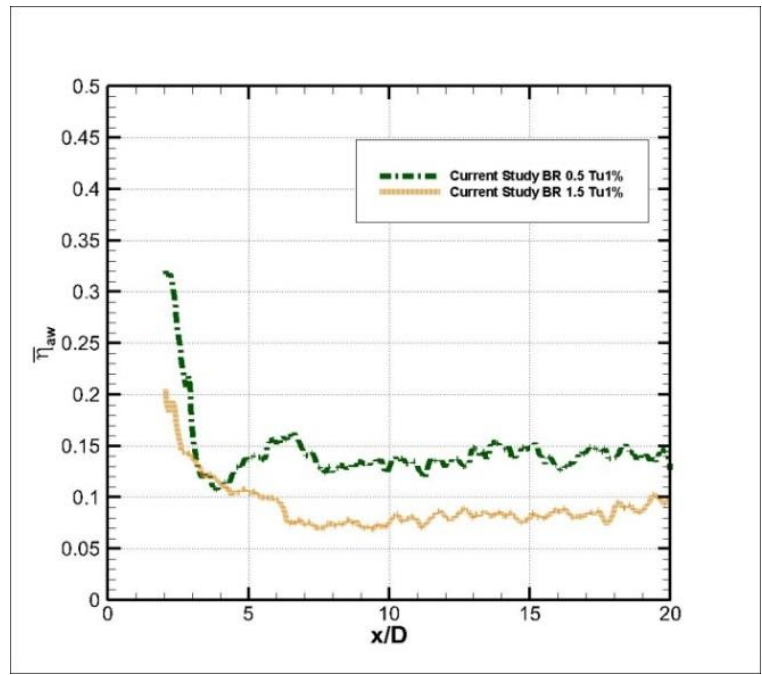

(a)

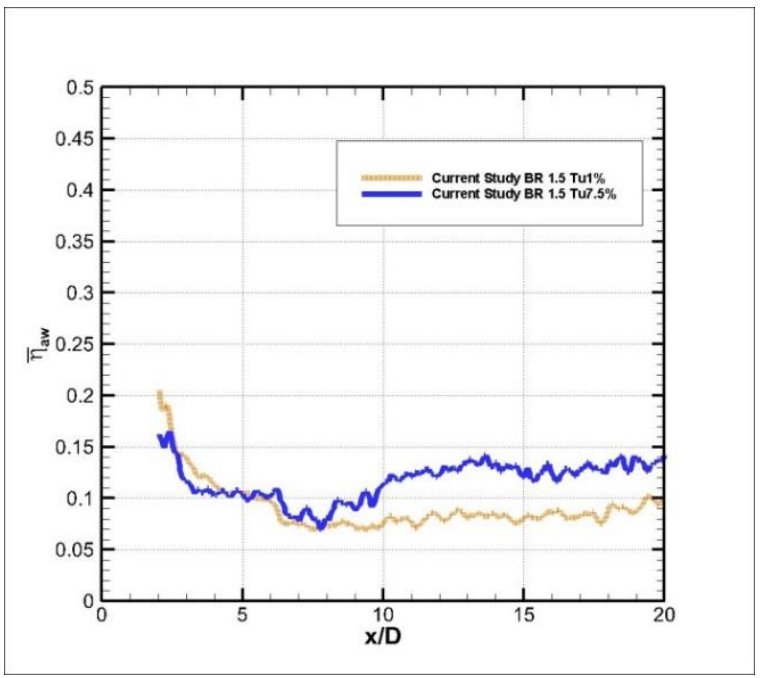

(b)

Figure 44: Span-averaged Film Cooling Effectiveness for Current Baseline Study

\subsubsection{CENTERLINE FILM COOLING EFFECTIVENESS}

The centerline data is pertinent because this allows one to determine how much jet lift off is occurring at the current test conditions. Additionally, the centerline film cooling effectiveness from the current study has been presented alongside a previous study in Figure 45. The trend of decreasing centerline effectiveness with increasing blowing ratio exists in both studies, and the values of the trend lines are similar as well. Figure 46 illustrates that as the turbulence intensity is increased the centerline effectiveness is decreased, likely due to the lateral expansion of the coolant jet. 


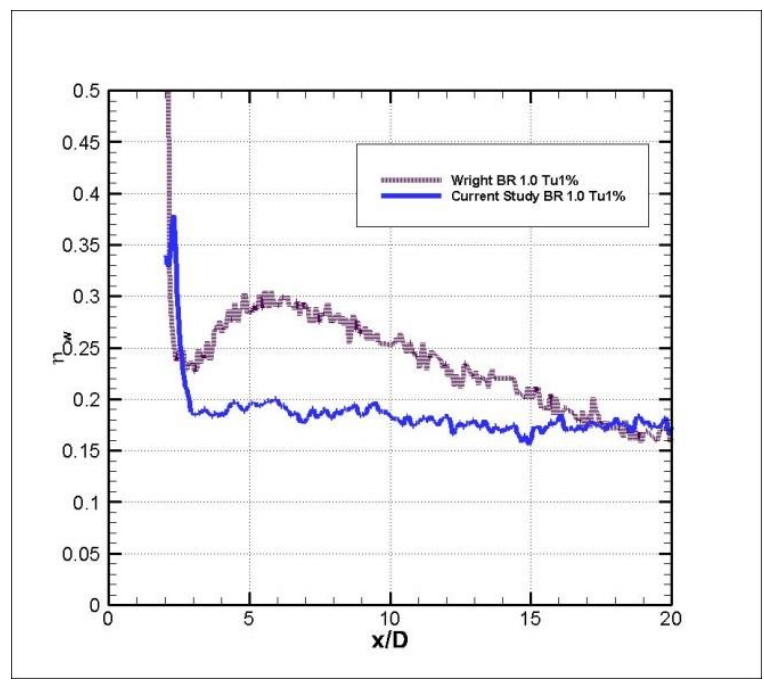

(a)

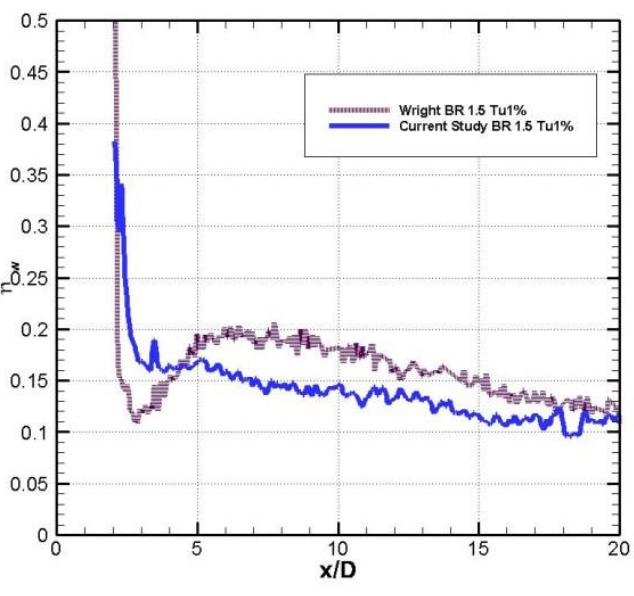

(b)

Figure 45: Centerline Film Cooling Effectiveness of Baseline Case of Current and Past Study [29]

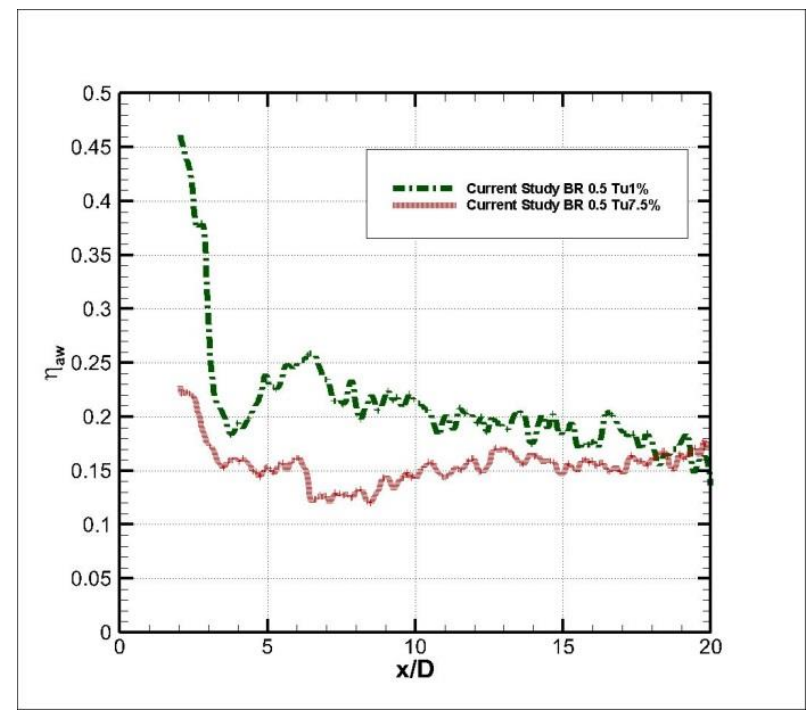

Figure 46: Centerline Film Cooling Effectiveness for Current Baseline Study

The results of the film cooling effectiveness for the baseline cases match the results of that published in previous literature. The span-averaged values also had the same trend of decreasing with increasing of the blowing ratio. Additionally, the span-averaged film cooling effectiveness increased with increased freestream turbulence intensity at high blowing ratio as was found in previous studies [24]. Finally, the centerline film cooling 
effectiveness decreased as the freestream turbulence intensity increased as was found in previous work [23].

\subsubsection{HEAT TRANSFER COEFFICIENT}

It is important to study the convective heat transfer coefficient because the goal with new film cooling hole geometries is to also reduce the convective heat transfer coefficient while increasing the film cooling effectiveness. The calculated heat transfer coefficient from the addition of coolant $\left(\mathrm{h}_{\mathrm{f}}\right)$ is found using the methodology outlined in Chapter 3 , and is non-dimensionalized by the convective heat transfer coefficient without cooling $\left(\mathrm{h}_{\mathrm{o}}\right)$. The convective heat transfer term without cooling was found by calculating the freestream Reynolds number and applying that to equation (4-1) in which the temperature passing over the plate imposes a uniform temperature and equation (4-2) which is the case of uniform surface heat flux. The average Nusselt number $(\mathrm{Nu})$ of these two values at each streamwise distance is found and then inserted into equation (4-3) to find the $h_{o}$ term. Here the Prandtl number $(\operatorname{Pr})$ is 0.709 and $(\mathrm{x})$ is the streamwise distance. In equation $(4-3),(\mathrm{k})$ is the thermal conductivity of air.

$$
\begin{gathered}
\mathrm{Nu}_{\mathrm{x}_{\text {temp }}}=0.0296 \operatorname{Re}_{\mathrm{x}}^{4 / 5} \operatorname{Pr}^{1 / 3} \\
\mathrm{Nu}_{\mathrm{x}_{\text {temp }}}=0.0308 \mathrm{Re}_{\mathrm{x}}^{4 / 5} \operatorname{Pr}^{1 / 3} \\
\mathrm{~h}_{\mathrm{o}_{\mathrm{x}}}=\frac{\mathrm{Nu}_{\mathrm{x}} \mathrm{k}}{\mathrm{x}}
\end{gathered}
$$

Presented in Figure 47 are the span-averaged and centerline dimensionless convective heat transfer coefficients of the current study compared with a previous study conducted by Russin et al. [51]. The data of the current study are comparable to the previously published research. 


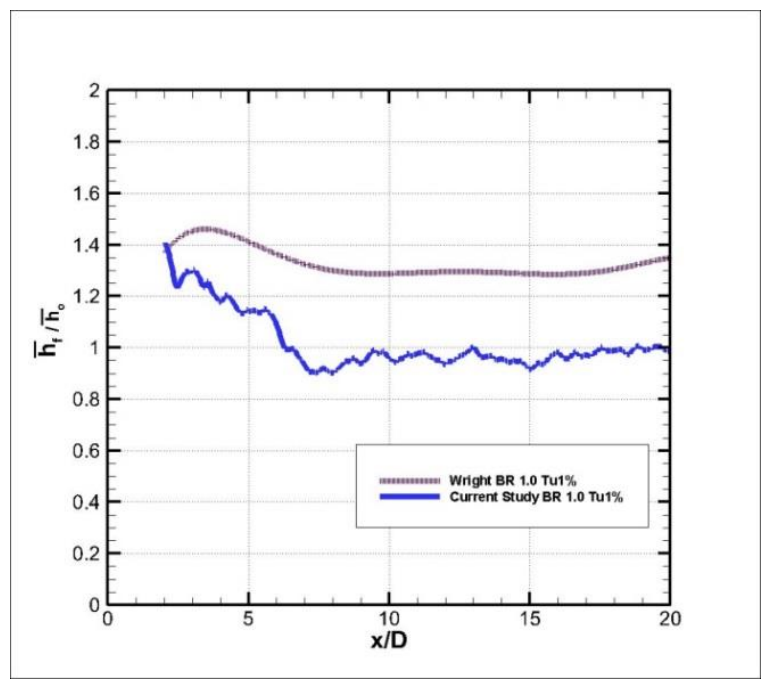

(a)

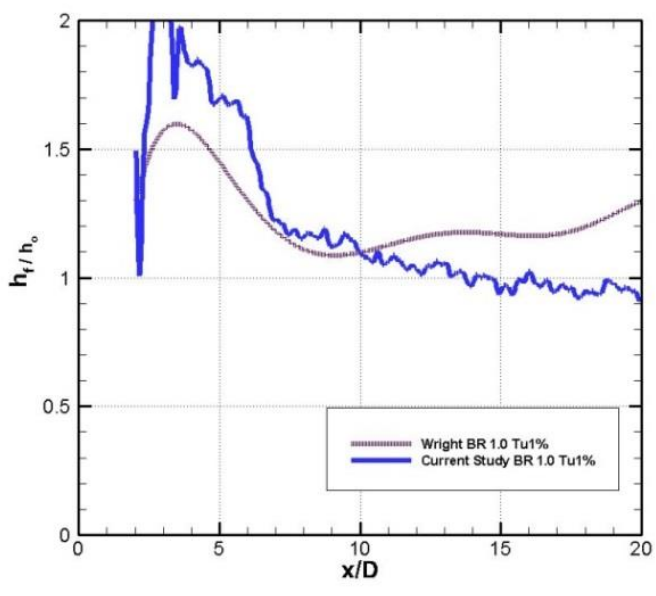

(b)

Figure 47: Dimensionless Heat Transfer Coefficient of Baseline Case of Current and Past Study [51]

\subsection{ANTI-VORTEX HOLE (AVH) RESULTS}

The current work is mainly concerned with investigating the effects of elevated freestream turbulence intensity upon an $\mathrm{AVH}$ configuration for a range of blowing ratios. A major concern with the AVH will be how the secondary jets interact with the main coolant jet in counteracting the CRV pair, and this phenomenon has not been investigated in previous published literature. Presented here, as an easily accessible reference, Figure 48 and Table 3 again present the AVH geometry configuration that was used during the current study which was introduced in Chapter 1. 


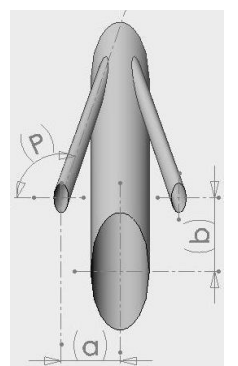

Top View

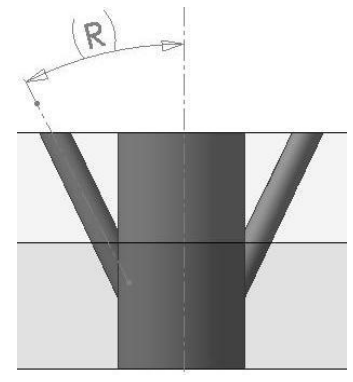

Front View

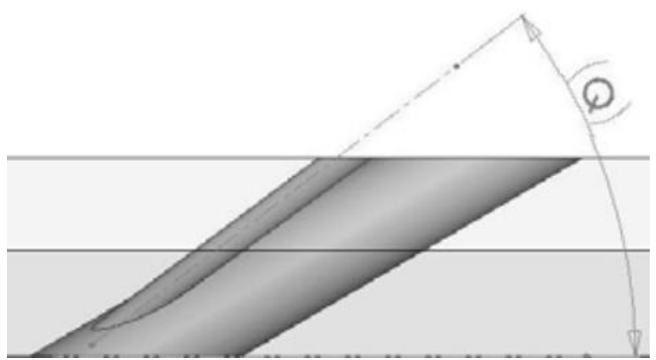

Side View

Figure 48: Three View Drawing of Anti-Vortex Hole Geometry

Table 3: Geometric Parameters of Current AVH Geometry

\begin{tabular}{|c|c|}
\hline $\mathbf{D}_{\text {main }}(\mathbf{c m})$ & 1.27 \\
\hline $\mathbf{D}_{\text {secondary }}(\mathbf{c m})$ & 0.63 \\
\hline $\mathbf{a}(\mathbf{c m})$ & 1.42 \\
\hline $\mathbf{b}(\mathbf{c m})$ & -0.56 \\
\hline $\mathbf{P}\left({ }^{\mathbf{o}}\right)$ & 105.67 \\
\hline $\mathbf{Q}\left(^{\mathbf{o}}\right)$ & 26.41 \\
\hline $\mathbf{R}\left({ }^{\mathbf{0}}\right)$ & 27.91 \\
\hline
\end{tabular}

Table 4 presents the test matrix of the experiments performed. Three different freestream turbulence intensities of $1,7.5$, and $11.7 \%$ with four different blowing ratios of 0.5, 1.0, 1.5, and 2.0 were studied. Each experiment was repeated four separate times to ensure the accuracy and precision of the findings. 
Table 4: Anti-vortex Hole Test Matrix

\begin{tabular}{|c|c|c|}
\hline Experiment & Blowing Ratio (BR) & Turbulence Intensity (Tu) \\
\hline A & 0.5 & $1 \%$ \\
\hline B & 1.0 & $1 \%$ \\
\hline C & 1.5 & $1 \%$ \\
\hline D & 2.0 & $1 \%$ \\
\hline E & 0.5 & $7.5 \%$ \\
\hline F & 1.0 & $7.5 \%$ \\
\hline G & 1.5 & $7.5 \%$ \\
\hline I & 2.0 & $7.5 \%$ \\
\hline J & 0.5 & $11.7 \%$ \\
\hline K & 1.0 & $11.7 \%$ \\
\hline L & 1.5 & $11.7 \%$ \\
\hline
\end{tabular}

\subsubsection{FILM COOLING EFFECTIVENESS}

\subsubsection{ANTI-VORTEX HOLE SURFACE CONTOUR PLOTS}

Contour plots allow for a general idea of the flow to be visualized while showing specific locations of increased or diminished cooling which would not be as easily discernable through the span-averaged and centerline plots. Figure 49 compares contour plots of film cooling effectiveness for varying blowing ratio at constant freestream turbulence intensity and Figure 50 compares the contour plots of constant blowing ratio with varying freestream turbulence intensity, again for a non-dimensionalized streamwise distance (x/D) of 27. 


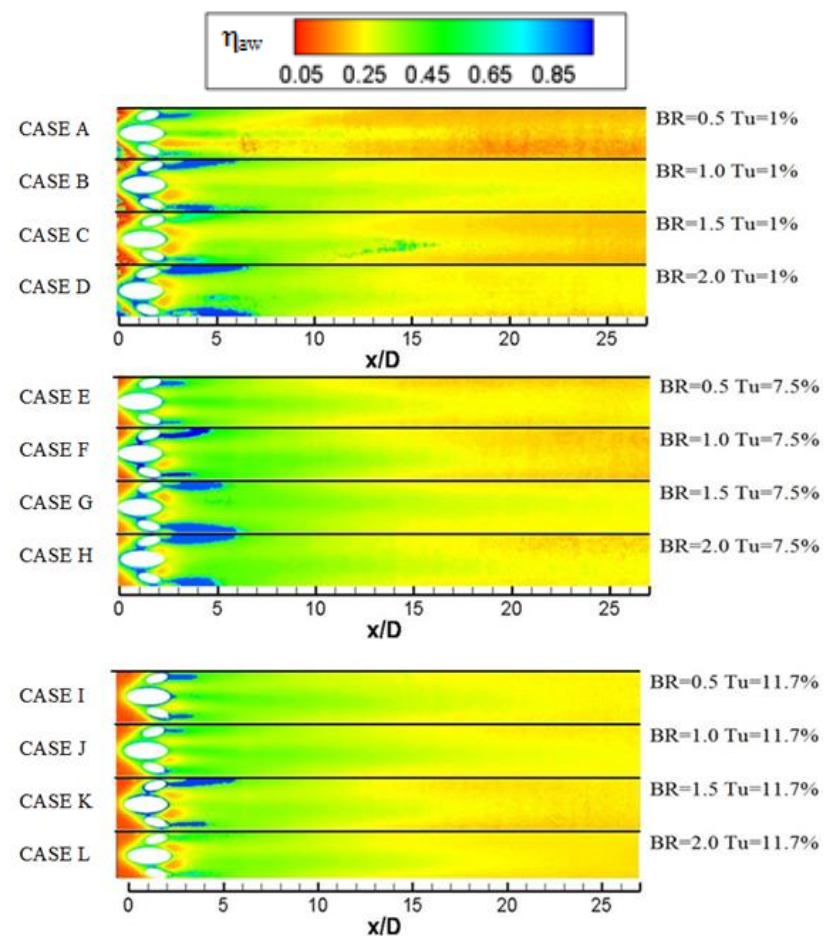

Figure 49: Film Cooling Effectiveness Contour Plots of Varying Blowing Ratios at a Constant Turbulence Intensity

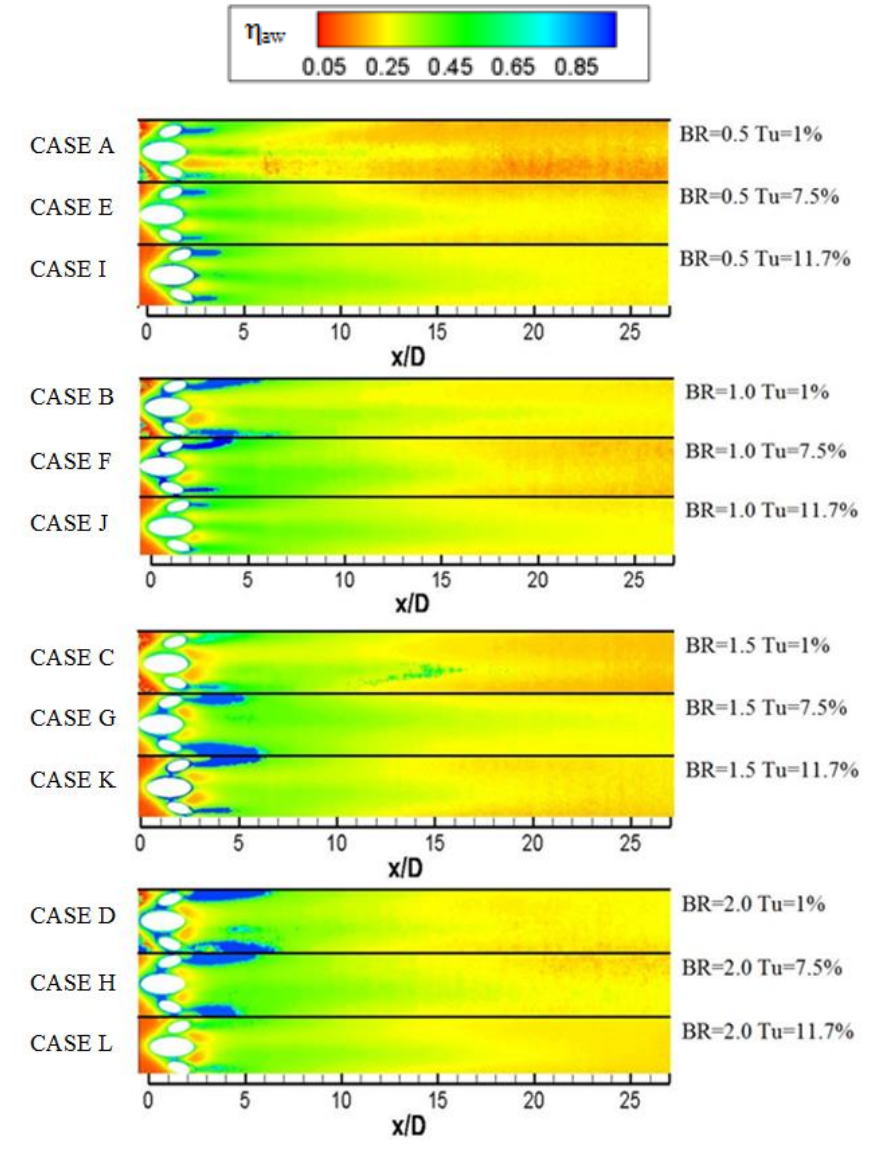

Figure 50: Film Cooling Effectiveness Contour Plots of Varying Turbulence Intensity with Constant Blowing Ratio 
For the low turbulence case of $1 \%$ it is clearly visible through the use of the contour plots that the film cooling effectiveness is enhanced with an increase in blowing ratio not only in the lateral direction, but the streamwise direction as well. Distinct cooling is prevalent out of the two secondary holes on the midline between the main holes and are actually the regions of highest effectiveness which is not the case in the near hole region for straight holes at higher blowing ratios. This strongly suggests that jet lift off is not prevalent out of the secondary holes. At higher turbulence, the lateral spreading of the coolant jets is improved over that in the low turbulence cases with it appearing that the optimum coverage occurs for the $7.5 \%$ cases for blowing ratios of 1.5 and 2.0 respectively. At the higher blowing ratios of 1.5 and 2.0 it is easily discernable that the film cooling effectiveness is much higher downstream for the $7.5 \%$ turbulence case than it is for the $1 \%$ and $11.7 \%$ cases. At high blowing ratios, the secondary holes are not as effective on the midline for the high turbulence case of $11.7 \%$ as they are for the $7.5 \%$ case. At low blowing ratio, span-averaged film cooling effectiveness is noticeably improved with increasing freestream turbulence intensity as the lateral spreading appears to be improved along with the streamwise performance also improving.

\subsubsection{ANTI-VORTEX HOLE SPAN-AVERAGED FILM COOLING EFFECTIVENESS}

The span-averaged film cooling effectiveness is the best gauge in determining the overall performance of a cooling hole geometry and as such will be discussed in this section. The span-averaged film cooling effectiveness values at each of the turbulence intensities for all four blowing ratios are presented in Figure 51. The span-averaged film cooling effectiveness at turbulence intensities of 1 and $7.5 \%$ are the highest at a blowing ratio of 2.0 beyond a non-dimensional streamwise distance of 5, but appear to be highest 
for blowing ratios of 1.0 and 1.5 at the highest freestream turbulence intensity of $11.7 \%$. The span-averaged effectiveness at a blowing ratio of 0.5 is lowest in all cases except for the 2-3 hole diameters directly behind the trailing edge of the cooling hole. The low blowing ratio cases for a freestream turbulence intensity of $7.5 \%$ were much less effective compared to the high blowing ratio cases beyond an $\mathrm{x} / \mathrm{D}=5$ downstream.

Presented in Figure 52 is the span-averaged film cooling effectiveness at a specific blowing ratio for all three freestream turbulence intensities. For blowing ratios of 1.5 and 2.0 it can be seen that the highest span-averaged effectiveness occurs at a freestream turbulence intensity of $7.5 \%$. At a blowing ratio of 0.5 , the low turbulence case of $1 \%$ has a higher span-averaged effectiveness than the higher turbulence cases. 


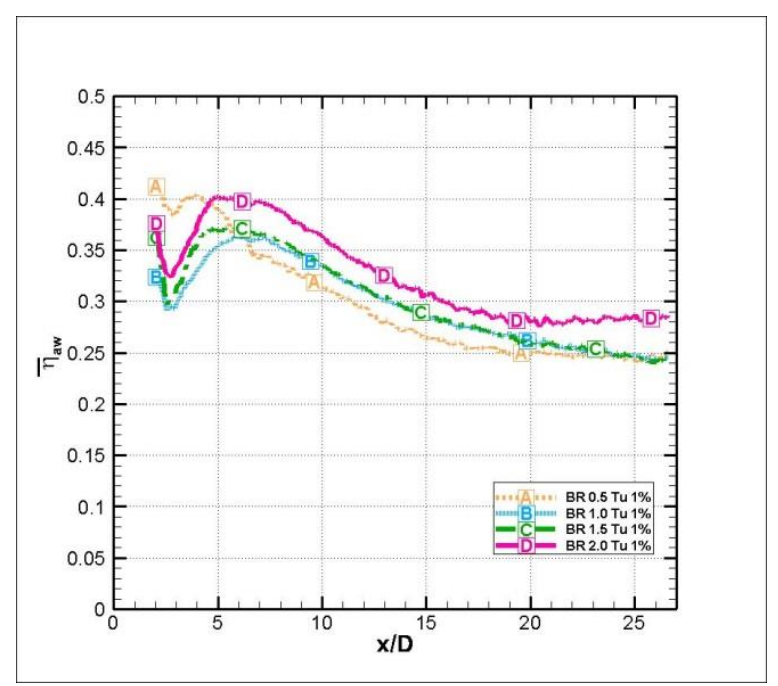

(a)

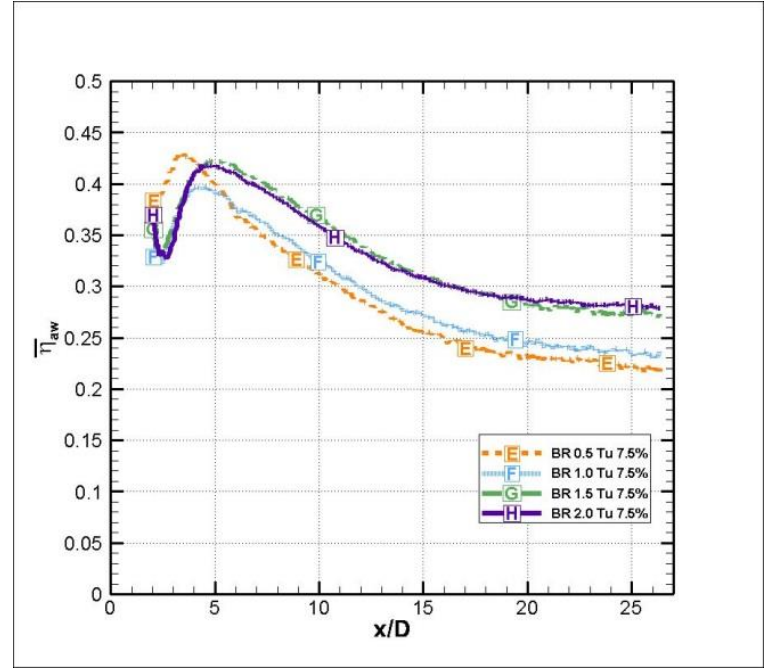

(b)

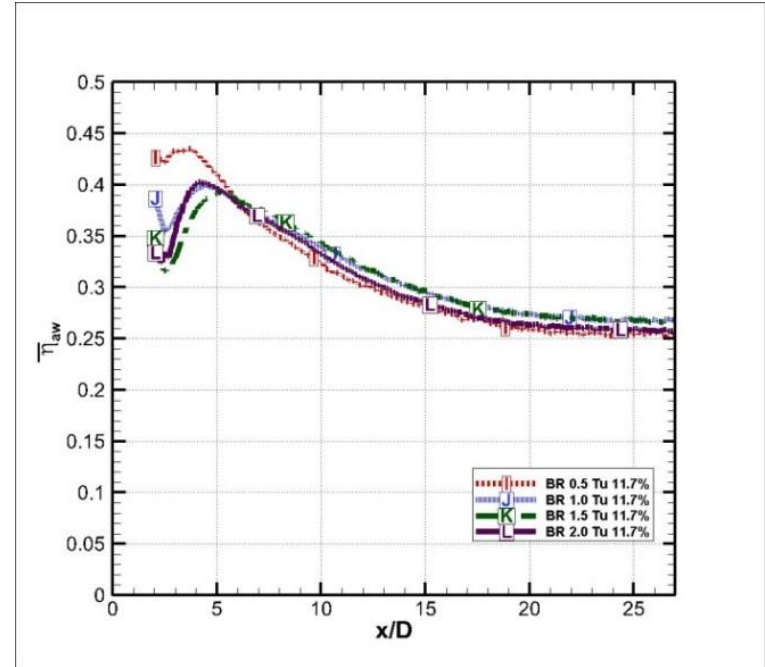

(c)

Figure 51: Span-averaged Film Cooling Effectiveness at Varying Blowing Ratio 


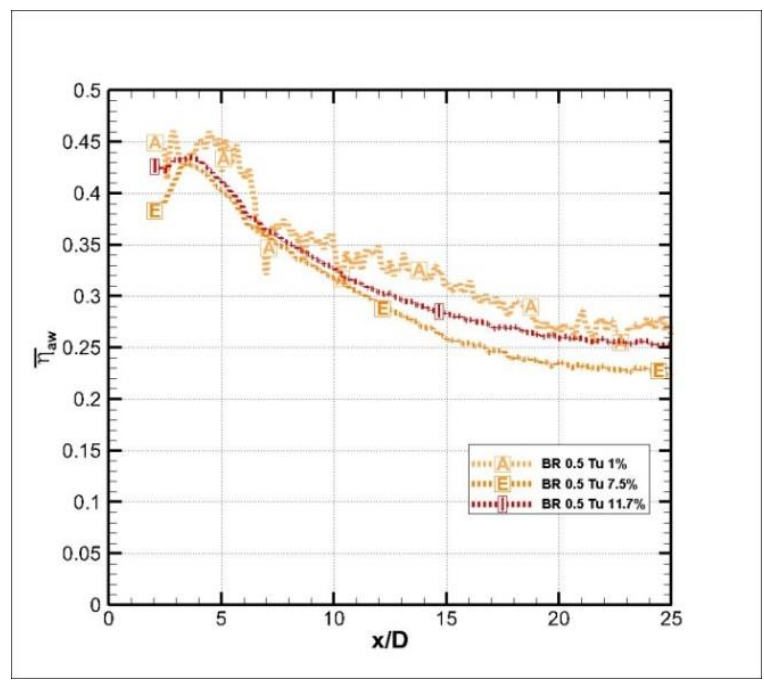

(a)

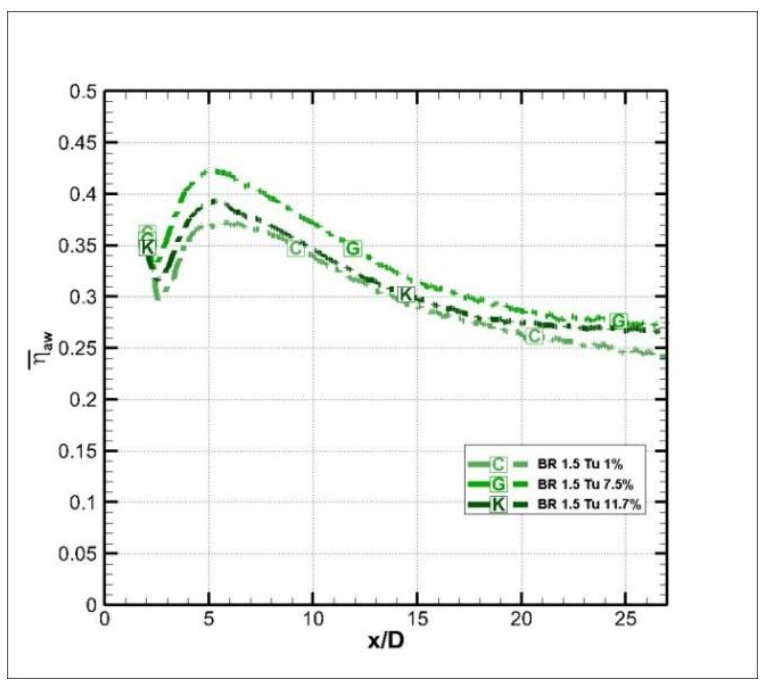

(c)

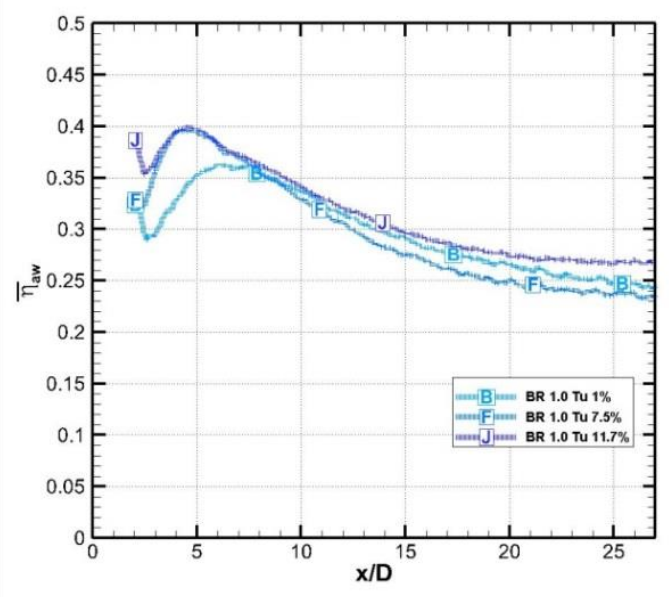

(b)

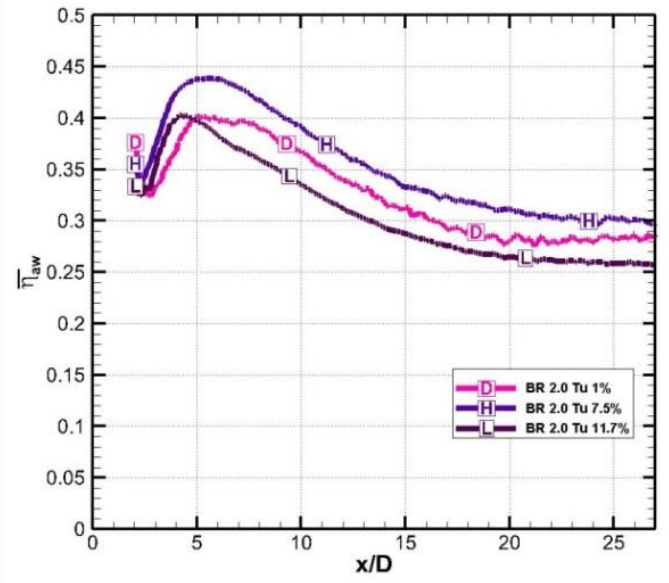

(d)

Figure 52: Span-averaged Film Cooling Effectiveness at Varying Turbulence Intensity

\subsubsection{ANTI-VORTEX HOLE CENTERLINE FILM COOLING EFFECTTIVENESS}

The centerline film cooling effectiveness plots are valuable because this allows for a visual way of determining if jet lift off is occurring at the given conditions. Figure 53 compares the centerline film cooling effectiveness values for varying blowing ratios at a given turbulence intensity. Figure 54 compares the centerline film cooling effectiveness for a constant blowing ratio with varying freestream turbulence intensity. 


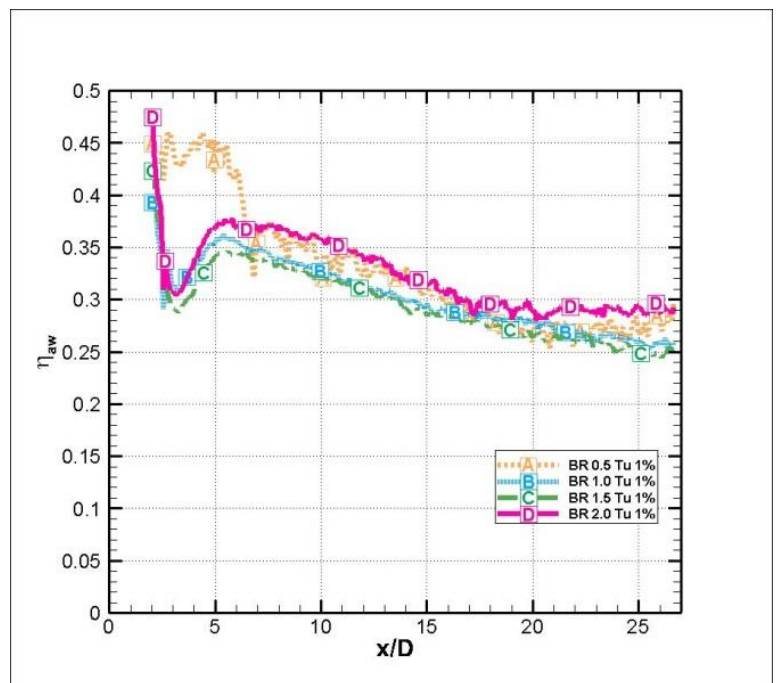

(a)

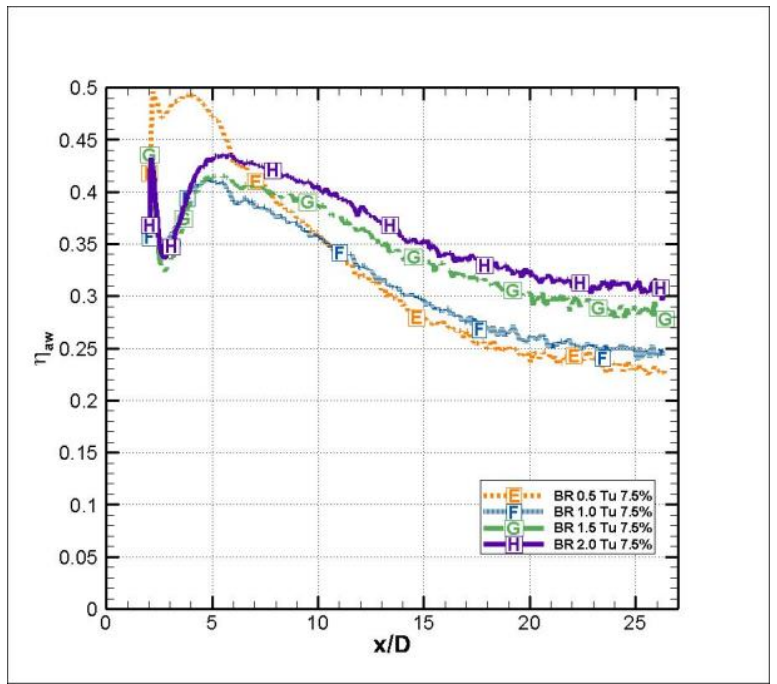

(b)

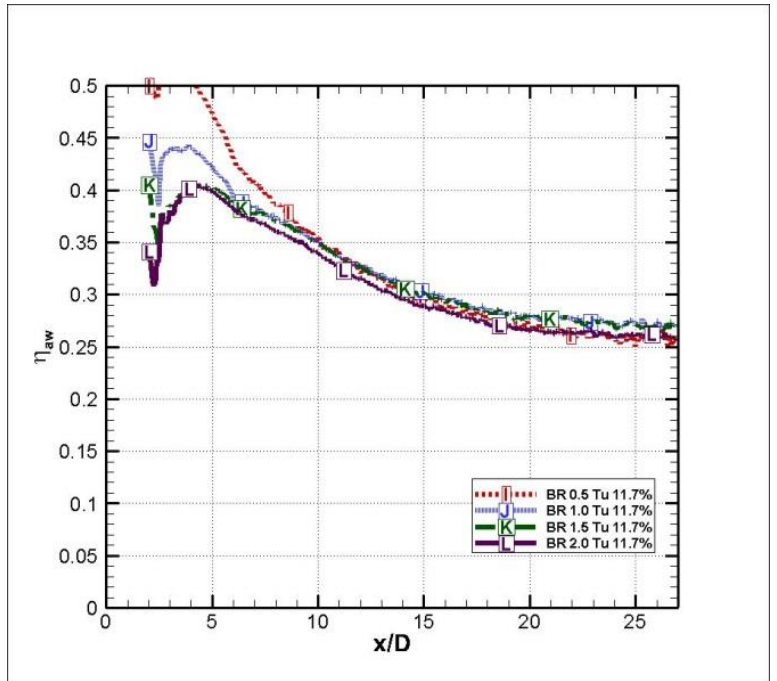

(c)

Figure 53: Centerline Film Cooling Effectiveness for Varying Blowing Ratio with Constant Turbulence Intensity 


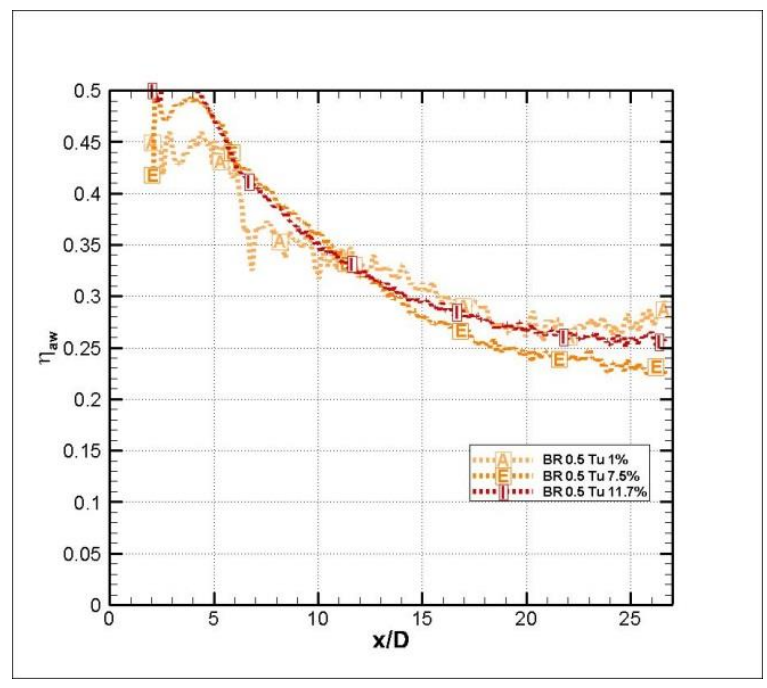

(a)

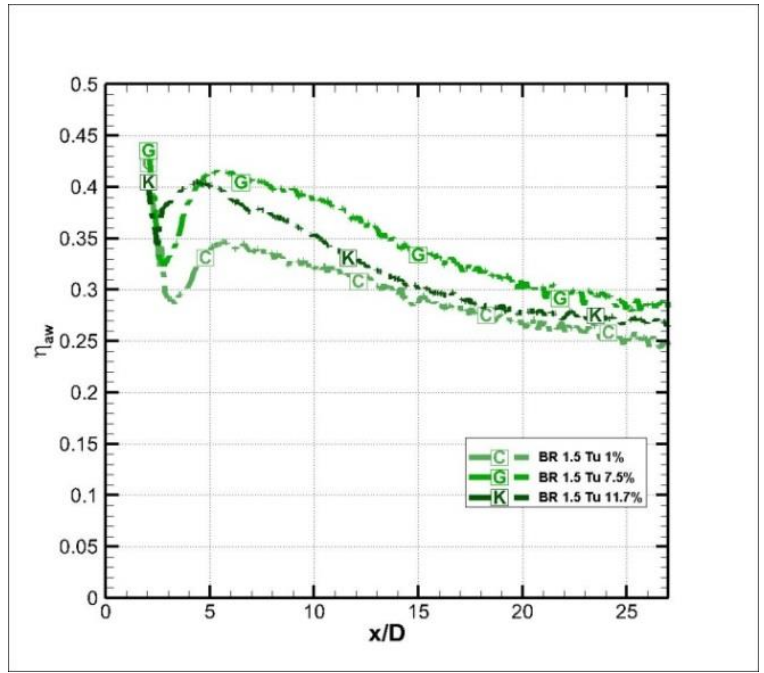

(c)

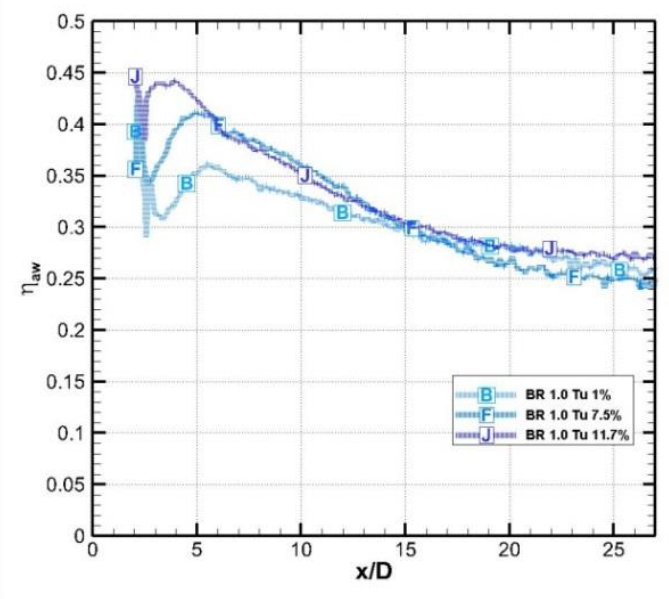

(b)

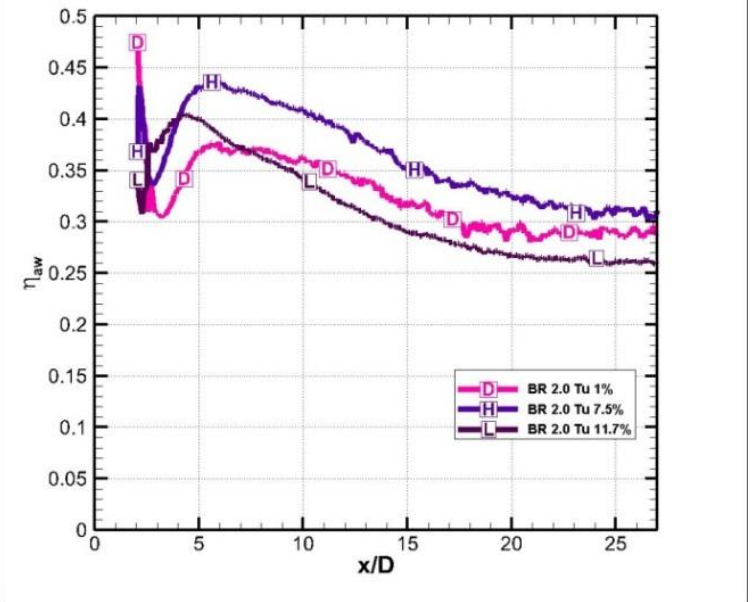

(d)

Figure 54: Centerline Film Cooling Effectiveness at Varying Turbulence Intensity

The centerline effectiveness improves with increased blowing ratio for the 1 and $7.5 \%$ turbulence cases. This is the opposite of that experienced by the straight hole design, and is a highly desired result for real applications, showing jet lift off reduction. The highest effectiveness occurs at the turbulence intensity of $7.5 \%$ and a blowing ratio of 2.0. This is possible because at this turbulence intensity the coolant jets spread more laterally than the $1 \%$ case and the secondary hole CRV pairs are capable of still counteracting the main hole CRV pair. 
The span-averaged effectiveness and centerline effectiveness remain rather different between different blowing ratios for the two lower turbulence cases, but at a turbulence level of $11.7 \%$ the effectiveness trends to similar values past an $\mathrm{x} / \mathrm{D}$ location of 5 . At a turbulence intensity of $11.7 \%$ the span-averaged effectiveness is lower than the $7.5 \%$ case most noticeably at blowing ratios of 1.5 and 2.0 , which is most likely due to the enhanced mixing of the freestream with the coolant and the CRV pairs of the secondary holes being weakened making them less capable of cancelling out the CRV of the main hole. Figure 54 displays the centerline data and at higher blowing ratios jet lift off is still prevalent, but is still the highest at a turbulence intensity of $7.5 \%$. The span-averaged and centerline film cooling effectiveness reaches a maximum at approximately 5 hole diameters downstream.

\subsubsection{ANTI-VORTEX HOLE AREA-AVERAGED FILM COOLING EFFECTIVENESS}

The area-averaged results give a general idea of how the geometry is performing, but do not give a clear idea of what is truly happening in specific regions. The area-averaged film cooling effectiveness is presented in Table 5 for each blowing ratio and freestream turbulence intensity. At each of the turbulence intensities the general trend is that as the blowing ratio is increased, the film cooling effectiveness is also increased. The two optimum cases were at a blowing ratio of 2.0 and a blowing ratio of 1.5 for the $7.5 \%$ turbulence cases. The two highest turbulence cases generally outperformed the same blowing ratio cases for the low turbulence intensity which is the desired result of the test. The lowest blowing ratio case at the lowest freestream turbulence intensity also has an area-averaged effectiveness close to the two optimum cases. This is largely due to the high effectiveness experienced in the near hole region where jet lift off is not an issue for the low blowing ratio of 0.5 . 
Table 5: Area-averaged Film Cooling Effectiveness

\begin{tabular}{|c|c|c|c|}
\hline Case & Tu 1\% & Tu 7.5\% & Tu 11.7\% \\
\hline BR 0.5 & 0.328 & 0.2884 & 0.308 \\
\hline BR 1.0 & 0.296 & 0.2943 & 0.314 \\
\hline BR 1.5 & 0.2997 & 0.3301 & 0.3119 \\
\hline BR 2.0 & 0.3248 & 0.3512 & 0.3288 \\
\hline
\end{tabular}

\subsubsection{COMPARISONS TO PREVIOUS RESEARCH}

Dhungel et al. [13] conducted their experimental study at a freestream Reynolds number based on cooling hole diameter of $\approx 9700$ which is near the Reynolds number of the current study $(\approx 10,000)$. Their study also included $30^{\circ}$ inclined holes with hole pitch of three hole diameters and hole diameters of $1 / 2$ " with blowing ratios of $0.5,1.0,1.5$, and 2.0 which are all identical to the current study, but had a freestream turbulence intensity of $2 \%$ rather than the $1 \%$ freestream turbulence intensity in the current study. Their span-averaged results are presented in Figure 55 for the same exact AVH geometry as the current study. The results show that the highest performing cases at low turbulence intensity are at blowing ratios of 1.0 and 1.5 and the lowest performing case is for a low blowing ratio of 0.5 . These results agree with the current study in the fact that the blowing ratio cases of 1.0 and 1.5 are practically the same and the lowest performing case is the blowing ratio of 0.5 . However, the highest performing case in the current study at low freestream turbulence intensity was the blowing ratio case of 2.0. 


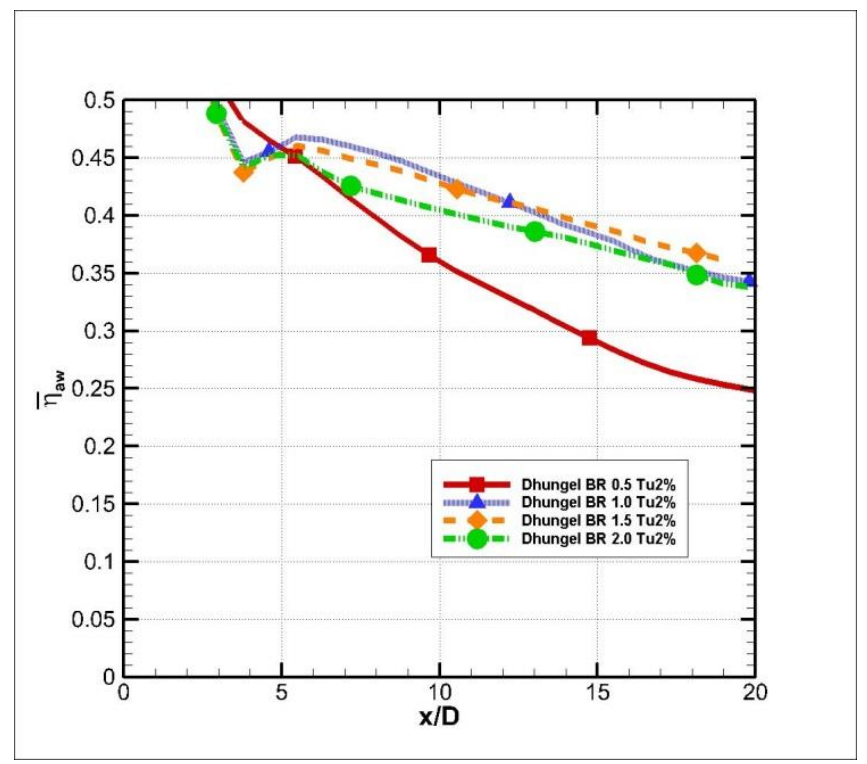

Figure 55: Span-Averaged Film Cooling Effectiveness of Anti-Vortex Hole [13]

Hussien et al. [14] numerically determined the span-averaged film cooling effectiveness for a near exact anti-vortex hole geometry to the one in the current experimental investigation. To numerically solve for the film cooling effectiveness a k- $\varepsilon$ model in a FLUENT commercial code was used to produce results for multiple blowing ratios at a density ratio of 1.0 and turbulence intensity of $1 \%$. Table 6 compares the differences in the AVH geometries and also lists the flow conditions. The results of their data are presented with the cases of the current research in Figure 56.

Table 6: Current Study and Previous Numerical Results

\begin{tabular}{|c|c|c|}
\cline { 2 - 3 } \multicolumn{1}{c|}{} & Current Study & Hussien et al. [14] \\
\hline $\mathbf{D}_{\text {main }}(\mathbf{c m})$ & 1.27 & 1.00 \\
\hline $\mathbf{D}_{\text {secondary }}(\mathbf{c m})$ & 0.63 & 0.50 \\
\hline $\mathbf{a}(\mathbf{c m})$ & 1.42 & 1.00 \\
\hline $\mathbf{b}(\mathbf{c m})$ & -0.56 & 0.00 \\
\hline $\mathbf{Q}\left(^{\mathbf{0}}\right)$ & 26.41 & 30 \\
\hline $\mathbf{R}\left(\mathbf{o}^{\mathbf{}}\right)$ & 27.91 & 30 \\
\hline $\mathbf{L} / \mathbf{D}_{\text {main }}$ & 4 & 4 \\
\hline $\mathbf{D R}$ & 1.05 & 1.0 \\
\hline $\mathbf{T u}(\%)$ & $1 \%$ & $1 \%$ \\
\hline
\end{tabular}




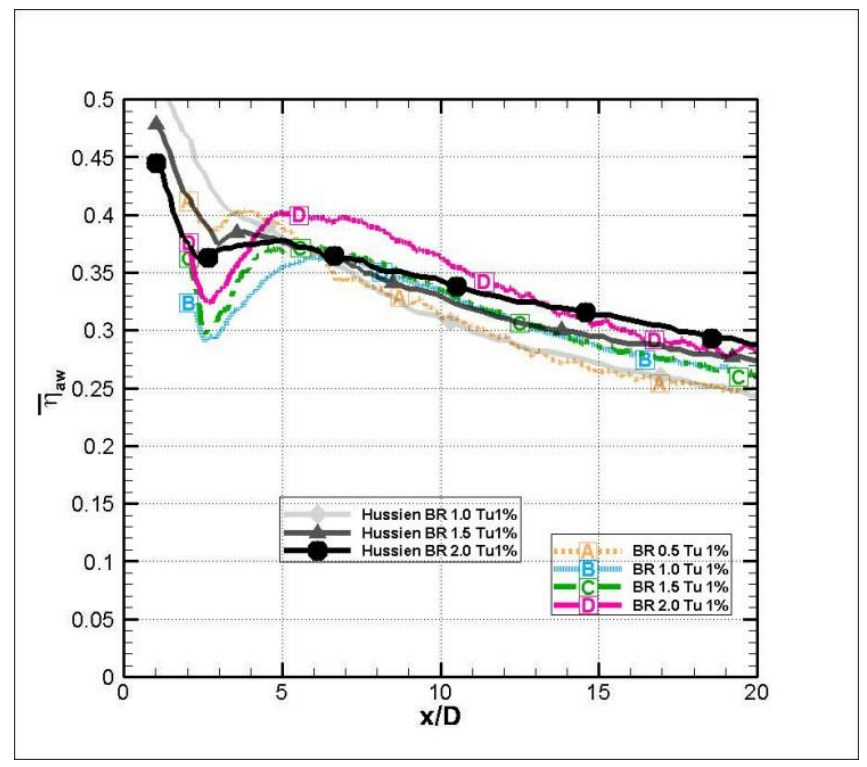

Figure 56: Span-averaged Film Cooling Effectiveness of Current Study and Previous Numerical Study [14]

The results of both studies show the same trends of an increased span-averaged effectiveness as the blowing ratio is increased past the first 5 hole diameters. Both studies also show that a maximum cooling effectiveness occurs at approximately 5 hole diameters downstream, in addition to similar values for the film cooling effectiveness across the entire streamwise direction. Moreover, the case for a blowing ratio of 1.5 is nearly identical beyond the first 5 hole diameters for both studies.

\subsubsection{HEAT TRANSFER COEFFICIENT}

Again, with the creation of new film cooling hole geometries it is important to reduce the convective heat transfer coefficient, and with that, deduce where hotspot formations from entrained freestream flow are prevalent. Heat transfer to a film cooling airfoil is defined in equation (4-4) where (q") is the heat flux per unit area.

$$
\mathrm{q}^{\prime \prime}=\mathrm{h}_{\mathrm{f}}\left(\mathrm{T}_{\mathrm{aw}}-\mathrm{T}_{\mathrm{w}}\right)
$$

Sen et al. [52] introduced a parameter known as net heat flux reduction (NHFR) which determines the net benefit resulting from film cooling. The NHFR parameter is defined in 
equation (4-5). This term serves to quantify the relationship between the non-dimensional convective heat transfer coefficient and the adiabatic film cooling effectiveness.

$$
\text { NHFR }=1-\frac{\mathrm{q}^{\prime \prime}}{\mathrm{q}_{\mathrm{o}}{ }^{\prime \prime}}=\frac{\mathrm{h}_{\mathrm{f}}\left(\mathrm{T}_{\mathrm{aw}}-\mathrm{T}_{\mathrm{w}}\right)}{\mathrm{h}_{\mathrm{o}}\left(\mathrm{T}_{\infty}-\mathrm{T}_{\mathrm{w}}\right)}
$$

A dimensionless temperature is defined in equation (4-6) and is used to reduce the NHFR equation to its most advantageous form, as presented in equation (4-7). The dimensionless temperature $(\theta)$ of 1.6 is used and is considered to be representative of engine conditions [11].

$$
\begin{gathered}
\theta=\frac{\mathrm{T}_{\infty}-\mathrm{T}_{\mathrm{c}}}{\mathrm{T}_{\infty}-\mathrm{T}_{\mathrm{w}}}=1.6 \\
\mathrm{NHFR}=1-\frac{\mathrm{h}_{\mathrm{f}}}{\mathrm{h}_{\mathrm{o}}}\left(1-\eta_{\mathrm{aw}} \theta\right)
\end{gathered}
$$

The NHFR can be negative in areas where the film cooling effectiveness is especially high and the non-dimensional convective heat transfer coefficient is also high, such as the region directly at the trailing edge of the coolant hole $(x / D=2)$. The typical range however for NHFR is typically between zero (0) to one (1). The NHFR data will be presented along with span-averaged heat transfer coefficient data.

\subsubsection{ANTI-VORTEX HOLE SPAN-AVERAGED HEAT TRANSFER COEFFICIENT}

The span-averaged heat transfer coefficient in dimensionless form is presented in Figure 57 at constant blowing ratio for varying freestream turbulence intensity. The spanaveraged non-dimensionalized heat transfer coefficient for varying blowing ratio at constant freestream turbulence intensity is presented in Figure 58. 


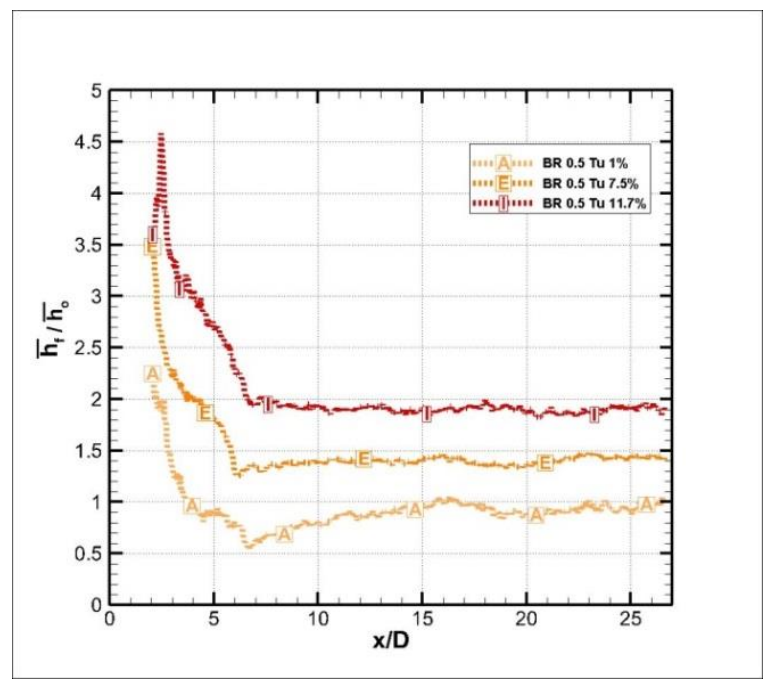

(a)

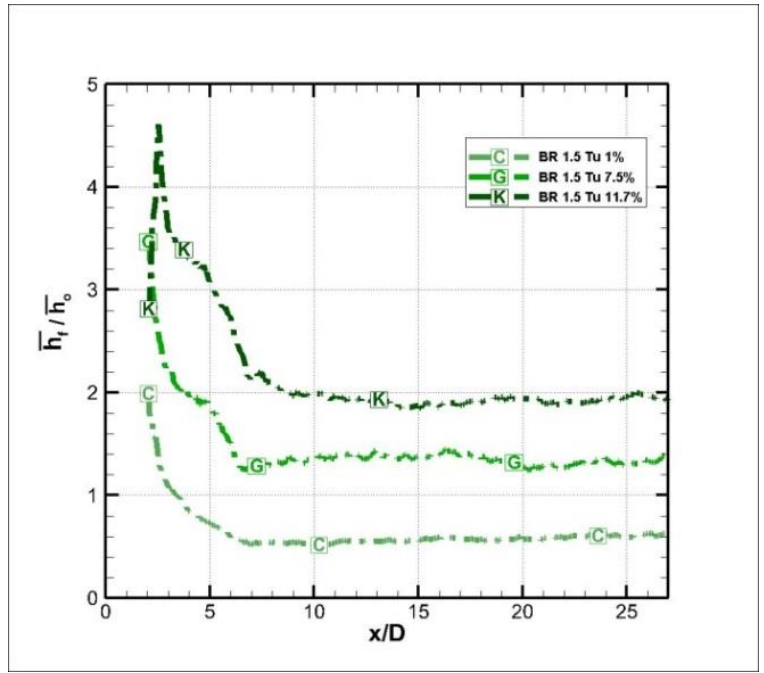

(c)

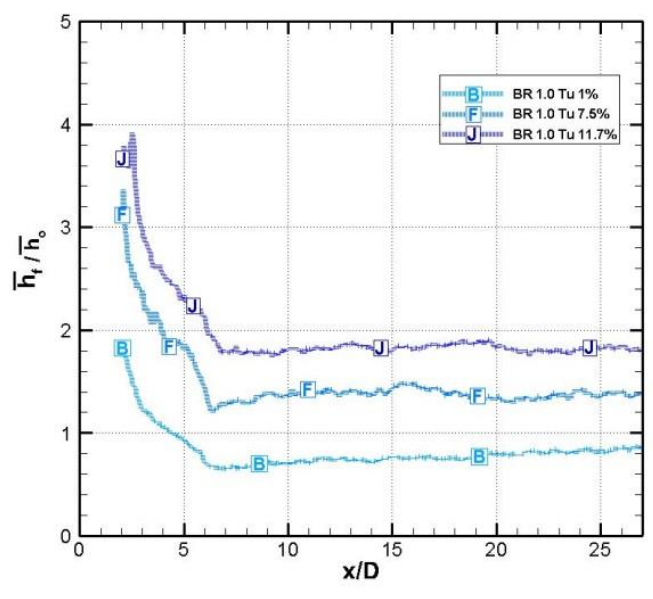

(b)

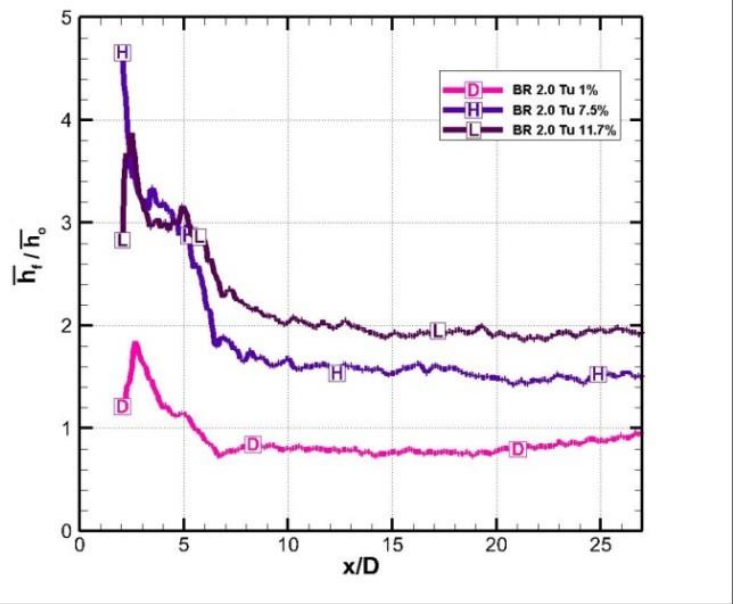

(d)

Figure 57: Span-averaged Dimensionless Heat Transfer Coefficient for Varying Turbulence Intensity 


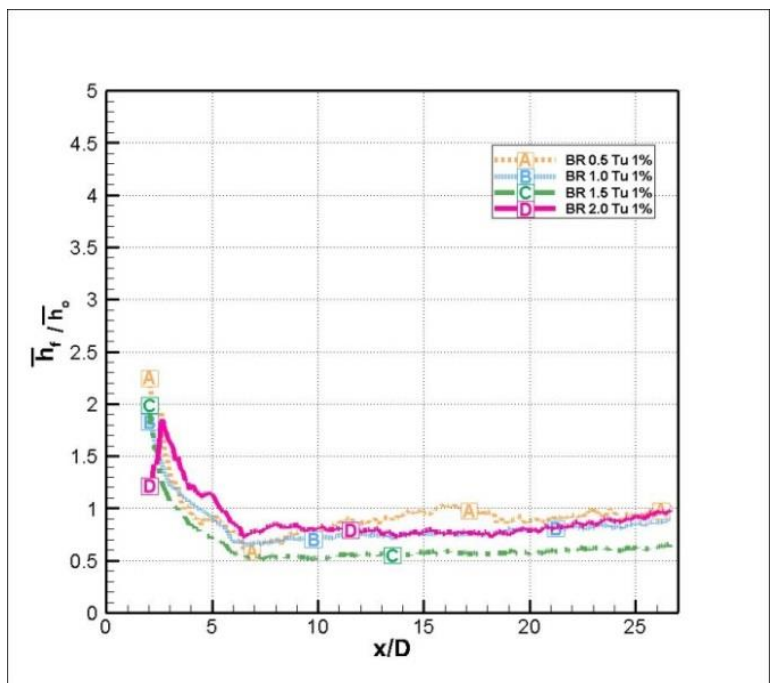

(a)

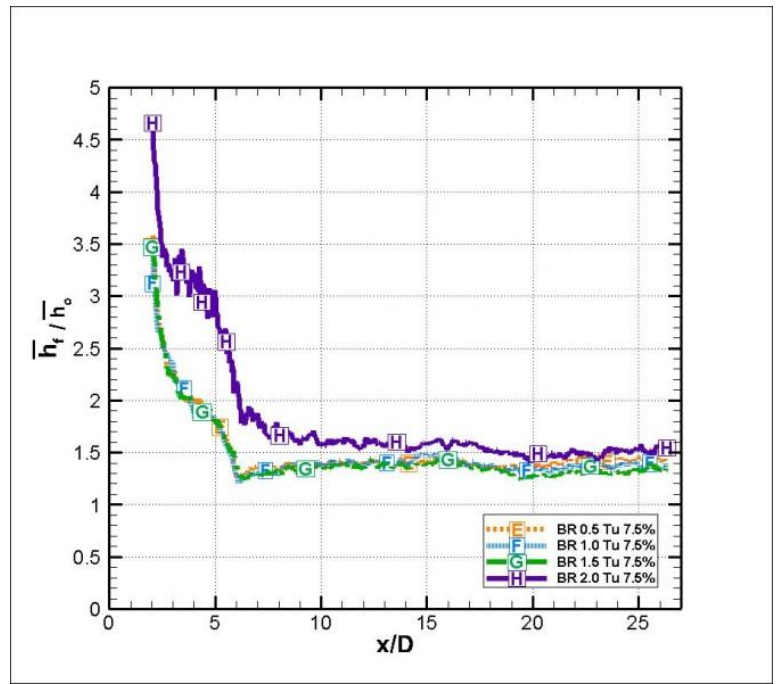

(b)

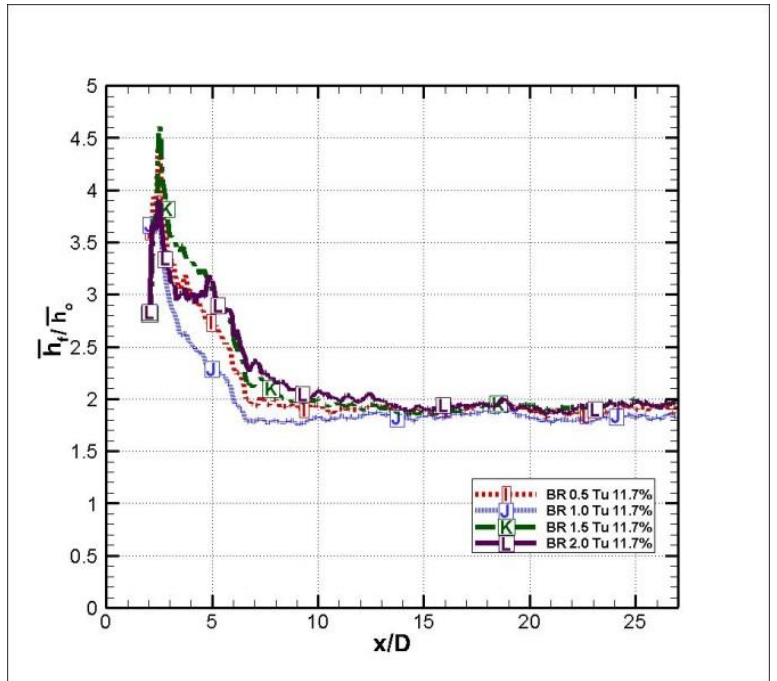

(c)

Figure 58: Span-averaged Dimensionless Heat Transfer Coefficient at Varying Blowing Ratio 
At freestream turbulence intensities of $7.5 \%$ and $11.7 \%$, as the blowing ratio is increased the heat transfer is also increased. This is expected because as the freestream moves around the coolant jets, which primarily occurs in the first 7 hole diameters downstream, the local turbulence intensity is increased as the coolant jets and freestream mix resulting in a higher heat transfer coefficient. As the flows moves downstream the heat transfer decays until it settles at an approximate value at a location of about 10 hole diameters downstream. As the freestream turbulence intensity is increased the heat transfer coefficient is increased for all cases, and this effect is particularly noticeable compared to the differences between blowing ratios at a given turbulence intensity.

The span-averaged net heat flux reduction (NHFR) is presented in Figure 59 for constant turbulence intensity and varying blowing ratio and in Figure 60 at constant blowing ratio and varying freestream turbulence intensity. The locations of the lowest NHFR are the locations where the heat transfer is highest, specifically at the trailing edge of the coolant hole at a non-dimensional distance (x/D) of 2 . The best overall performing case based on the NHFR is the case when the blowing ratio is 1.5 and the freestream turbulence intensity is $1 \%$. The blowing ratio of 1.5 is the best performer when considering the NHFR for turbulence intensities of 1 and $7.5 \%$, but for the $11.7 \%$ freestream turbulence intensity cases, a blowing ratio of 1.0 was found to be the optimum blowing ratio. The peak for all cases appears to occur between 5-7 hole diameters downstream and a minor secondary peak occurs at approximately 20 hole diameters downstream for the turbulence intensity cases of 7.5 and $11.7 \%$. 


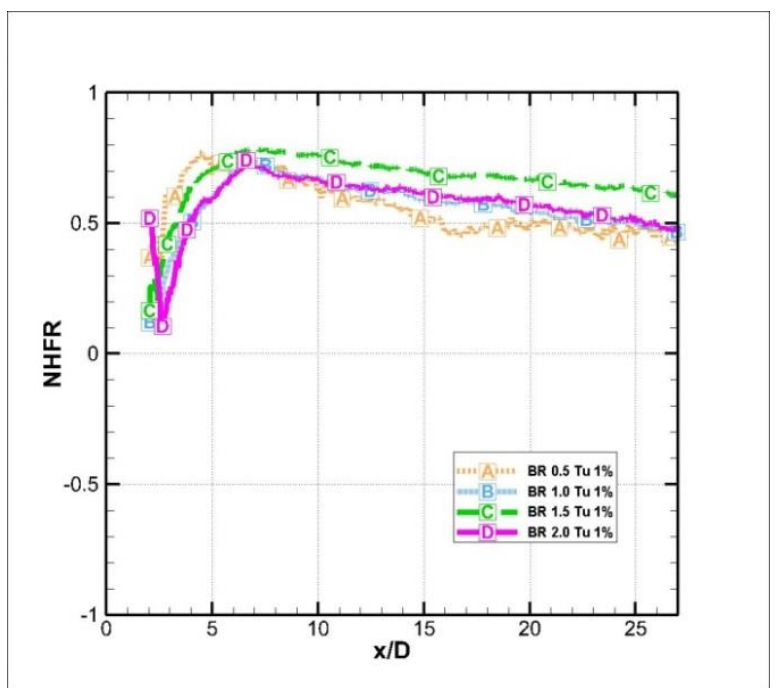

(a)

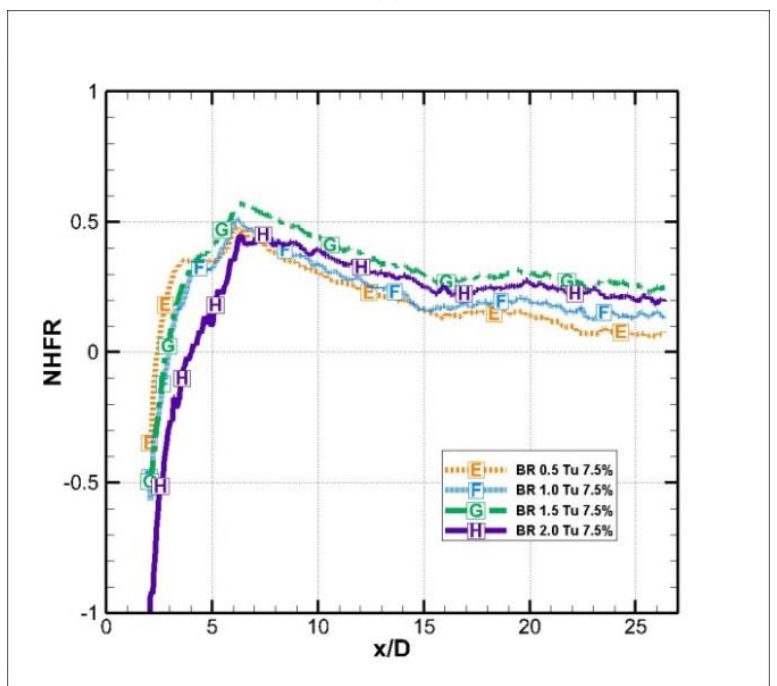

(b)

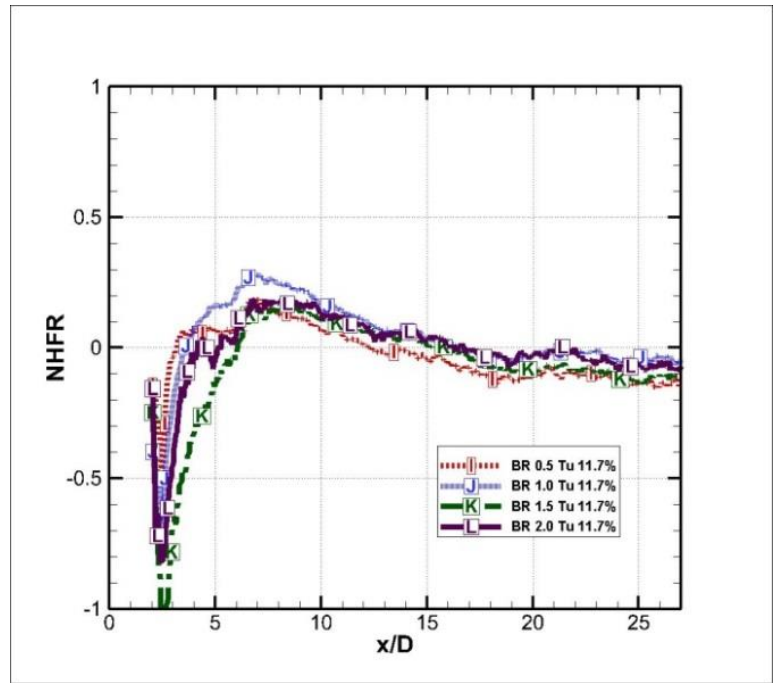

(c)

Figure 59: Span-Averaged NHFR at Varying Blowing Ratio and Constant Turbulence 


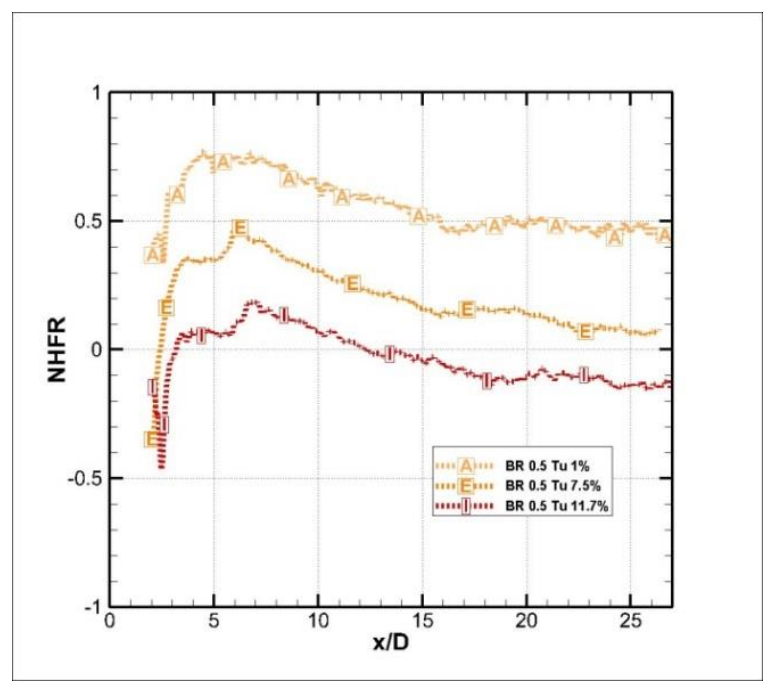

(a)

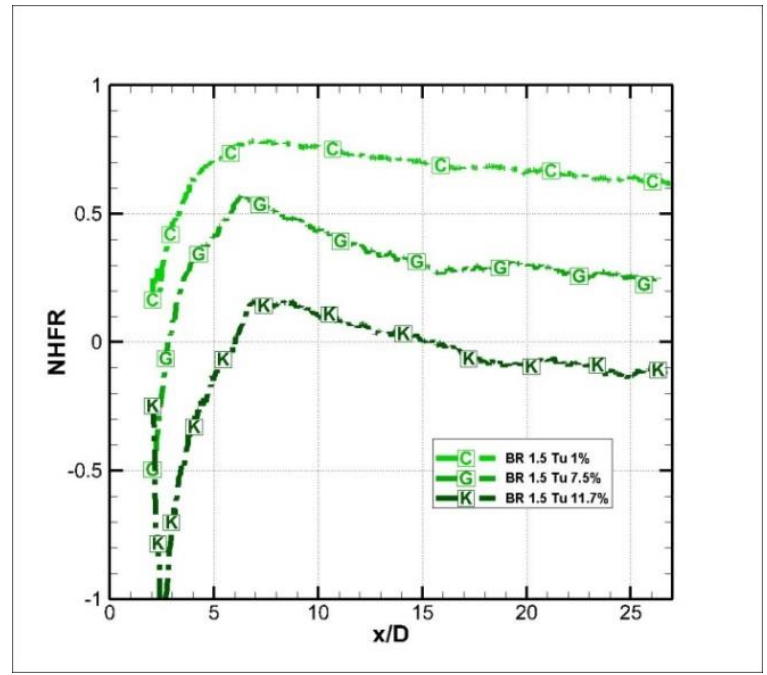

(c)

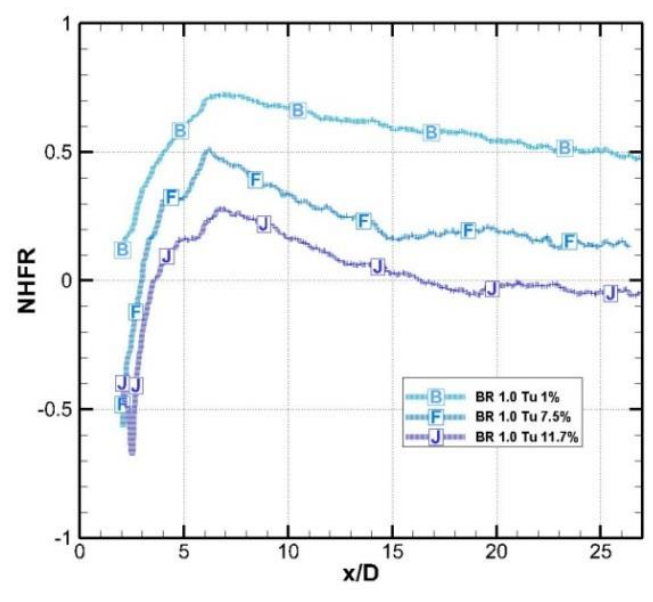

(b)

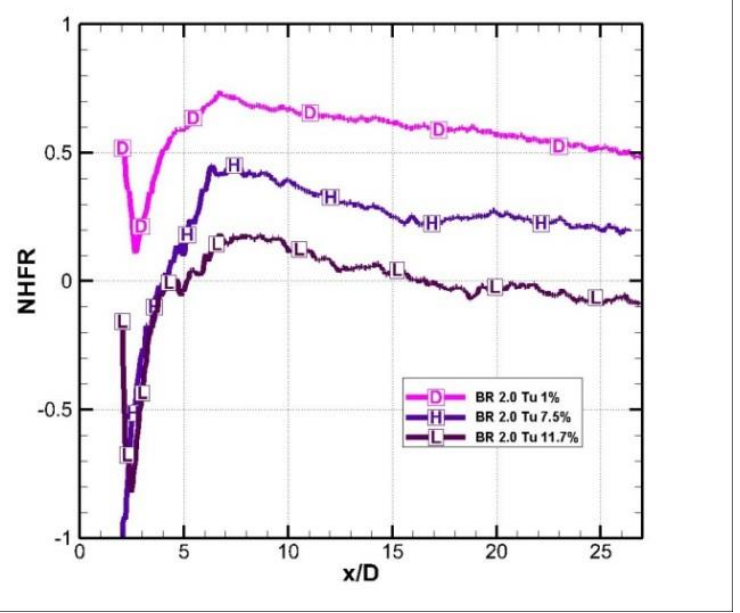

(d)

Figure 60: Span-averaged NHFR for Varying Turbulence Intensity

\subsubsection{ANTI-VORTEX HOLE CENTERLINE HEAT TRANSFER COEFFICIENT}

The non-dimensional centerline convective heat transfer coefficient is presented in

Figure 61 for constant freestream turbulence intensity with varying blowing ratio and in

Figure 62 for a constant blowing ratio and varying freestream turbulence intensity. 


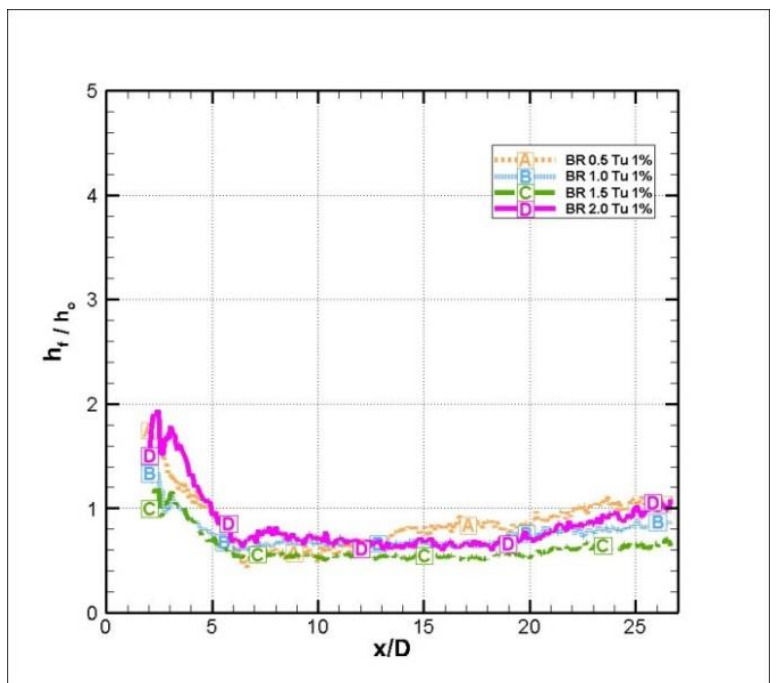

(a)

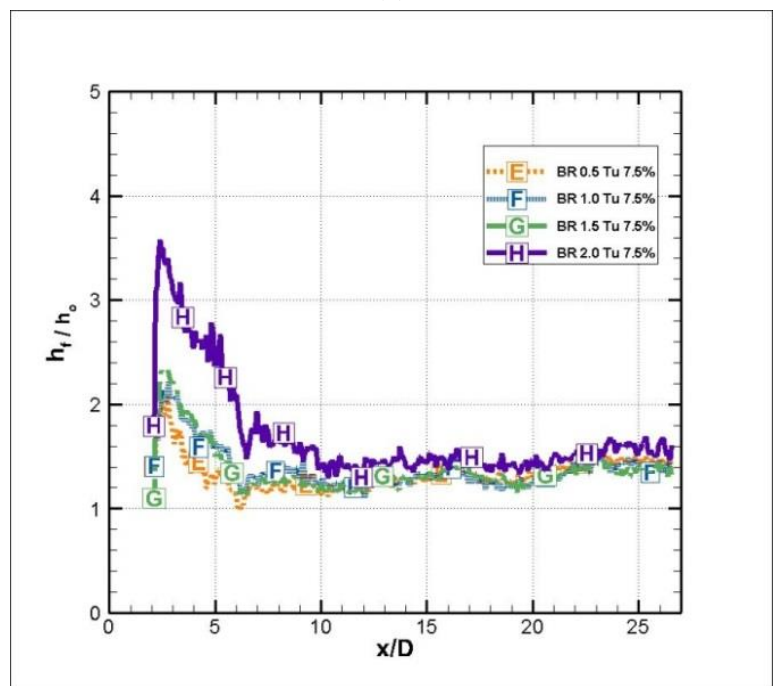

(b)

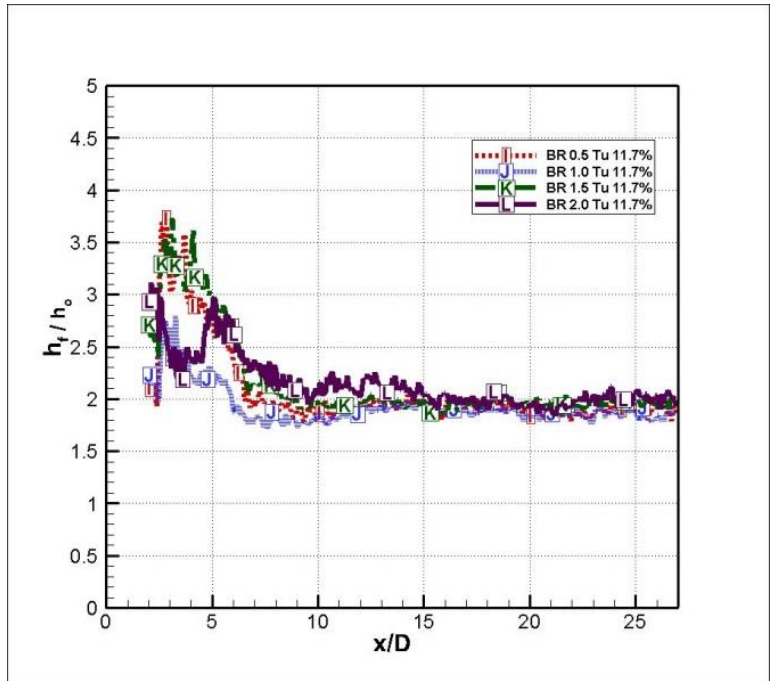

(c)

Figure 61: Centerline Dimensionless Heat Transfer Coefficient at Varying Blowing Ratio 


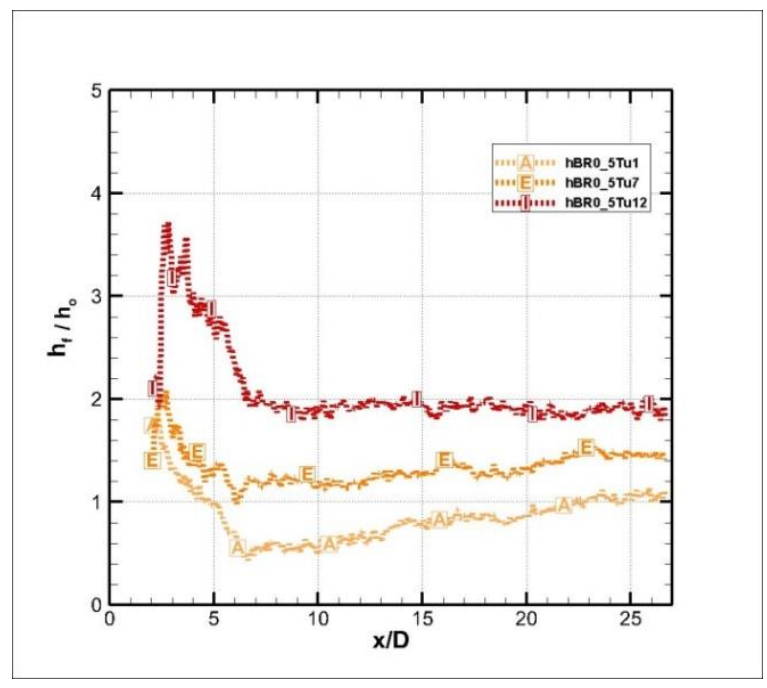

(a)

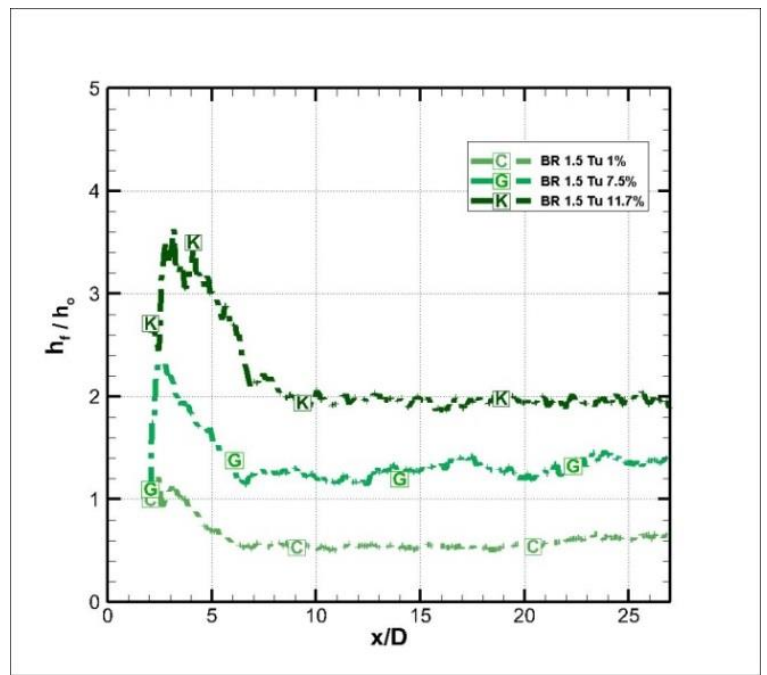

(c)

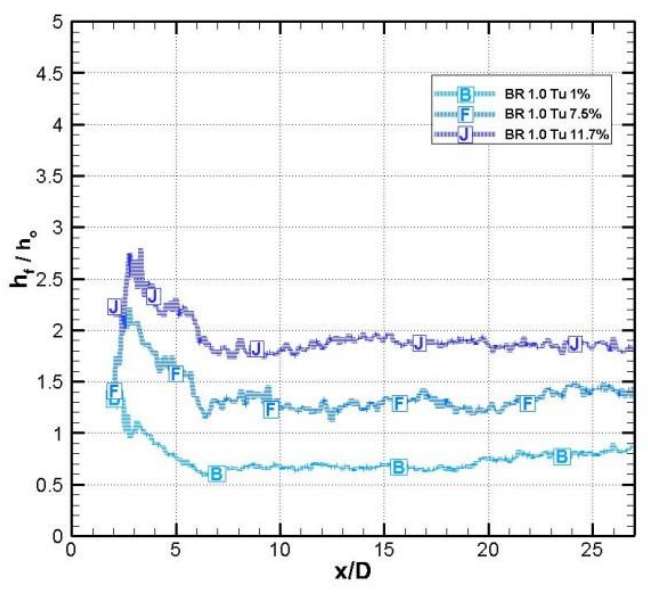

(b)

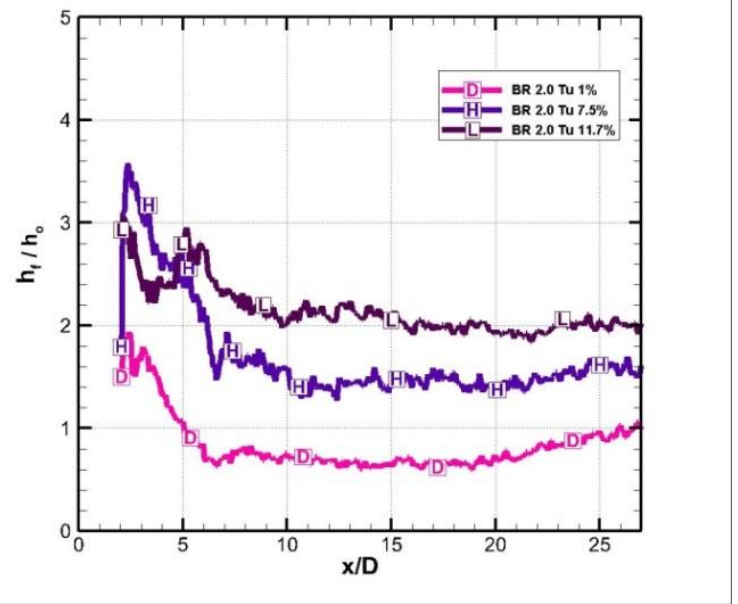

(d)

Figure 62: Centerline Dimensionless Heat Transfer Coefficient for Varying Turbulence Intensity

As the blowing ratio is increased the heat transfer is also increased for all freestream turbulence intensities. As the freestream turbulence intensity is increased the heat transfer coefficient is increased for all cases, and again this trend is particularly noticeable compared to the differences between blowing ratios at a given turbulence intensity. Similarly to the span-averaged heat transfer coefficient, the heat transfer increases with an increase in blowing ratio and is highest in the first 7 hole diameters downstream. 


\subsubsection{ANTI-VORTEX HOLE AREA-AVERAGED HEAT TRANSFER COEFFICIENT}

The area-averaged non-dimensional heat transfer coefficient is beneficial because general ideas about the heat transfer coefficient may be presented to show how varying blowing ratio and freestream turbulence intensity can change the overall amount of heat transfer. Table 7 presents the area-averaged convective heat transfer coefficient, and as expected the heat transfer coefficient is increased in the presence of increased turbulence. The interesting trend to notice is that for the two lower turbulence cases the heat transfer is lowest for a blowing ratio of 1.5 , while at the highest freestream turbulence intensity, the lowest heat transfer occurred at a blowing ratio of 1.0.

Table 7: Area-averaged Dimensionless Heat Transfer Coefficient

\begin{tabular}{|c|c|c|c|}
\hline Case & Tu 1\% & Tu 7.5\% & Tu 11.7\% \\
\hline BR 0.5 & 0.929 & 1.5057 & 2.1033 \\
\hline BR 1.0 & 0.8203 & 1.4813 & 1.9641 \\
\hline BR 1.5 & 0.6315 & 1.4558 & 2.1752 \\
\hline BR 2.0 & 0.8856 & 1.8214 & 2.172 \\
\hline
\end{tabular}




\section{CHAPTER 5: SUMMARY AND CONCLUSIONS}

\subsection{CONCLUSIONS}

A low speed, low temperature wind tunnel facility was designed and constructed by the author to non-dimensionally investigate film cooling effectiveness and heat transfer through a transient infrared thermography technique. Before benchmarking could be performed, the tunnel was fully characterized including design, testing, and evaluation of two freestream passive turbulence grids. Comparing the results of the $30^{\circ}$ inclined straight hole geometry with those of previous experimental investigations showed successful benchmarking of the facility, allowing for the main investigation of an anti-vortex hole geometry at blowing ratios of $0.5,1.0,1.5$, and 2.0 for turbulence intensities of $1,7.5$, and $11.7 \%$.

This facility is the first to experimentally investigate how higher freestream turbulence intensity effects the performance of an anti-vortex hole (AVH) geometry. The primary concern with the geometry is the influence elevated freestream turbulence intensity will have on the secondary holes effectiveness of mitigating the counter rotating vortex (CRV) pair of the main coolant hole. The AVH design used in this study had previously been investigated by Dhungel et al. [13], Heidmann et al. [11, 12], Hunley et al. [53], and Repko et al. [36, 37] and showed that there was a considerable improvement of film cooling effectiveness over the straight hole geometry. Hunley et al. [53] and Repko et al. [36, 37] studied the effect of turbulence intensities of 5, 10, and $20 \%$ for a blowing ratio of 2.0 and found that elevated levels of freestream turbulence predicted improvements in the spanaveraged film cooling effectiveness. The highest turbulence case was found to be the best performer when looking at the span-averaged film cooling effectiveness, which indicates 
that the CRV pairs from the secondary holes are not interfered with by the freestream turbulence. This prediction is contradictory to the current experimental investigation. In this study it was found that the best performing case based on the area-averaged film cooling effectiveness was the blowing ratio of 2.0 and turbulence intensity of $7.5 \%$ case. At this intermediate turbulence intensity the coolant jets are still diffused laterally and the secondary holes appear to be much more effective than in the $11.7 \%$ turbulence cases. The $11.7 \%$ cases, although outperformed by the $7.5 \%$ cases, still performed better than the $1 \%$ cases. This shows that elevated freestream turbulence intensity increases the film cooling effectiveness of the $\mathrm{AVH}$, but the $11.7 \%$ cases experienced a lower film cooling effectiveness than the $7.5 \%$ cases indicating that freestream turbulence intensities greater than $11.7 \%$ could cause performance issues. The highest local film cooling effectiveness occurs directly at the trailing edge of the coolant hole and again 5 cooling hole diameters downstream. It can be ascertained from this study that at high blowing ratios, which are more realistic to engine conditions, the film cooling effectiveness both span-averaged and on the centerline is enhanced by high freestream turbulence.

The convective heat transfer coefficient is increased when the freestream turbulence intensity is increased and also when the blowing ratio is increased. The best overall performing case based off of the NHFR is the case when the blowing ratio is 1.5 and the freestream turbulence intensity is $1 \%$. The blowing ratio of 1.5 is the best performer when considering the NHFR for turbulence intensities of 1 and $7.5 \%$, but for the freestream turbulence intensity cases, a blowing ratio of 1.0 was found to be the optimum blowing ratio. 
The AVH design studied is practical in its approach and could certainly be employed in real gas turbine applications. This design could be much easier to manufacture than that of current shaped holes and would allow for less air to be extracted from upstream in the compressor section leading to higher engine efficiencies. Furthermore, the longevity of the components could be increased reducing the required replacement of components and engine downtime. The only issue with the production of the current design would be the manufacture of the smaller secondary holes and as such current research is being performed by LeBlanc et al. [35] to implement secondary holes with a diameter similar to the main hole.

\subsection{FUTURE WORK}

Future investigation methods in the facility may include the use of thermochromatic liquid crystals and pressure sensitive paint. Future IR thermography may be used in conjunction with systems such as Laser Doppler Velocimetry (LDV) or Particle Image Velocimetry (PIV) systems to determine flow characteristics. If higher turbulence intensities are desired to be studied, the passive turbulence grids would have to be replaced by actively blown turbulence grids.

The effect of boundary layer thickness on film cooling effectiveness and convective heat transfer coefficient could be studied in the future with use of the boundary layer suction system. New geometries can also be easily implemented and explored due to the easily interchangeable plate inserts through the use of the three-dimensional printer. Investigations of surface roughness are also a possibility, to study the effects of surface deposition. In addition, other various film cooling investigations can be performed such as 
leading edge models for showerhead cooling, combustor liner cooling, and contoured endwall cooling. 


\section{REFERENCES}

[1] E. B. Inc. (2014, Sep. 29). Gas-Turbine-Engine (Encyclopedia Britannica Online Academic Edition. ed.). Available:

http://www.britannica.com/EBchecked/topic/226481/gas-turbine-engine

[2] Giampaolo, A., Gas Turbine Handbook Principles and Practices Third Edition. Lilburn, GA: The Fairmont Press, 2006.

[3] Suplee, H. H., The Gas Turbine: Progress in the Design and Construction of Turbines Operated by Gases of Combustion: JB Lippincott Company, 1910.

[4] Hill, P. G. and Peterson, C. R., "Mechanics and Thermodynamics of Propulsion," Reading, MA, Addison-Wesley Publishing Co., 1992, 764 p., vol. 1, 1992.

[5] Hunt, R. J., "The History of the Industrial Gas Turbine (Part 1 The First Fifty Years 1940-1990)," IDGTE, January 2011.

[6] Benson, T., (2014, October 20, 2014). Ideal Brayton Cycle. Available: http://www.grc.nasa.gov/WWW/k-12/airplane/brayton.html

[7] N. Mikalac. (2014, Sep. 29). Power Generation. Available: http://www.mikalac.com/tech/pow/power.html

[8] C. F. P. L. A. R. Group. (2014, Sep. 29). LES of Multiphase Reacting Flows in GasTurbine Combustor. Available: http://web.engr.oregonstate.edu/ sva/gt.html

[9] Han, J. C. and Huh, M., "Recent Studies in Turbine Blade Internal Cooling," presented at the Proceedings of the International Symposium on Heat Transfer in Gas Turbine Systems, Antalya, Turkey, 2009.

[10] Ibarra-Castanedo, C., (Nov. 30, 2007, Sep. 29). Infrared Vision. Available: http://upload.wikimedia.org/wikipedia/commons/6/64/Infrared spectrum.gif

[11] Heidmann, J.D. and Ekkad, S.V., "A Novel Anti-Vortex Turbine Film Cooling Hole Concept," presented at the ASME Turbo Expo, Montreal, Canada, 2007.

[12] Heidmann, J.D., "A Numerical Study of Anti-Vortex Film Cooling Designs at High Blowing Ratio," presented at the ASME Turbo Expo, Berlin, Germany, 2008.

[13] Dhungel, A., Lu, Y., Phillips, W., Ekkad, S.V., and Heidmann, J.D., "Film Cooling From a Row of Holes Supplemented with Anti Vortex Holes," presented at the ASME Turbo Expo, Montreal, Canada, 2007.

[14] Hussein, M.A-M., Mekhail, T.A., Ayad, S.S., and El-Shazly, K.M., "An Ivestigation of the Effect Of Anti-Vortex Film Cooling On a Flat Plate." presented at Tenth International Congress of Fluid Dynamics, Red Sea, Egypt, 2010.

[15] Van Fossen, G.J. and Bunker, R.S., "Augmentation of Stagnation Region Heat Transfer Due to Turbulence from an Advanced Dual-Annular Combustor," presented at the ASME Turbo Expo 2002, Amsterdam, The Netherlands, 2002.

[16] Cumpsty, N., Jet Propulsion. New York, NY, USA: Cambridge University Press, 2003.

[17] Lakshminarayana, B., "Fluid dynamics and heat transfer of turbomachinery, 1996," ed: John Wiley \& Sons, Inc., New York, USA. 
[18] Kadotani, K. and Goldstein, R., "On The Nature of Jets Entering A Turbulent Flow: Part A-Jet-Mainstream Interaction," Journal of Engineering for Gas Turbines and Power, vol. 101, pp. 459-465, 1979.

[19] Kadotani, K. and Goldstein, R. "On the Nature of Jets Entering A Turbulent Flow: Part B-Film Cooling Performance," Journal of Engineering for Gas Turbines and Power, vol. 101, pp. 466-470, 1979.

[20] Mehendale, A.B. and Han, J.C., "Flat Plate Film Cooling With Steam Injection Through One Row and Two Rows of Inclined Holes," ASME Journal of Turbomachinery, vol. 108, pp. 595-607, 1986.

[21] Haven, B., Yamagata, D.L., Kurosaka, M., Yamawaki, S., and Maya, T., "AntiKidney Pair of Vortices in Shaped Holes and Their Influence on Film Cooling Effectiveness," 1997.

[22] Kohli, A., Bunker, D.G., "Adiabatic Effectiveness, Thermal Fields, and Velocity Fields for Film Cooling With Large Angle Injection," ASME Journal of Turbomachinery, vol. 119, pp. 352-358, April 1997.

[23] Bons, J. P., MacArthur, C. D., and Rivir, R., "The Effect of High Freestream Turbulence on Film Cooling Effectiveness," presented at the ASME Turbo Expo, The Hague, Netherlands, 1994.

[24] Lawson, S.A. and Thole, K. A., "The Effects of Simulated Particle Deposition on Film Cooling," presented at the ASME Turbo Expo, Orlando, FL, 2009.

[25] Schmidt, D. L. and Sen, B., "Film Cooling With Compound Angle Holes: Adiabatic Effectiveness," Journal of Turbomachinery, vol. 118, pp. 807-813, 1996.

[26] Ekkad, S.V., Zapata, D., Han, J.C., "Heat Transfer Coefficients Over a Flat Surface with Air $\mathrm{CO}_{2}$ Injection Through Compound Angle Holes Using a Transient Liquid Crystal Image Method," ASME Journal of Turbomachinery, vol. 119, pp. 580-586, April 1997.

[27] Bunker, R.S., "A Review of Shaped Hole Turbine Film-Cooling Technology," Journal of Heat Transfer, vol. 127, pp. 441-453, 2005.

[28] Gritsch, M., Schulz, A., Wittig, S., "Adiabatic Wall Effectiveness Measurements of Film-Cooling Holes With Expanded Exits," ASME Journal of Turbomachinery, vol. 120, pp. 549-556, July 1998.

[29] Wright, L. M., McClain, S. T., Clemenson, M. D., "Effect of Density Ratio on Flat Plate Film Cooling With Shaped Holes Using PSP," ASME Journal of Turbomachinery, vol. 133, October 2011.

[30] Saumweber, C., Schultz, A., Wittig, S., "Free-Stream Turbulence Effects on Film Cooling With Shaped Holes," ASME Journal of Turbomachinery, vol. 125, pp. 6573.

[31] Takeishi, K., Komiyama, M., Oda, Y., Egawa, Y., "Aerothermal Investigations on Mixing Flow Field of Film Cooling With Swirling Coolant Flow," ASME Journal of Turbomachinery, vol. 136, May 2014.

[32] Lu, Y., Nasir, H., Ekkad, S.V., "Film Cooling From a Row of Holes Embedded in Transverse Slots," presented at the ASME Turbo Expo, Reno-Tahoe, Nevada, 2005.

[33] Barigozzi, G., Franchini, G., Perdichizzi, A., "The Effect of an Upstream Ramp on Cylindrical and Fan-Shaped Hole Film Cooling-Part II: Adiabatic Effectiveness Results," presented at the ASME Turbo Expo, Montreal, Canada, 2007. 
[34] Ely, M.J, and Jubran, B.A., "A Numerical Study on Increasing Film Cooling Effectiveness Through the Use of Sister Holes," in ASME Gas Turbo Expo, 2008.

[35] LeBlanc, C., Narzary, D.P., Ekkad, S.V., "Film-Cooling Performance of Antivortex Hole on a Flat Plate," ASME Journal of Turbomachinery, vol. 135, November 2013.

[36] Repko, T.W., Nix, A.C., and J. D. Heidmann, "A Parametric Numerical Study of the Effects of Freestream Turbulence Intensity and Length Scale on Anti-Vortex Film Cooling Design at High Blowing Ratio," in ASME 2013 Summer Heat Transfer Conference, Minneapolis, MN, USA, 2013.

[37] Repko, T.W., "Numerical Investigation of the Influence of Elevated Turbulence Levels on the Cooling Effectiveness of an Anti-Vortex Hole Geometry," Master of Science in Aerospace Engineering, Mechanical and Aerospace Engineering, West Virginia University, 2014.

[38] Bradshaw, P. and Mehta, R. (2014,Sep. 29 ). Wind Tunnel Design. Available: http://navier.stanford.edu/bradshaw/tunnel/index.html

[39] Baines, W.D. and Peterson, E.G., "An Investigation of Flow Through Screens," Iowa City: ASME,1951. pp. 467-480.

[40] Radomsky, R.W. and Thole, K.A., "Detailed Boundary Layer Measurements on a Turbine Stator Vane at Elevated Freestream Turbulence Levels," ASME Journal of Turbomachinery, vol. 124, pp. 107-118, January 2002.

[41] Jenkins, S., Varadarajan, K., and Bogard, D.G., "The effects of high mainstream turbulence and turbine vane film cooling on the dispersion of a simulated hot streak," Journal of turbomachinery, vol. 126, pp. 203-211, 2004.

[42] University of Michigan Dearborn, "Meriam Laminar Flow Element Instruction Manual," ed. Cleveland, Ohio: The Meriam Instrument Co., 1961.

[43] Dantec Dynamics. (2014,Sep. 29). Constant Temperature Anemometry. Available: https://www.dantecdynamics.com/hot-wire-probes

[44] F. Systems, "FLIR A655sc Specifications Sheet," ed: FLIR, 2014, pp. 1-2.

[45] Nix, A.C., "Effects of High Intensity, Large-Scale Freestream Combustor

Turbulence on Heat Transfer in Transonic Turbine Blades," Ph. D thesis, Virginia Polytechnic Institute and State University, 2003.

[46] Taylor, G.I., "The spectrum of turbulence," Proceedings of the Royal Society of London. Series A-Mathematical and Physical Sciences, vol. 164, pp. 476-490, 1938.

[47] Ekkad, S.V., Ou, S., Rivir, R.B., "A Transient Infrared Themography Method for Simultaneuous Film Cooling Effectiveness and Heat Transfer Coefficient Measurements From a Single Test," ASME Journal of Turbomachinery, vol. 126, pp. 597-603, October 2004.

[48] Vendula, R.J. and Metzger, D.E., "A Method for the Simultaneous Determination of Local Effectiveness and Heat Transfer Distributions in Three-Temperature Convection Situations," presented at the International Gas Turbine and Aeroengine Congress and Exposition, Orlando, FL 1991.

[49] Lu, Y., "Effect of Hole Configurations on Film Cooling from Cylindrical Inclined Holes for the Application to Gas Turbine Blades," Ph. D thesis, Virginia Polytechnic Institute and State University, 2007. 
[50] Moffat, R.J., "Describing the uncertainties in experimental results," Experimental Thermal and Fluid Science, vol. 1, pp. 3-17, 1988.

[51] Russin, R.A., Alfred, D., Wright, L.M., "Measurement of Detailed Heat Transfer Coefficient and Film Cooling Effectiveness Distributions using PSP and TSP," presented at the ASME Turbo Expo, Orlando, Florida, 2009.

[52] Sen, B., Schmidt, D.L., and Bogard, D.G., "Film cooling with compound angle holes: heat transfer," Journal of Turbomachinery, vol. 118, pp. 800-806, 1996.

[53] Hunley, B. K., Nix, A.C., and Heidmann, J.D., "A Preliminary Numerical Study on the Effect of High Freestream Turbulence on Anti-Vortex Film Cooling Design at High Blowing Ratio," presented at the ASME Turbo Expo, Glasgow, Scotland, 2010.

[54] Mathieu, J. and Scott, J., An introduction to turbulent flow: Cambridge University Press, 2000. 


\section{APPENDIX A: TURBULENCE}

It is important to note that laminar flows are much easier to study than turbulent flows, but the fact of the matter is that in the majority of real world applications the flow is turbulent. Therefore, it is out of necessity that turbulence must be studied to understand what is truly occurring or to better predict what is occurring. The freestream flow in gas turbine engines is a highly turbulent environment due to the flow through the compressor, fuel swirlers and transient combustion processes in the combustor, and coolant air introduction as well as from the complex flow patterns the working fluid undergoes which results in enhanced heat transfer rates. Turbulence arises generally from instability of a laminar base flow as the Reynolds number is increased and is defined as:

$$
\operatorname{Re}=\frac{\rho U L}{\mu}=\frac{U L}{v}, \text { where } v=\frac{\mu}{\rho}
$$

With manipulation of equation (A-1), Reynolds number can be shown mathematically as the ratio of inertial forces over the viscous forces as follows:

$$
\frac{\rho U L}{\mu} * \frac{U L}{U L}=\frac{\rho U^{2} L^{2}}{\mu \frac{U}{L} L^{2}} \propto \frac{q_{\infty} S}{\tau_{w} S}=\frac{F_{\text {inertial }}}{F_{\text {viscous }}}
$$

From the equation (A-2) it can be seen that, as the Reynolds number becomes larger, the viscous forces eventually are unable to dampen disturbances caused by the inertial forces. Due to the intricacies of turbulence a short definition is not possible. Presented in the following sections will be 11 vital characteristics that Mathieu and Scott [54] attempt to use to better define and understand turbulence.

1. Turbulence is/appears to be random or chaotic in both the time and space domains. Hotwire anemometry is an easy way to observe this characteristic by taking the 
signal and plotting it versus time to show the "fuzziness" or the randomness which occurs. These plots are visible in section 3.1.

2. Turbulence consists of various length and time scales. Taking the plot described in characteristic 1, the largest eddy size is realized and is also known as the integral length scale $(\Lambda)$. Zooming in on a plot of a hotwire signal will depict even smaller fluctuations or "fuzz" which shows the existence of smaller length scale turbulence. The smallest eddy size is known as the Kolmogorov length scale or dissipation length scale $\left(\eta_{\mathrm{k}}\right)$.

3. Turbulence is a continuum phenomenon. The smallest scales are dictated by viscosity and usually are many orders larger than the mean free path. Therefore, turbulence can be governed by the Navier-Stokes equation and other continuum approximations. The incompressible Navier-Stokes equations can be seen mathematically in equations (A-3) and (A-4).

$$
\begin{gathered}
\frac{\partial \mathrm{U}_{\mathrm{i}}}{\partial \mathrm{t}}+\mathrm{U}_{\mathrm{j}} \frac{\partial \mathrm{U}_{\mathrm{i}}}{\partial \mathrm{x}_{\mathrm{j}}}=-\frac{1}{\rho} \frac{\partial \mathrm{P}}{\partial \mathrm{x}_{\mathrm{i}}}+v \frac{\partial^{2} \mathrm{U}_{\mathrm{i}}}{\partial \mathrm{x}_{\mathrm{j}} \partial \mathrm{x}_{\mathrm{j}}} \\
\frac{\partial \mathrm{U}_{\mathrm{i}}}{\partial \mathrm{x}_{\mathrm{i}}}=0
\end{gathered}
$$

where the first term in equation (A-3) is the local velocity term, the second term is the convective acceleration, the third term is the pressure gradient, and the last term is the viscous term.

4. Turbulence contains small-scale, random vorticity. This vorticity is intense and varies in the time and space domains.

5. Turbulence is three-dimensional. When analyzing turbulence in two dimensions vortex stretching does not exist and the dissipation scales are not realized. This 
suggests that the smaller scale eddies all merge to form larger scale eddies which is the inverse of what happens in reality.

6. Turbulence occurs at high Reynolds number.

7. Turbulence dissipates energy at the Kolmogorov length scale in the form of heat in a cascade effect starting from the integral length scale eddies.

8. Turbulence has large eddy scales that become independent of Reynolds number for very large Reynolds numbers. At high Reynolds number the dynamics of the large scales become essentially inviscid resulting in being practically insensitive to fluctuations in Reynolds number.

9. Turbulence has small scale eddies (Kolmogorov length scale) that tend towards being isotropic, homogeneous, and universal as Reynolds number is increased.

10. Turbulence tends to be intermittent.

11. Turbulence is a non-linear phenomenon. Mathematically it can be shown as due to the convective acceleration term in the incompressible Navier Stokes equations. 